\title{
Contributions to the evidence base for reducing the impact of influenza in primary care
}

Citation for published version (APA):

Verhees, R. A. F. (2021). Contributions to the evidence base for reducing the impact of influenza in primary care. [Doctoral Thesis, Maastricht University]. Ridderprint. https://doi.org/10.26481/dis.20211126rv

Document status and date:

Published: 01/01/2021

DOI:

10.26481/dis.20211126rv

Document Version:

Publisher's PDF, also known as Version of record

\section{Please check the document version of this publication:}

- A submitted manuscript is the version of the article upon submission and before peer-review. There can be important differences between the submitted version and the official published version of record. People interested in the research are advised to contact the author for the final version of the publication, or visit the DOI to the publisher's website.

- The final author version and the galley proof are versions of the publication after peer review.

- The final published version features the final layout of the paper including the volume, issue and page numbers.

Link to publication

\footnotetext{
General rights rights.

- You may freely distribute the URL identifying the publication in the public portal. please follow below link for the End User Agreement:

www.umlib.nl/taverne-license

Take down policy

If you believe that this document breaches copyright please contact us at:

repository@maastrichtuniversity.nl

providing details and we will investigate your claim.
}

Copyright and moral rights for the publications made accessible in the public portal are retained by the authors and/or other copyright owners and it is a condition of accessing publications that users recognise and abide by the legal requirements associated with these

- Users may download and print one copy of any publication from the public portal for the purpose of private study or research.

- You may not further distribute the material or use it for any profit-making activity or commercial gain

If the publication is distributed under the terms of Article $25 \mathrm{fa}$ of the Dutch Copyright Act, indicated by the "Taverne" license above, 


\section{CONTRIBUTIONS TO THE EVIDENCE BASE FOR REDUCING THE IMPACT OF INFLUENZA IN PRIMARY CARE}

Ruud Andreas Fritz Verhees 
ISBN: 978-94-6416-742-9

Cover design and layout: (C) evelienjagtman.com

Printing: Ridderprint

The research presented in this thesis was funded by a Kootstra Talent Fellowship from Maastricht University Medical Center, Bavo Stichting and CAPHRI Care and Public Health Research Institute.

Printing of this thesis was financially supported by Maastricht University, $\mathrm{SBOH}$ and Stichting Gezondheidscentra Eindhoven (SGE).

Copyright (c) 2021 R.A.F. Verhees

All rights reserved. No parts of this thesis may be reproduced in any form without written permission of the author or copyright-owning journals for previously published chapters. 


\title{
CONTRIBUTIONS TO THE EVIDENCE BASE FOR REDUCING THE IMPACT OF INFLUENZA IN PRIMARY CARE
}

\author{
Proefschrift \\ ter verkrijging van de graad van doctor \\ aan de Universiteit Maastricht, op gezag van de Rector Magnificus, \\ Prof. dr. Rianne M. Letschert volgens het besluit \\ van het College van Decanen, in het openbaar te verdedigen \\ op vrijdag 26 november 2021 om 12.00 uur
}

door

Ruud Andreas Fritz Verhees

geboren op 22 november 1988 te Geldrop 


\section{Promotores}

Prof. dr. G.J. Dinant

Prof. dr. J.A. Knottnerus

\section{Copromotor}

Dr. C.T. Thijs

\section{Beoordelingscommissie}

Prof. dr. C.J.P.A. Hoebe (voorzitter)

Prof. dr. R.A.M. Fouchier (Erasmus Medisch Centrum, Rotterdam)

Dr. W. Opstelten (Nederlands Tijdschrift voor Geneeskunde)

Prof. dr. T. van der Weijden 


\section{CONTENT}

Chapter 1 General introduction 7

Chapter 2 Influenza vaccination in the elderly: is a trial on mortality ethically acceptable?

Vaccine 2018;36:2991-7

Ned Tijdschr Geneeskd. 2018;162:D3177

Chapter 3 Influenza vaccination among Dutch general practitioners and their attitude towards influenza vaccination in the elderly Hum Vaccin Immunother. 2020;16:2709-18

Chapter 4 Influenza vaccination in the elderly: mortality after 25 years followup of a trial

PloS One 2019;23:e0216983

Huisarts Wet 2020;63:16-9

Chapter 5 Antibody response following influenza vaccination in the elderly and long-term survival: a follow-up study based on a randomized controlled trial

Submitted

Chapter 6 Influenza point-of-care tests in the general practice and emergency department: analytical accuracy and added value Ned Tijdschr Geneeskd. 2019;163:D3806

Chapter 7 Summary and general discussion

Addendum Impact paragraph 155

Samenvatting 165

Curriculum vitae

173

List of publications 177

Dankwoord 181 

Chapter 1

General introduction 



\section{GENERAL INTRODUCTION}

Annually about 5-10\% of the population gets infected by influenza. This is not always harmless and can be associated with serious complications, especially in the elderly and those with certain medical conditions. In the elderly, chances of getting influenza can be reduced by $50 \%$ by receiving influenza vaccination. ${ }^{1}$ For the time being, vaccination is the most effective strategy to prevent influenza. In the Netherlands, the influenza vaccination programme is carried out by the general practitioner (GP). In addition to vaccinating those at risk for complications, vaccination of healthcare workers (HCWs) is also considered an important measure in reducing the impact of influenza, because it would reduce the risk of absenteeism and transmission to those at risk for complications. However, vaccination coverage among both the elderly and HCWs is well below the targeted $75 \%{ }^{2}$ and $90 \%$ respectively. ${ }^{3}$

If not dealt with by proper prevention and control, an influenza epidemic can lead to a sudden increase in elderly care needs, resulting in capacity problems in, and obstruction of the care pathway. In this thesis, I evaluate different perspectives that could contribute to reducing the burden of disease caused by influenza, such as: long-term effects of vaccination, attitude of GPs regarding influenza vaccination, point-of-care testing for influenza, methodological and ethical aspects involved in conducting influenza vaccination studies and the relation between immune response following influenza vaccination and long-term mortality. In the introduction, I first explain why it is important to prevent influenza among the elderly and what might explain for their low influenza vaccination coverage rate. Then, I discuss some options for reducing the impact of influenza; both in the field of prevention and diagnostics.

\section{Preventing the flu, why would you?}

An influenza infection is characterized by the triad of (1) acute onset, (2) cough, sore throat, cold symptoms and (3) fever, arthralgia, headache, chills, malaise, fatigue and myalgia. ${ }^{4}$ However, the symptoms may present considerably less characteristic, making it difficult to distinguish influenza from other respiratory infections based on only history taking and physical examination. ${ }^{4,5}$ In addition to these unpleasant symptoms, influenza also gives an increased risk of serious complications. Examples are pulmonary complications such as influenza pneumonia, secondary bacterial pneumonia and exacerbations of pre-existent lung disease (e.g. chronic obstructive pulmonary disease or asthma). Less known are extra-pulmonary complications such as cardiac and cerebrovascular events, myocarditis and rhabdomyolysis. ${ }^{6}$

Because not all influenza cases are registered, precise statistics about the risk of complications due to influenza are not known. Complications can occur in all elderly people, but especially in those with a (known) medical indication for influenza vaccination such as cardiovascular or pulmonary disease, renal disease or diabetes mellitus, the risk for complications seems higher than in healthy elderly. For example, a study predominantly conducted in elderly known with cardiovascular disease, showed that after undergoing a proven influenza infection, one in eight 
patients show abnormal blood tests indicative for having gone through a myocardial infarction after the infection. ${ }^{6}$ This risk is significantly smaller when picking up other respiratory tract infections. ${ }^{8}$ The chance of hospitalization due to influenza also appears to depend on the presence of comorbidities and ranges from $0-8.8 \%$. In the Netherlands, it is estimated that between 2,000 and 10,000 patients are admitted annually because of complications due to influenza. ${ }^{9}$ Ultimately, 4 to $17 \%$ of admitted patients end up at an intensive care unit. ${ }^{7}$ The complications from influenza can even lead to death. In the Netherlands, influenza related deaths range from 400 to 9,400 patients annually. In an analysis of influenza morbidity and mortality among subjects over 65 years of age, the influenza case fatality rate was estimated at 0.5 to $3 \% .4,10$

Due to the high incidence, the risk of serious complications and the increasing age of population, the burden of disease caused by influenza is high. ${ }^{11}$ As a result, the workload of doctors and nurses in primary and secondary care increases during the flu seasons, as well as bed occupancy. ${ }^{12,13}$ This can lead to capacity problems and obstruction of the care pathway. Therefore, from both individual and social perspective, it is important to prevent influenza, or to limit the severity and duration of the disease course.

\section{Why is influenza vaccination coverage low among the elderly?}

Although the vaccination coverage for influenza in the Netherlands is relatively high compared to other European countries, among the elderly it has been below the WHO target level of 75\% for years. ${ }^{2}$ In 2019, the previously declining vaccination coverage among the elderly stabilized; $54.8 \%$ compared to 53.8\% in 2018.9.14 By time of writing of this thesis, new statistics on the winter of 2020-2021 in which the SARS-CoV-2 pandemic started, had not yet been published. Among elderly people with a medical indication (about half of all elderly), vaccination coverage is higher than among healthy elderly: 67.6\% and 44.4\% respectively (winter 2019-2020). ${ }^{15}$ Out of the young healthy elderly aged 60 years who receive an invitation for influenza vaccination for the first time, only $22 \%$ gets vaccinated. ${ }^{16}$ About $25 \%$ of the Dutch elderly report a 'general attitude' against influenza vaccination. ${ }^{17}$ Common reasons for the elderly to refuse influenza vaccination are the absence of medical indications and the experience of good health (complacency) ${ }^{18-20}$ or not sharing the perception to be at risk for complications of influenza. ${ }^{21}$ The hazards of influenza are often underestimated. ${ }^{22}$ In addition, lack of trust in vaccination is an important factor in rejecting influenza vaccination. ${ }^{19,} 20$ In a systematic review, lack of confidence (low perceived vaccine effectiveness, higher perceived risk induced by the vaccine) was identified as the most commonly reported barrier for influenza vaccine uptake among the elderly. ${ }^{20}$ Negative perceptions about the effectiveness and safety of the vaccine correlate with lower vaccination coverage among the elderly. ${ }^{18,23,24}$ This underlines the importance of providing adequate information for this target group on health risks following influenza, vaccine effectiveness and vaccine safety. Also, the GP can play an important role in this, given his/her position in the influenza vaccination policy. For instance, a study performed in Switzerland showed that the influenza coverage rate in patients increased significantly when GPs recommended the vaccine to their patients. ${ }^{25}$ 
The effectiveness of influenza vaccination: beliefs and discussion

As previously set out, uncertainty about the effectiveness and safety of influenza vaccination are important negative predictors for influenza vaccine uptake. However, since beliefs about vaccine efficacy may vary from one year to another and between different countries and cultures, it is difficult to express belief in the efficacy of influenza vaccination of the elderly patient as one percentage. A study performed in elderly Americans showed that 'only' 5-11\% of the elderly beliefs influenza vaccination is not effective. ${ }^{23}$ Also according to a systematic review, less than one in five GPs question the effectiveness (2.5-16\%) or safety (7.5-17.5\%) of influenza vaccination when it comes to their personal choice to get vaccinated. ${ }^{26}$ Nevertheless, this topic is strongly debated in the scientific society. ${ }^{27,28}$ When discussing the effectiveness of influenza vaccination, it is relevant to be transparent about the outcome measure in which effectiveness is expressed: for instance clinical protection (does the vaccinated subject show symptoms of influenza), serological protection, hospitalization or death. Before reviewing the effectiveness of influenza vaccination on various endpoints, it should be emphasized that a good antigenic match between the vaccine strain and the actual circulating virus is a precondition for vaccine efficacy. Currently, in spite of worldwide surveillance of new influenza strains and variants and seasonal adaptation of the vaccine composition, ${ }^{29}$ it is difficult to predict which influenza viruses might become dominant circulating strains. For example, it appears that in only four out of eleven (winter) seasons, influenza vaccines were properly matched to the circulating viral strains. ${ }^{30}$

The clinical effectiveness of influenza vaccination in the elderly has been demonstrated by means of two randomized placebo-controlled trials. ${ }^{1,31}$ One of these trials, also the largest and methodologically strongest trial in the field of influenza vaccination, was conducted in 1991/1992 in the Netherlands and showed that influenza vaccination in the elderly can reduce the risk of getting influenza by 50\%. ${ }^{1}$ Based on trial data, the most recent Cochrane meta-analysis comes to similar findings. ${ }^{32}$ In addition, there are many (registration) studies showing that vaccination can induce an adequate (protective) antibody response thus induce so called serological protection. This so-called serological response is an intermediate but reliable predictive measure for clinical protection against influenza. ${ }^{33}$ The available RCTs and registration studies are usually only big enough to provide for evidence on clinical, laboratory proven (by means of polymerase chain reaction (PCR) test or antibody test) or serological protection against influenza, but not for outcome measures such as mortality or hospitalization. However, aim of influenza vaccination is predominantly to reduce complications and/or mortality due to infection. Discussion about the effectiveness of the influenza vaccine largely emanates from the absence of the direct evidence on the later outcome measures. ${ }^{34}$ However, a part of excess winter deaths is clearly related to influenza incidence. Although not proven by direct evidence, it seems plausible that prevention of influenza also prevents death from influenza. Several (observational) studies also show such an effect. However, these studies have important methodological limitations such as bias and confounding, ${ }^{35,36}$ fuelling the discussion regarding the effect of influenza vaccination on mortality. 


\section{How can the impact of influenza further be reduced?}

The preceding shows that influenza is a major societal problem. Underestimation of the hazards of influenza, overestimation of one's perceived health status and lack of transparency about the effectiveness of influenza vaccination seem to play an important role in explaining for the low vaccine coverage rates. In this thesis, I focus on different perspectives that could contribute to reducing the impact of influenza.

\section{Attitude of the GP towards influenza vaccination}

Much research has been conducted evaluating interventions aimed at increasing the vaccination coverage among patients. A systematic review shows that several strategies can be effective, for instance: actively reminding patients of their invitation to get vaccinated, personal invitation by doctor assistants, home visits by healthcare providers, reminding doctors to vaccinate their patients and using informative posters in family practices. ${ }^{37}$ Whereas GPs play a key role in influenza vaccination policy in the Netherlands, unfortunately these interventions are only applied in study contexts and implementation in Dutch general practices is lacking.

We know that the personal vaccination behavior and the attitude of doctors towards influenza vaccination play an important role in the vaccination uptake of patients. ${ }^{38,39}$ Between Dutch GP-practices, large differences in influenza vaccination coverage rates in patients are seen that cannot be explained by differences in the composition of the targeted riskgroups. ${ }^{40}$ However, the attitude of Dutch GPs towards influenza vaccination has not been evaluated in the past ten years. By conducting a survey among GPs, we wanted to gain more insight into GPs' attitudes towards influenza vaccination, as this can offer clues to influence the vaccination behavior of their patients.

Information on the effects of influenza vaccination on mortality in the elderly

Providing accurate information to the elderly about the effects of influenza vaccination also appears to be an effective strategy in increasing the influenza vaccination rate. ${ }^{37}$ Given the discussion about the effectiveness of influenza vaccination in the elderly and the limitations of the available evidence, the question is whether a new large influenza vaccination trial focusing on outcome measures such as hospitalization or mortality should be set up. Such a trial could clarify the relationship between vaccination and mortality among the elderly and contribute to better information provision. At this moment, a comprehensive analysis on the ethical acceptability and methodological feasibility of an influenza trial on mortality has never been published. Such an analysis could help to move the discussion forward and prepare for future evaluation of new influenza vaccines.

Besides the effect of vaccination on seasonal mortality, adequate information about the longterm effects of vaccination is also relevant. In the end, by preventing influenza, the complications caused by influenza that can negatively affect the survival of the elderly in the long term can also be prevented. Prevention of (accumulation) of influenza-related complications together with 
possible residual protection of the vaccine could therefore result in long-term survival benefits. Positive findings with regard to such long-term effects could influence vaccination trust, thus support elderly to decide to accept influenza vaccination or not.

Previous research has shown reasonable to good immune responses following influenza vaccination in the elderly. ${ }^{41,42}$ Responses following influenza vaccination can also be studied in a broader perspective. In addition to evaluating the long-term effects of vaccination on mortality, we also explored associations between the antibody response following influenza vaccination which may partly reflect humoral immune status in a broader sense - and survival.

Rapid diagnostics of influenza virus infection and its added value for clinical practice Finally, in addition to prevention through vaccination, early diagnosis of influenza could also help limiting the impact of influenza. In general practice, influenza is usually diagnosed by history taking and physical examination (influenza is considered a so called 'clinical diagnosis'). However, we do know that by these means, different respiratory tract infections cannot be accurately discriminated. ${ }^{5}$ Rapid identification of influenza is also desirable for emergency care. If it takes long before influenza test results come in, or if one omits influenza diagnostics, this can not only lead to defensive diagnostics and (unnecessary) treatment with antibiotics, but also avoidable hospital referrals, admissions and isolation of patients with influenza-like symptoms. Diagnosing influenza more rapidly may improve patient care, reduce the risk of obstruction of the care pathway during influenza epidemics and reduce healthcare costs and the workload of healthcare providers.

\section{Objective of this dissertation and main research questions}

In this thesis, I examine different perspectives that could contribute to the reduction of the impact of influenza. The principal questions of this thesis are:

- What is the current influenza vaccine coverage rate in general practitioners? How do general practitioners think about influenza vaccination and related topics such as mandatory influenza vaccination for healthcare workers and conducting a new influenza vaccination trial with mortality as an endpoint?

- Which ethical and methodological barriers have to be addressed if one would want to conduct an influenza vaccination trial targeting mortality or hospitalization among the elderly?

- Can a long-term effect of influenza vaccination on mortality in the elderly be supported with direct evidence?

- How do antibody responses following influenza vaccination in the elderly relate to overall survival?

- What role can rapid influenza testing play in reducing the impact of influenza? 


\section{Outline of this dissertation}

In chapter 2 the scientific context of influenza vaccination in the elderly is discussed. We focus on the ethical and scientific challenges of a new placebo-controlled RCT designed to evaluate the efficacy of influenza vaccination in the elderly on mortality, and explore whether or not these challenges can be overcome. Chapter 3 describes the knowledge, attitudes and beliefs of general practitioners towards influenza vaccination in different regions in the Netherlands before and during the 2018/2019 influenza epidemic. Over 1,600 GPs were asked to fill in a questionnaire either before or during the influenza epidemic. Chapter 4 provides the results of a 25 -year follow-up study of an RCT on the efficacy of influenza vaccination at baseline. The aim of this study was to assess the long-term outcome of (singular) influenza vaccination on mortality in the elderly. In chapter 5 we used the same follow-up data of this RCT for a more explorative study that aimed to answer the question whether besides the effect of vaccination as such, the immune response elicited by vaccination might be an indicative marker for immune responsiveness. Therefore, we evaluated the relation between antibody response following influenza vaccination in the elderly and mortality. In chapter 6 we present the results of a narrative review on the analytical accuracy of influenza point-of-care tests used in different settings (i.e. general practice and the emergency department) and its impact on clinically relevant outcome measures such as prescribing antibiotics and antiviral drugs, hospital admissions and length of stay in the emergency department and admission time. The objective of this review was to determine the added value of these point-of-care tests for daily practice in both settings: general practice and emergency department. Finally, in chapter 7, the general discussion, I discuss the main findings described in earlier chapters and place these in a broader context including methodological considerations, implications for clinical practice and recommendations for future research. 


\section{REFERENCES}

1. Govaert TM, Thijs CT, Masurel N, Sprenger MJ, Dinant GJ, Knottnerus JA. The efficacy of influenza vaccination in elderly individuals. A randomized double-blind placebo-controlled trial. Jama. 1994;272:1661-5.

2. Coverage rate influenza vaccination in Europe according to WHO [cited January 2020]. Available from: http://www.euro.who.int/en/health-topics/communicable-diseases/influenza/vaccination/seasonalvaccination-policies-and-coverage-in-the-european-region.

3. Healthy people goal 2020 [cited May 2021]. Available from: https://www.healthypeoplegov/2020/ topics-objectives.

4. Guideliness influenza by RIVM [cited May 2021]. Available from: https://lci.rivm.nl/richtlijnen/ influenza.

5. Govaert TM, Dinant GJ, Aretz K, Knottnerus JA. The predictive value of influenza symptomatology in elderly people. Fam Pract. 1998;15:16-22.

6. Sellers SA, Hagan RS, Hayden FG, Fischer WA. The hidden burden of influenza: A review of the extrapulmonary complications of influenza infection. Influenza Other Respir Viruses. 2017;11:372-93.

7. Mauskopf J, Klesse M, Lee S, Herrera-Taracena G. The burden of influenza complications in different high-risk groups: a targeted literature review. J Med Econ. 2013;16:264-77.

8. Corrales-Medina VF, Musher DM, Wells GA, Chirinos JA, Chen L, Fine MJ. Cardiac complications in patients with community-acquired pneumonia: incidence, timing, risk factors, and association with short-term mortality. Circulation. 2012;125:773-81.

9. Complications following influenza [cited May 2021]. Available from: https://www.rivm.nl/griepgriepprik/feiten-en-cijfers.

10. Denoeud L, Turbelin C, Ansart S, Valleron AJ, Flahault A, Carrat F. Predicting pneumonia and influenza mortality from morbidity data. PLoS One. 2007;2:e464.

11. van Lier A, McDonald SA, Bouwknegt M, et al. Disease Burden of 32 Infectious Diseases in the Netherlands, 2007-2011. PLoS One. 2016;11:e0153106.

12. Sauro A, Barone F, Blasio G, Russo L, Santillo L. Do influenza and acute respiratory infective diseases weigh heavily on general practitioners' daily practice? Eur J Gen Pract. 2006;12:34-6.

13. Beysard N, Yersin B, Meylan P, Hugli O, Carron PN. Impact of the 2014-2015 influenza season on the activity of an academic emergency department. Intern Emerg Med. 2018;13:251-56.

14. Monitor vaccination coverage rate in the Netherlands in 2018 by Nivel [cited January 2020]. Available from: https://www.nivel.nl/nl/publicatie/vaccinatiegraad-nationaal-programma-grieppreventie-2018monitor-het-kort.

15. Monitor vaccination coverage rate in the Netherlands in 2019 by Nivel [cited May 2021]. Available from: https://www.nivel.nl/nl/publicatie/monitor-vaccinatiegraad-nationaal-programmagrieppreventie-2019.

16. Heins MJ, Hooiveld M, Korevaar JC. Healthy elderly and influenzavaccination. Hum Vaccin Immunother. 2018;14:2987-89.

17. de Bekker-Grob EW, Veldwijk J, Jonker M, et al. The impact of vaccination and patient characteristics on influenza vaccination uptake of elderly people: A discrete choice experiment. Vaccine. 2018;36:146776.

18. Evans MR, Watson PA. Why do older people not get immunised against influenza? A community survey. Vaccine. 2003;21:2421-7. 
19. Betsch C, Rossmann C, Pletz MW, et al. Increasing influenza and pneumococcal vaccine uptake in the elderly: study protocol for the multi-methods prospective intervention study Vaccination60. BMC Public Health. 2018;18:885.

20. Schmid P, Rauber D, Betsch C, Lidolt G, Denker ML. Barriers of Influenza Vaccination Intention and Behavior - A Systematic Review of Influenza Vaccine Hesitancy, 2005 - 2016. PLoS One. 2017;12:e0170550.

21. Evans MR, Prout H, Prior L, Tapper-Jones LM, Butler CC. A qualitative study of lay beliefs about influenza immunisation in older people. Br J Gen Pract. 2007;57:352-8.

22. Telford R, Rogers A. What influences elderly peoples' decisions about whether to accept the influenza vaccination? A qualitative study. Health Educ Res. 2003;18:743-53.

23. Wooten KG, Wortley PM, Singleton JA, Euler GL. Perceptions matter: beliefs about influenza vaccine and vaccination behavior among elderly white, black and Hispanic Americans. Vaccine. 2012;30:692734.

24. Rikin S, Scott V, Shea S, LaRussa P, Stockwell MS. Influenza Vaccination Beliefs and Practices in Elderly Primary Care Patients. J Community Health. 2018;43:201-06.

25. Bovier PA, Chamot E, Bouvier Gallacchi M, Loutan L. Importance of patients' perceptions and general practitioners' recommendations in understanding missed opportunities for immunisations in Swiss adults. Vaccine. 2001;19:4760-7.

26. Collange F, Verger P, Launay O, Pulcini C. Knowledge, attitudes, beliefs and behaviors of general practitioners/family physicians toward their own vaccination: A systematic review. Hum Vaccin Immunother. 2016;12:1282-92.

27. Bij D. De griepprik: wetenschappelijk falen en belangenverstrengeling. Huisarts en Wetenschap 2019;62.

28. Simonsen L, Taylor RJ, Viboud C, Miller MA, Jackson LA. Mortality benefits of influenza vaccination in elderly people: an ongoing controversy. Lancet Infect Dis. 2007;7:658-66.

29. Surveillance program of influenza (flunet) by WHO [cited May 2021]. Available from: https://www. whoint/influenza/gisrs_laboratory/flunet/en/.

30. Darvishian M, Dijkstra F, van Doorn E, et al. Influenza Vaccine Effectiveness in the Netherlands from 2003/2004 through 2013/2014: The Importance of Circulating Influenza Virus Types and Subtypes. PLoS One. 2017;12:e0169528.

31. Praditsuwan R, Assantachai P, Wasi C, Puthavatana P, Kositanont U. The efficacy and effectiveness of influenza vaccination among Thai elderly persons living in the community. J Med Assoc Thai. 2005;88:256-64.

32. Demicheli $V$, Jefferson T, Di Pietrantonj $C$, et al. Vaccines for preventing influenza in the elderly. Cochrane Database Syst Rev. 2018;2:Cd004876.

33. Coudeville L, BailleuxF, Riche B, Megas F,Andre P, Ecochard R. Relationship between haemagglutinationinhibiting antibody titres and clinical protection against influenza: development and application of a bayesian random-effects model. BMC Med Res Methodol. 2010;10:18.

34. Knottnerus JA. Influenza vaccination in the elderly: current evidence and uncertainties. J Clin Epidemiol. 2009;62:675-6.

35. Baxter R, Lee J, Fireman B. Evidence of bias in studies of influenza vaccine effectiveness in elderly patients. J Infect Dis. 2010;201:186-9.

36. Jackson LA, Jackson ML, Nelson JC, Neuzil KM, Weiss NS. Evidence of bias in estimates of influenza vaccine effectiveness in seniors. Int J Epidemiol. 2006;35:337-44.

37. Thomas RE, Lorenzetti DL. Interventions to increase influenza vaccination rates of those 60 years and older in the community. Cochrane Database Syst Rev. 2018;5:Cd005188. 
38. Martinez-Baz I, Aguilar I, Moran J, Albeniz E, Aldaz P, Castilla J. Factors associated with continued adherence to influenza vaccination in the elderly. Prev Med. 2012;55:246-50.

39. Frank E, Dresner Y, Shani M, Vinker S. The association between physicians' and patients' preventive health practices. Cmaj. 2013;185:649-53.

40. Monitor vaccination coverage rate in the Netherlands in 2017 by Nivel [cited January 2020]. Available from: https://www.nivel.nl/nl/publicatie/vaccinatiegraad-nationaal-programma-grieppreventie-2017monitor-het-kort.

41. Govaert TM, Sprenger MJ, Dinant GJ, Aretz K, Masurel N, Knottnerus JA. Immune response to influenza vaccination of elderly people. A randomized double-blind placebo-controlled trial. Vaccine. 1994;12:1185-9.

42. Thijs C, Beyer WE, Govaert PM, Sprenger MJ, Dinant GJ, Knottnerus A. Mortality benefits of influenza vaccination in elderly people. Lancet Infect Dis. 2008;8:460-1; author reply 63-5. 



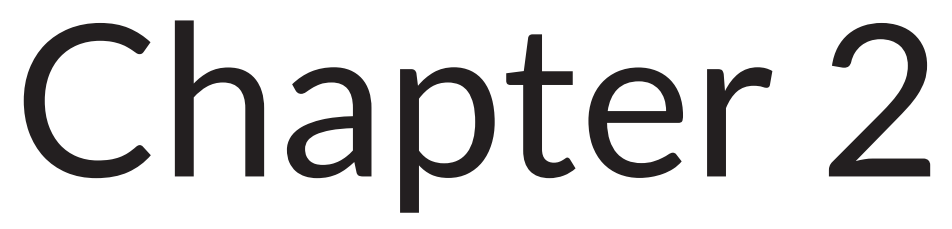

\section{Influenza vaccination in the elderly: is a trial on mortality ethically acceptable?}

Ruud A.F. Verhees

Wybo J. Dondorp

Carel Thijs

Geert-Jan Dinant

Johannes A. Knottnerus

Vaccine 2018;36:2991-7

Ned Tijdschr Geneeskd. 2018;162:D3177 


\section{ABSTRACT}

The effectiveness of influenza vaccination in the elderly has long been a topic of debate, fuelled by the absence of direct evidence from randomized trials on its effect on mortality and the methodological limitations of observational studies pointing this direction. It is argued that new placebo-controlled trials should be undertaken to resolve this uncertainty. However, such trials may be ethically questionable. To bring this discussion forward, we provide a comprehensive overview of the ethical challenges of an influenza vaccine efficacy trial designed to evaluate mortality in the elderly. An important condition in the justification of a trial is the existence of genuine uncertainty in regard to the answer to a research question. Therefore an extensive analysis of the existing levels of knowledge is needed to support the conclusion that an effect of vaccination on mortality is uncertain. Even if a so called 'clinical equipoise' status applies, denying a control group vaccination would be problematic because vaccination is considered 'competent care' and withholding vaccination could substantially increase patients' risk for influenza and its complications. Given the high burden of disease and already proven benefits of vaccination, such a trial is unlikely to meet the Declaration of Helsinki stating that the importance of a trial must outweigh the risk patients are exposed to. While a placebo-controlled trial in vaccine refusers may be considered, such a trial is unlikely to meet substantial methodological barriers regarding trial size and generalizability. We conclude that a new trial is unlikely to provide for a direct answer, let alone change current policy. At the same time, given the lack of consensus on the ethical acceptability of a placebo-controlled trial on the effect of influenza vaccination on mortality in the elderly, we invite researchers considering such trials to address the ethical challenges discussed in this manuscript. 


\section{Introduction}

The first scientific papers addressing influenza vaccination were written nearly one century ago. ${ }^{1}$ Nowadays, its relevance to the indicated risk groups and its effects on prevention of influenza and mortality is still debated..$^{2-4}$ Given the limitations of the available evidence, critics state that large placebo-controlled randomized clinical trials (RCTs) should be undertaken to resolve uncertainty regarding its effects in those aged 65 years or older. ${ }^{5,6}$ In particular, such trials could clarify the relation between vaccination and mortality in the elderly, since direct evidence from trials on this outcome is lacking.? Some consider a new placebo-controlled trial unethical. ${ }^{8-10}$ However, a comprehensive ethical analysis on the ethical acceptability of an influenza trial on mortality has never been published. Such an analysis could help to move the discussion forward and prepare for future evaluation of new influenza vaccines. In this essay, the scientific context of influenza vaccination in the elderly is discussed. We focus on the ethical and scientific challenges of a new placebo-controlled RCT designed to evaluate the efficacy of influenza vaccination in the elderly on mortality, and explore whether or not these challenges can be overcome.

\section{Scientific context of influenza vaccination}

Observational studies

Studies evaluating the effectiveness of influenza vaccination in the elderly are mainly observational, regardless the endpoints used. In observational studies, the risk of bias and confounding is increased because of differences in characteristics between vaccinated and unvaccinated groups. 2,9,11 Moreover, indirect, subjective and non-specific outcome measures are often used to determine the effect on prevention of influenza and the complications attributed to influenza. This hinders the interpretation of studies and leaves researchers to draw different conclusions for various endpoints. Epidemiologists attempt to minimize bias in observational studies by various means. For instance, using a test-negative design, researchers reported a $44 \%$ reduction of influenza in vaccinated elderly. ${ }^{12}$ Although vaccination is likely to prevent morbidity to this extent, cohort studies on mortality often report similar reductions in the total risk of death in winter, whereas in fact (only) $5 \%$ of all winter deaths is attributed to influenza. ${ }^{2}$ Since influenza vaccination cannot reasonably be expected to do any more than eliminate this excess $5 \%$ mortality, this questions whether observational studies can adequately assess the impact of vaccination on mortality.

\section{Placebo-controlled trials}

Prevention of influenza is an intermediate outcome in preventing its' complications and mortality. Available evidence on the efficacy of influenza vaccination on prevention of influenza shows that only three RCTs have been conducted among community-dwelling elderly. ${ }^{13-15}$ Two trials showed a reduction of about $50 \%$ on clinical influenza. ${ }^{13,15}$ The third reported a decrease in self-reported influenza-like illness (ILI), but not on doctor's diagnosis of ILI or pneumonia. ${ }^{14}$ Trials conducted in elderly with chronic diseases usually do not report on prevention of influenza but focus on 
averting complications by vaccination. A placebo-controlled trial that included Thai elderly with chronic obstructive pulmonary disease before free provision of influenza vaccination was introduced, demonstrated a reduction in ILI of 66\%. ${ }^{16}$ Up to our best knowledge, only two placebo-controlled trials reporting on the preventive effect of vaccination on influenza have been conducted in older adults (the majority $\leq 60$ years old) with known cardiovascular disease, both demonstrating significant preventive effects. ${ }^{17,18}$ No RCTs on the prevention of influenza among elderly diabetics or institutionalized elderly have been published. Trials large enough to draw conclusions on the efficacy of influenza vaccination in the elderly on mortality attributed to influenza are non-existent.

\section{Meta-analyses and systematic reviews}

Meta-analyses and systematic reviews suggest effects of vaccination in elderly with or without comorbidity in preventing influenza 8, 12,19, 20 and mortality. ${ }^{19-21}$ However, many studies used in these analyses are biased and of poor quality regarding endpoints such as pneumonia, hospitalization and mortality. ${ }^{7}$ Use of innovative methods to minimize bias yields an unrealistic $36 \%$ reduction of total mortality by vaccination. ${ }^{22}$ These methodologic limitations have held back some authors from drawing unambiguous conclusions. ${ }^{5,23}$ Meta-analyses solely based on trials are sparse and mainly include trials conducted in elderly with chronic diseases. ${ }^{24-26}$ For instance, Clar et al. concluded that vaccination might reduce cardiovascular mortality in (largely) elderly patients with coronary heart disease. ${ }^{26}$ For a complete overview of the available literature on this topic, we refer to the recently updated meta-analysis by Demichelli et al. ${ }^{6}$

International and individual viewpoints

The scientific literature guides expert decision and policy making. The World Health Organization (WHO), Advisory Committee on Immunization Practices (ACIP) of the Centers for Disease Control and Prevention (CDC), and the European Centre for Disease Prevention and Control (ECDC) unanimously recommend influenza vaccination, especially in high-risk groups such as elderly and patients with a known medical indication. ${ }^{10,27-29}$ Worldwide, medical associations support these recommendations or have included them in their clinical guidelines.

Approximately $90 \%$ of the physicians think influenza vaccination in the elderly is effective ${ }^{30,31}$ and $85 \%$ of the general practitioners (GPs) recommend influenza vaccination to their elderly patients. ${ }^{32,33}$ Of those GPs that do not advise vaccination, 17\% question the effectiveness of vaccination. ${ }^{32}$ About $70 \%$ of the community-dwelling elderly with a medical indication believe vaccination is effective. ${ }^{34-36}$ When patients refuse vaccination, this is mainly because they consider themselves healthy, ${ }^{35-37}$ or fear side effects. ${ }^{38}$ Vaccination is refused due to uncertainty regarding its effectiveness in only $2 \%$ of the high-risk patients ${ }^{36}$ and approximately $5 \%$ of the community-dwelling elderly aged $\geq 65 .^{39,40}$ It should be noted that none of the cited studies mention to which endpoint the belief in effectiveness applies. 
Apart from personal belief, also differences in costs and benefits estimations, demography, age criteria for vaccination and policies affect the actual coverage rates for influenza vaccination in the elderly. In the European countries ${ }^{41}$ as well as the United States (coverage rate 57\%) ${ }^{42}$ coverage rates are well below the $75 \%$ target set as minimum by the $\mathrm{WHO}$.

\section{A placebo-controlled trial on mortality: can the case be made?}

Limitations in the available evidence as discussed leave a number of authors to conclude that a new placebo-controlled trial could reduce uncertainty regarding the effect of influenza vaccination in the elderly on mortality. ${ }^{5,43,44}$ In the following sections we provide a comprehensive overview of the challenges that determine the ethical acceptability of a trial designed to evaluate the effect of influenza vaccination in the elderly on mortality as an endpoint. These challenges are summarized in Fig.1. We note that a clear distinction between elderly with and without comorbidity cannot always be made since chronic diseases are not always diagnosed. ${ }^{45-47}$

\section{Existing knowledge and the relevance of uncertainty}

Informed and voluntary participation are often considered the central tenet of clinical research. ${ }^{48}$ However, in terms of ethical acceptability, these are necessary but not sufficient conditions. An essential but often overlooked condition is the scientific justification for conducting the intended research. ${ }^{49}$ Evaluation of the scientific justification of a trial requires extensive understanding of the existing knowledge on three different levels: the scientific literature, the prevailing expert opinion and insights of individual researchers. ${ }^{50}$ Based on our review of literature, the existing knowledge concerning the effect of vaccination on mortality can be summarized as follows. There are multiple observational studies, systematic reviews and meta-analyses that support a beneficial effect of vaccination on mortality, but the evidence is predominantly of poor quality. ${ }^{7}$ Since vaccination prevents influenza, it is plausible that vaccination also prevents severe complications related to influenza (e.g. pneumonia, heart failure and myocardial infarction) ${ }^{26}$, 51,52 and mortality. ${ }^{53}$ Expert opinion, as reflected by leading health organisations and medical associations, states that influenza vaccination as such should be recommended. However, some health organisations are cautious in ascribing effects of vaccination to mortality reduction. ${ }^{54}$ Finally, the lack of direct evidence on an effect on mortality as well as the methodological limitations of observational studies, have yielded different insights among researchers. 3, 5, 55 Points addressed in this summary correspond with the top four diamonds in Fig.1. 


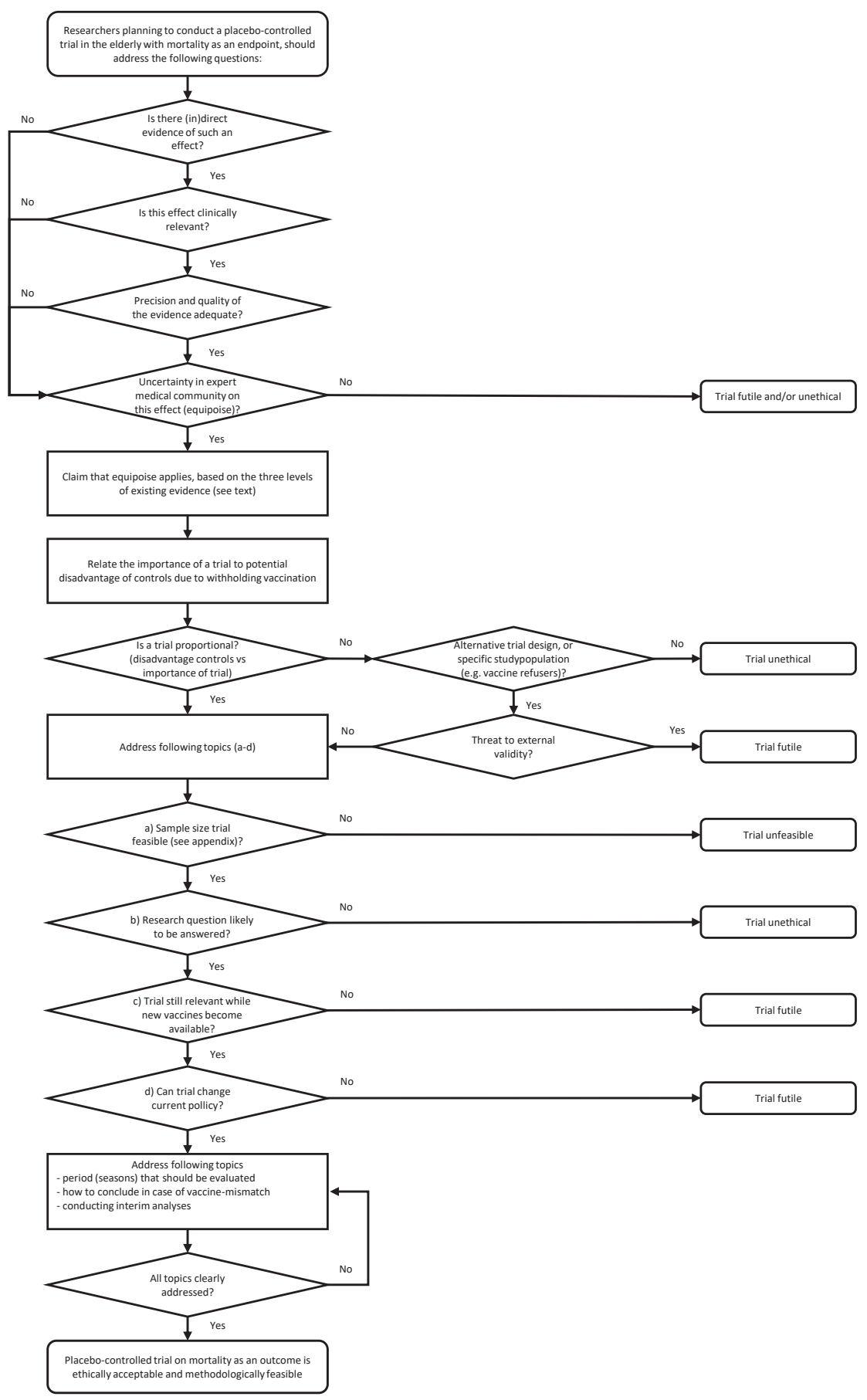

Fig.1. Flow chart indicating the ethical and methodological challenges of a hypothetical placebocontrolled influenza vaccination trial in the elderly with mortality as an endpoint. 
Proponents of a trial should take all levels of knowledge into account, when concluding on the level of existing uncertainty regarding the effect on mortality. Existence of genuine uncertainty in the expert medical community about a preferred treatment (or prevention option) is defined by Freedman as 'clinical equipoise.' ${ }^{56}$ Equipoise is still considered a vital condition in the ethical approval of trials since it guarantees that no participant receives care known to be inferior to any available alternative. ${ }^{57}$ In practice, the level of equipoise (reflected by the proportion of experts favoring either one of the intervention options) determines eventual ethical approval. ${ }^{58}$ Therefore, in order to conduct a trial on mortality, it is essential that trial proponents can demonstrate equipoise exists on this endpoint.

In contrast to the effect of influenza vaccination in the elderly on mortality, the effect on prevention of influenza was proven by the highest degree of evidence. ${ }^{7}$ Health institutes, professional medical associations, practicing physicians and elderly patients seem to support influenza vaccination to a high extent. Thus, it seems difficult to maintain that there is equipoise regarding the effect of vaccination on preventing influenza. Therefore, influenza vaccination is considered 'competent care'; it encompasses the norms for practice that derive from practices accepted by the community of expert practitioners. ${ }^{59}$ Since physicians are obliged to provide their patients with competent care, influenza vaccination cannot be denied to the elderly in a research setting. Denying vaccination fails to meet the principle of beneficence since this would substantially increase their risk for influenza and its possible complications. It is also unjust, as it denies them what they are entitled to.

We conclude that if trial proponents make the case that an effect of vaccination on mortality is uncertain, a trial aimed at filling this gap might gain scientific justification. As it seems difficult to maintain that a similar case can be made regarding an effect of vaccination on morbidity, this raises a significant barrier for the acceptability of such a trial. Inevitably, a placebo-controlled trial would withhold competent care from the control group, regardless of the endpoint being assessed. Since scientific justification might be present for a trial with mortality as an endpoint, trial proponents might not yet acquiesce to the conclusion that such a trial is ethically unacceptable. Their case might be made by arguing that any concerns related to withholding vaccination are not weighty enough to stand in the way of a trial that will yield important knowledge.

\section{Balance between risks and benefits}

According to the Declaration of Helsinki, medical research may only be conducted if the objective's importance outweighs the risks and burdens to the research subjects. ${ }^{60}$ Since a placebo-controlled trial increases the risk of influenza in controls, a trial will therefore only meet this condition if trial proponents can relativize this risk while emphasizing the importance of a trial. 
The risk for the placebo-group can be relativized in two ways: (1) by denying that influenza vaccination should be considered as competent care, or (2) by relativizing the medical importance of preventing influenza. Following the first line of argument, trial proponents could state that observational research shows that mismatches between vaccines and actual circulating viruses may decrease vaccine effectiveness. ${ }^{61}$ However, the previously mentioned randomized trials, ${ }^{13}$, ${ }^{15}$ point estimates of smaller trials ${ }^{5}$ and current viewpoints of medical associations, place the burden of proof at the plate of trial proponents. In order to make a successful claim to equipoise, the existing evidence should be refuted in such a way that medical associations (representing the 'expert community') will have to reconsider their viewpoints. In order to relativize the medical importance of vaccination (the second possible line of argument), trial proponents should set out why being deprived of a potential 50\% reduction in the risk of getting influenza is not a serious drawback that would hinder the ethical acceptability of a placebo-controlled trial. We do think that this would be difficult to maintain given the high burden of disease caused by influenza. ${ }^{62,63}$

In addition to downplaying the consequences for controls, proponents would have to present the importance of a new trial as unescapable, in order to make the case that any remaining adverse effects for controls can be considered proportional. Here, the argument might be that a negative trial (showing no effect on mortality) would provide an argument for reconsidering the time and resources spent on vaccination programs. However, if no effect on mortality is found in a sufficiently powered trial, the clinical implication would still be questionable given the effect of vaccination on influenza. Before a template to guide vaccine policy making was suggested, ${ }^{64}$ the Health Council of the Netherlands set up a framework to assess whether specific vaccination of specific target groups would be useful, in order to support policy making. ${ }^{65}$ Influenza vaccination of the elderly met these criteria. For example, vaccination can reduce the significant loss of productivity imposed by influenza. ${ }^{66,67}$ Also, taking in account the high economic burden of an influenza epidemic (up to $\$ 87$ billion in the US) ${ }^{68}$ and the annual vaccination program costs ( $\$ 1.7$ billion in the US), ${ }^{69}$ vaccination is considered cost effective in patients with a medical indication, ${ }^{70}$ elderly aged 65 or older ${ }^{69}$ and those aged $50-64 .{ }^{71}$ All in all, previously mentioned benefits of influenza vaccination remain relevant arguments in the justification of current policies, also when a new trial would not demonstrate an effect on mortality.

Trial proponents might also state that a new trial is needed because all medical practice and prevention must be based on direct RCT evidence. This might account for vaccines especially since these are offered to 'healthy' individuals. ${ }^{72}$ However, many accepted recommendations for medical care and prevention are based on intermediate outcomes. Medical and preventive practice would be severely restricted if not become impossible when direct RCT-based knowledge would be required for all final outcomes. In many cases it is clear beforehand that RCT evidence in direct relation to endpoints such as mortality is hard or even impossible to gain, thus practitioners and policy makers have to act in a context of sparse or absent RCT evidence. Moreover, many years of influenza vaccination in several million people have yielded valuable observational data on for instance vaccination safety that could impossibly be provided by trials. 
Taking everything into account, we do not think that an argumentative strategy of downplaying the benefits of vaccination as disease prevention while emphasizing the importance of a future placebo-controlled trial aimed at determining its effect on mortality can be successful. Although one can argue on the equipoise status of the effect of vaccination on mortality, the competent care dilemma is unlikely to be overcome, which means that the risks will outweigh the benefits of a trial. As a new placebo-controlled trial on mortality is unlikely to meet the Declaration of Helsinki, the conclusions must be that such a trial is ethically problematic.

\section{Methodological constraints of a new RCT}

Ethical constraints that arise from denying patients competent care can be avoided if only vaccine refusers would be asked to participate in a trial. For instance, currently a placebocontrolled influenza vaccination trial is being conducted among patients admitted with myocardial infarction who do not plan to receive influenza vaccination during admission. ${ }^{73}$ Although inclusion of vaccine refusers seems permissible, this might add up to some of the already existing threats to the methodological rigor of a trial, as will become clear from the following paragraph.

A trial that evaluates an infrequent endpoint requires many participants to be included. Sample sizes for an influenza trial on mortality can range widely, depending on the preferred statistical power and level of significance, and the assumed mortality attributed to influenza. Under reasonable trial conditions and assumptions, at least half a million elderly must be included in a trial, in order to prove an effect of influenza vaccination on mortality related to influenza (sample size calculations are available as Supplement). Another methodological challenge lies in the study period during which the efficacy of the vaccine is to be assessed. Since the incidence of influenza varies annually and vaccine mismatches occur regularly, ${ }^{61}$ results of a trial covering more than one season will be more externally valid. ${ }^{74}$ Now, a new ethical question arises: should the equipoise status be adjusted based on knowledge derived after each separate season (as in interim analyses) or should mortality data be only analyzed when all preplanned seasons are over? A practical problem also concerns contamination which can easily occur in participants favoring influenza vaccination, since vaccination is still (freely) accessible. Practical issues may even become larger when solely vaccine refusers are selected for inclusion since fewer people will now fit the inclusion criteria and refusers might be less willing to participate considering their preference not to be vaccinated. Also, the generalizability of such a trial might be limited since characteristics of vaccine refusers may differ from those getting their vaccination. ${ }^{75}$ It should be clear that if research on forehand is unlikely to answer the research question due to related methodological constraints, such research is not ethically permissible. 


\section{Future influenza related research}

Progress is being made in development of new influenza vaccines such as recombinant vaccines

${ }^{76}$ or a universal vaccine. ${ }^{77}$ Besides the regular (standard-dose) vaccine, now the adjuvanted and the high-dose vaccine have been registered for the elderly. However, claims on the immunogenicity (the ability to induce an immune response) and effectiveness of new and more complex vaccines can increase constraints for performing a new placebo-controlled trial.

More participants will be needed as multiple study arms will have to be considered (registered vaccine(s), new vaccine, placebo), and also because differences on endpoints between the active intervention groups could be relatively small. ${ }^{53}$ For this latter reason, also head-to-head trials may have to include unrealistically high numbers of participants to meet the sample size required to prove superiority of a new vaccine over the regular vaccine on mortality as an endpoint. Although the CDC has not yet expressed a preference for any influenza vaccine, even a trial that compares a new vaccine to the standard-dose vaccine could soon become troublesome from an ethical perspective, since multiple vaccine types have already been proven superior to standard dose vaccination in the past few years..$^{76,78}$

\section{Conclusion}

The scientific urge to increase the existing knowledge by conducting a placebo-controlled trial on the effects of influenza vaccination in the elderly on mortality is understandable. However, we cannot escape from critically reviewing the ethical and scientific considerations that pose a threat to the acceptability of such a trial (summarized in Fig.1). Based on our analysis we conclude that a new placebo-controlled trial evaluating the effects of mortality in the elderly is ethically problematic. At the same time, given the lack of consensus on the ethical acceptability of conducting such a trial, we invite researchers who support or consider such trials, to challenge the ethical analysis provided in this essay. 


\section{REFERENCES}

1. Heagerty JJ. Influenza and vaccination. Can Med Assoc J 1919;9:226-8.

2. Simonsen L, Taylor RJ, Viboud C, Miller MA, Jackson LA. Mortality benefits of influenza vaccination in elderly people: an ongoing controversy. Lancet Infect Dis 2007;7:658-66.

3. McCartney M. Margaret McCartney: what use is mass flu vaccination? BMJ 2014;349:g6182.

4. van der Wouden JC, Bueving HJ, Poole P. Preventing influenza: an overview of systematic reviews. Respir Med 2005;99:1341-9.

5. Jefferson T, Di Pietrantonj C, Al-Ansary LA, Ferroni E, Thorning S, Thomas RE. Vaccines for preventing influenza in the elderly. Cochrane Database Syst Rev 2010:CD004876.

6. Demicheli V, Jefferson T, Di Pietrantonj C, et al. Vaccines for preventing influenza in the elderly. Cochrane Database Syst Rev 2018;2:Cd004876.

7. Michiels B, Govaerts F, Remmen R, Vermeire E, Coenen S. A systematic review of the evidence on the effectiveness and risks of inactivated influenza vaccines in different target groups. Vaccine 2011;29:9159-70.

8. Darvishian M, Bijlsma MJ, Hak E, van den Heuvel ER. Effectiveness of seasonal influenza vaccine in community-dwelling elderly people: a meta-analysis of test-negative design case-control studies. Lancet Infect Dis 2014;14:1228-39.

9. Baxter R, Lee J, Fireman B. Evidence of bias in studies of influenza vaccine effectiveness in elderly patients. J Infect Dis 2010;201:186-9.

10. Information for Professionals on vaccination effectiveness by CDC [cited 2018 January]. Available from: https://www.cdcgov/flu/professionals/vaccination/effectiveness.

11. Jackson LA, Jackson ML, Nelson JC, Neuzil KM, Weiss NS. Evidence of bias in estimates of influenza vaccine effectiveness in seniors. Int J Epidemiol 2006;35:337-44.

12. Darvishian $M$, van den Heuvel ER, Bissielo $A$, et al. Effectiveness of seasonal influenza vaccination in community-dwelling elderly people: an individual participant data meta-analysis of test-negative design case-control studies. Lancet Respir Med 2017;5:200-11.

13. Govaert TM, Thijs CT, Masurel N, Sprenger MJ, Dinant GJ, Knottnerus JA. The efficacy of influenza vaccination in elderly individuals A randomized doubleblind placebo-controlled trial. JAMA 1994;272:1661-5.

14. Allsup S, Haycox A, Regan M, Gosney M. Is influenza vaccination cost effective for healthy people between ages 65 and 74 years? A randomised controlled trial. Vaccine 2004;23:639-45.

15. Praditsuwan R, Assantachai P, Wasi C, Puthavatana P, Kositanont U. The efficacy and effectiveness of influenza vaccination among Thai elderly persons living in the community. J Med Assoc Thai 2005;88:256-64.

16. Wongsurakiat P, Maranetra KN, Wasi C, Kositanont U, Dejsomritrutai W, Charoenratanakul S. Acute respiratory illness in patients with COPD and the effectiveness of influenza vaccination: a randomized controlled study. Chest 2004;125:2011-20.

17. Ciszewski A, Bilinska ZT, Brydak LB, et al. Influenza vaccination in secondary prevention from coronary ischaemic events in coronary artery disease: FLUCAD study. Eur Heart J 2008;29:1350-8.

18. Keshtkar-Jahromi M. The efficacy of influenza vaccination in reducing cardiovascular events in patients with coronary artery diseases: IVCAD study. Clin Microbiol Infect 2009;15:395 [unpublished].

19. Beyer WE, McElhaney J, Smith DJ, Monto AS, Nguyen-Van-Tam JS, Osterhaus AD. Cochrane rearranged: support for policies to vaccinate elderly people against influenza. Vaccine 2013;31:6030-3. 
20. Nichol KL. Influenza vaccination in the elderly: impact on hospitalisation and mortality. Drugs Aging 2005;22:495-515.

21. Chan TC, Fan-Ngai Hung I, Ka-Hay Luk J, Chu LW, Hon-Wai Chan F. Effectiveness of influenza vaccination in institutionalized older adults: a systematic review. J Am Med Dir Assoc. 2014;15:226. e1-6.

22. Darvishian M, Gefenaite G, Turner RM, et al. After adjusting for bias in meta-analysis seasonal influenza vaccine remains effective in community-dwelling elderly. J Clin Epidemiol 2014;67:734-44.

23. Remschmidt C, Wichmann $O$, Harder T. Vaccines for the prevention of seasonal influenza in patients with diabetes: systematic review and meta-analysis. BMC Med 2015;13:53.

24. Poole PJ, Chacko E, Wood-Baker RW, Cates CJ. Influenza vaccine for patients with chronic obstructive pulmonary disease. Cochrane Database Syst Rev. 2006:Cd002733.

25. Udell JA, Zawi R, Bhatt DL, Keshtkar-Jahromi M, Gaughran F, Phrommintikul A, et al. Association between influenza vaccination and cardiovascular outcomes in high-risk patients: a meta-analysis. JAMA 2013;310:1711-20.

26. Clar C, Oseni Z, Flowers N, Keshtkar-Jahromi M, Rees K. Influenza vaccines for preventing cardiovascular disease. Cochrane Database Syst Rev 2015;5: CD005050.

27. Fiore AE, Uyeki TM, Broder K, et al. Prevention and control of influenza with vaccines: recommendations of the Advisory Committee on Immunization Practices (ACIP), 2010. MMWR Recomm Rep 2010;59:162.

28. Comment ECDC on the effects of vaccines against influenza in healthy adults [cited January 2018]. Available from: http://ecdceuropaeu/en/activities/sciadvice/_layouts/ forms/Review_ DispFormaspx?List=a3216f4cf040-4f51-9f77-a96046dbfd72\&ID=433.

29. Influenza vaccine use by WHO [cited February 2018]. Available from: http://www.whoint/influenza/ vaccines/use/en.

30. Ridda I, Lindley IR, Gao Z, McIntyre P, Macintyre CR. Differences in attitudes, beliefs and knowledge of hospital health care workers and community doctors to vaccination of older people. Vaccine 2008;26:5633-40.

31. Pavia M, Foresta MR, Carbone V, Angelillo IF. Influenza and pneumococcal immunization in the elderly: knowledge, attitudes, and practices among general practitioners in Italy. Public Health 2003;117:2027.

32. Klett-Tammen CJ, Krause G, von Lengerke T, Castell S. Advising vaccinations for the elderly: a crosssectional survey on differences between general practitioners and physician assistants in Germany. BMC Fam Pract 2016;17:98.

33. Nichol KL, Zimmerman R. Generalist and subspecialist physicians' knowledge, attitudes, and practices regarding influenza and pneumococcal vaccinations for elderly and other high-risk patients: a nationwide survey. Arch Intern Med 2001;161:2702-8.

34. Findlay PF, Gibbons YM, Primrose WR, Ellis G, Downie G. Influenza and pneumococcal vaccination: patient perceptions. Postgrad Med J 2000;76:215-7.

35. Evans MR, Watson PA. Why do older people not get immunised against influenza? A community survey. Vaccine 2003;21:2421-7.

36. Santos AJ, Kislaya I, Machado A, Nunes B. Beliefs and attitudes towards the influenza vaccine in highrisk individuals. Epidemiol Infect 2017;145:1786-96.

37. Canova L, Birchmeier M, D'Acremont V, et al. Prevalence rate and reasons for refusals of influenza vaccine in elderly. Swiss Med Wkly 2003;133:598-602. 
38. Influenza vaccination and self-reported reasons for not receiving influenza vaccination among Medicare beneficiaries aged > or $=65$ years-United States, 1991-2002. MMWR Morb Mortal Wkly Rep. 2004;53:1012-5.

39. Dip RM, Cabrera MA. Influenza vaccination in non-institutionalized elderly: a population-based study in a medium-sized city in Southern Brazil. Cad Saude Publica 2010;26:1035-44.

40. Burns VE, Ring C, Carroll D. Factors influencing influenza vaccination uptake in an elderly, communitybased sample. Vaccine 2005;23:3604-8.

41. Influenza vaccination rates Europe by Eurostat [cited January 2018]. Available from: http:// appssoeurostateceuropaeu/nui/showdo?dataset=hlth_ps_immu\&lang=en.

42. Influenza vaccination rate US by CDC [cited January 2018]. Available from: https://www.cdcgov/flu/ fluvaxview/nifs-estimates-nov2016htm.

43. Trucchi C, Paganino C, Orsi A, De Florentiis D, Ansaldi F. Influenza vaccination in the elderly: why are the overall benefits still hotly debated? J Prev Med Hyg 2015;56:E37-43.

44. Thomas RE. Are influenza-associated morbidity and mortality estimates for those $\geq 65$ in statistical databases accurate, and an appropriate test of influenza vaccine effectiveness? Vaccine 2014;32:6884901.

45. Izaks GJ, Westendorp RG. III or just old? Towards a conceptual framework of the relation between ageing and disease. BMC Geriatr 2003;3:7.

46. Hancock HC, Close H, Mason JM, et al. High prevalence of undetected heart failure in long-term care residents: findings from the Heart Failure in Care Homes ( $\mathrm{HFinCH}$ ) study. Eur J Heart Fail 2013;15:158-65.

47. Gibson PG, McDonald VM, Marks GB. Asthma in older adults. Lancet 2010;376:803-13.

48. Shamy M, Fedyk M. Clinical Trials Involving Hypertension. N Engl J Med 2017;376:289-90.

49. Shamy M, Fedyk M. Why the ethical justification of randomized clinical trials is a scientific question. J Clin Epidemiol 2018.

50. Knottnerus JA. Gezondheidszorg in extramurale settings. Ethiek en Recht in de Gezondheidszorg 1997;4:151-94.

51. Nichol KL, Wuorenma J, von Sternberg T. Benefits of influenza vaccination for low-, intermediate-, and high-risk senior citizens. Arch Intern Med 1998;158:1769-76.

52. Kwong JC, Schwartz KL, Campitelli MA, et al. Acute Myocardial Infarction after Laboratory-Confirmed Influenza Infection. N Engl J Med 2018;378:345-53.

53. Knottnerus JA. Influenza vaccination in the elderly: current evidence and uncertainties. J Clin Epidemiol 2009;62:675-6.

54. Patient information on influenza vaccines by CDC [cited January 2018]. Available from: https:// www. cdcgov/flu/about/qa/vaccineeffecthtm.

55. Eurich DT, Marrie TJ, Johnstone J, Majumdar SR. Mortality reduction with influenza vaccine in patients with pneumonia outside "flu" season: pleiotropic benefits or residual confounding? Am J Respir Crit Care Med 2008;178:527-33.

56. Freedman B. Equipoise and the ethics of clinical research. N Engl J Med 1987;317:141-5.

57. London AJ. Equipoise in research: integrating ethics and science in human research. JAMA 2017;317:525-6.

58. Mhaskar R, BB B, Djulbegovic B. At what level of collective equipoise does a randomized clinical trial become ethical for the members of institutional review board/ethical committees? Acta Inform Med 2013;21:156-9.

59. Miller PB, Weijer C. Rehabilitating equipoise. Kennedy Inst Ethics J 2003;13:93-118. 
60. World Medical Association Declaration of Helsinki. ethical principles for medical research involving human subjects. JAMA 2013;310:2191-4.

61. Darvishian M, Dijkstra F, van Doorn E, et al. Influenza vaccine effectiveness in the Netherlands from 2003/2004 through 2013/2014: the importance of circulating influenza virus types and subtypes. PLoS One 2017;12:e0169528.

62. van Lier A, McDonald SA, Bouwknegt M, et al. Disease Burden of 32 infectious diseases in the Netherlands, 2007-2011. PLoS One 2016;11:e0153106.

63. van Asten L, van den Wijngaard C, van Pelt W, et al. Mortality attributable to 9 common infections: significant effect of influenza A, respiratory syncytial virus, influenza B, norovirus, and parainfluenza in elderly persons. J Infect Dis 2012;206:628-39.

64. Poland GA, Marcuse EK. Developing vaccine policy: attributes of "just policy" and a proposed template to guide decision and policy making. Vaccine 2011;29:7577-8.

65. Health Council of the Netherlands. Influenza vaccination: revision of the indication. The Hague: Health Council of the Netherlands. 2007; publication no. 2007/09.

66. Keech M, Beardsworth P. The impact of influenza on working days lost: a review of the literature. Pharmacoeconomics 2008;26:911-24.

67. Nichol KL, D'Heilly SJ, Greenberg ME, Ehlinger E. Burden of influenza-like illness and effectiveness of influenza vaccination among working adults aged 50-64 years. Clin Infect Dis 2009;48:292-8.

68. Molinari NA, Ortega-Sanchez IR, Messonnier ML, et al. The annual impact of seasonal influenza in the US: measuring disease burden and costs. Vaccine 2007;25:5086-96.

69. Carias C, Reed C, Kim IK, et al. Net costs due to seasonal influenza vaccination-United States, 20052009. PLoS One 2015;10:e0132922.

70. Ting EE, Sander B, Ungar WJ. Systematic review of the cost-effectiveness of influenza immunization programs. Vaccine 2017;35:1828-43.

71. Turner DA, Wailoo AJ, Cooper NJ, Sutton AJ, Abrams KR, Nicholson KG. The cost-effectiveness of influenza vaccination of healthy adults 50-64 years of age. Vaccine 2006;24:1035-43.

72. Verweij M, Dawson A. Ethical principles for collective immunisation programmes. Vaccine 2004;22:3122-6.

73. Frobert $O$, Gotberg M, Angeras $O$, et al. Design and rationale for the Influenza vaccination After Myocardial Infarction (IAMI) trial. A registry-based randomized clinical trial. Am Heart J 2017;189:94102.

74. Jefferson T. Influenza vaccination: policy versus evidence. BMJ 2006;333:912-5.

75. Ward L, Draper J. A review of the factors involved in older people's decision making with regard to influenza vaccination: a literature review. J Clin Nurs 2008;17:5-16.

76. Dunkle LM, Izikson R, Patriarca P, et al. Efficacy of Recombinant Influenza Vaccine in Adults 50 Years of Age or Older. N Engl J Med 2017;376:2427-36.

77. de Vries RD, Nieuwkoop NJ, van der Klis FRM, Koopmans MPG, Krammer F, Rimmelzwaan GF. Primary human influenza B virus infection induces crosslineage hemagglutinin stalk-specific antibodies mediating antibodydependent cellular cytoxicity. J Infect Dis 2017;217:3-11.

78. DiazGranados CA, Dunning AJ, Kimmel M, et al. Efficacy of high-dose versus standard-dose influenza vaccine in older adults. N Engl J Med 2014;371:635-45. 


\section{SUPPLEMENTARY INFORMATION}

Sample size calculation for a placebo-controlled trial evaluating the effect of influenza vaccination in the elderly on mortality related to influenza (by different approaches).

Approach 1: Assuming the incidence of influenza-related mortality during winter ranges from 1/500 to $1 / 2,000$ elderly.

\begin{tabular}{llrr}
\hline Incidence & Vaccine Efficacy (VE) & $\beta=0.2$ & $\beta=0.1$ \\
\hline $1 / 500$ & 0.5 & 47,321 & 62,895 \\
& 0.25 & 220,805 & 293,475 \\
& 0.1 & $1,498,140$ & $1,991,199$ \\
& 0.05 & $6,149,976$ & $8,174,019$ \\
\hline $1 / 1,000$ & 0.5 & 94,721 & 125,895 \\
& 0.25 & 442,005 & 587,475 \\
& 0.1 & $2,999,140$ & $3,986,199$ \\
& 0.05 & $12,311,976$ & $16,364,019$ \\
\hline $1 / 2,000$ & 0.5 & 189,521 & 251,895 \\
& 0.25 & 884,405 & $1,175,475$ \\
& 0.1 & $6,001,140$ & $7,976,199$ \\
& 0.05 & $24,635,976$ & $32,744,019$ \\
\hline
\end{tabular}

Approach 2.1: Assuming mortality attributed to influenza $=5 \%$ (of the total mortality rate)

\begin{tabular}{lll}
\hline & $\beta=0.2$ & $\beta=0.1$ \\
\hline$V E=0.5$ & $3,449,925$ & $4,585,343$ \\
$V E=0.25$ & $13,885,806$ & $18,455,818$ \\
$V E=0.1$ & $87,109,095$ & $115,777,912$ \\
$V E=0.05$ & $348,866,730$ & $463,683,629$ \\
\hline
\end{tabular}

Approach 2.2: Assuming mortality attributed to influenza $=10 \%$ (of the total mortality rate)

\begin{tabular}{lll}
\hline & $\beta=0.2$ & $\beta=0.1$ \\
\hline$V E=0.5$ & 851,712 & $1,132,022$ \\
$V E=0.25$ & $3,449,925$ & $4,585,343$ \\
$V E=0.1$ & $21,723,474$ & $28,872,972$ \\
$V E=0.05$ & $87,109,095$ & $115,777,912$ \\
\hline
\end{tabular}




\section{Calculation method and assumptions}

Power calculation formula, as by Pocock. $2008^{1}$

$n=\frac{\mathrm{p} 1 *(100-\mathrm{p} 1)+\mathrm{p} 2 *(100-\mathrm{p} 2)}{(\mathrm{p} 2-\mathrm{p} 1)^{2}} * \mathrm{f}(\alpha, \beta)$

$\mathrm{n}=$ number of patients required in each group

p1 = percentage of successes expected on placebo

p2 = percentage of successes expected on intervention (influenza vaccine)

$a=$ level of the $x^{2}$ significance test used for detecting a treatment difference

$\beta=$ probability of not detecting a significant difference when there really is a difference of magnitude

$f(a, \beta)=$ function of $\alpha$ and $\beta$, the values of which can be obtained from statistical tables ${ }^{1}$

sample size $=\mathrm{n}^{*} 2$

\section{Approach 1}

Definitions:

p1: chance for controls not to die from influenza-related illness during the winter

p2: chance for vaccinated elderly not to die from influenza-related illness during the winter

- $\quad \mathrm{p} 1=999 / 1,000=99.9 \%$ (given incidence influenza-related deaths in winter $=1 / 1,000$ elderly) ${ }^{2}$

- $\quad$ p2 $=p 1+\left((100-p 1)^{*} V E\right)=99.9+(0.1 * V E)$

- $\quad V E=$ Vaccine Efficacy on endpoint mortality (VE range: $50 \%$ to $5 \%$ )

- $\quad V E$ (endpoint influenza) $\approx 50 \%,{ }^{2}$ thus VE (endpoint mortality) $\leq 50 \%$

- Note that also a VE(endpoint mortality) $<50 \%$ can still be considered a relevant effect given the high incidence of influenza in the general population

- $\quad a=$ level of the $x^{2}$ significance test for detecting a treatment difference (set $a=0.05$ )

- $\beta=$ probability of not detecting a significant difference when there really is a difference of magnitude ( $\beta$ range: 0.2 to 0.1 ) 


\section{Approach 2.1}

Definitions:

p1: chance for controls to survive (thus not to die from any cause) potential influenza epidemic months s.\#

p2: chance for vaccinated elderly to survive (thus not to die from any cause) potential influenza epidemic months $\$$

- Mean mortality rate in the Dutch elderly population ( $\geq 60$ years) from December through April $=14.3$ deaths 1,000 elderly $\#=1.43 \%$. ${ }^{3,4}$ (Monthly range 0.25 to $0.32 \%$ )

- $\quad$ Thus $1=100 \%-1.43 \%=98.57 \%$

- Mortality related to influenza during influenza period $=5 \%{ }^{5}$ (of the $14.3 / 1,000$ )

- $\quad$ p2 $=\mathrm{p} 1+(0.05 * 1.43 \% * V E)$

- $\quad \mathrm{VE}, \alpha, \beta$ as in approach 1

§ Potential influenza epidemic months can range widely.

\# Calculated by: Sum of all elderly (aged 60 and above) deaths during the months December through April in the years 2011 through 2016. For each year the number of deaths during these months was divided by the total elderly population as known at the first of January including the total numbers of deaths during December through April. The mean mortality rate was calculated for these five consecutive years. The mortality rate in a strictly unvaccinated population is expected to be slightly higher than 14.3/1,000. Mean mortality rate in 2004/05 and 2011/12 (in which vaccine efficacy was close to zero due to vaccine-mismatch) 6 was 15.8 / 1,000 elderly 3,4 , yielding up to $10 \%$ lower sample sizes.

\section{Approach 2.2}

Definitions: as in approach 2.1

- $\quad$ p1, VE, a, $\beta$ as in approach 2.1

- Mortality related to influenza during influenza period = 10\% (of the 14.3/1,000)

- $\quad$ p2 $=$ p1 $+(0.10 * 1.43 \% * V E)$ 


\section{REFERENCES}

1. Pocock SJ. Clinical trials: a practical approach. Chichester, UK: John Willey \& Sons; 2008.

2. Govaert TM, Thijs CT, Masurel N, Sprenger MJ, Dinant GJ, Knottnerus JA. The efficacy of influenza vaccination in elderly individuals. A randomized double-blind placebo-controlled trial. JAMA. 1994;272:1661-5

3. Statistics Netherlands, influenza related mortality per month [cited February 2018]. Available from: http://statline.cbs.nl.

4. Statistics Netherlands, population by age [cited February 2018]. Available from: http://statline.cbs.nl/ Statweb.

5. Simonsen L, Taylor RJ, Viboud C, Miller MA, Jackson LA. Mortality benefits of influenza vaccination in elderly people: an ongoing controversy. The Lancet Infectious diseases. 2007;7:658-66.

6. Darvishian M, Dijkstra F, van Doorn E, Bijlsma MJ, et al. Influenza Vaccine Effectiveness in the Netherlands from 2003/2004 through 2013/2014: The Importance of Circulating Influenza Virus Types and Subtypes. PLoS One. 2017;12:e0169528. 


Chapter 3

Influenza vaccination among Dutch general practitioners and their attitude towards influenza vaccination in the elderly 


\section{ABSTRACT}

Knowledge, attitudes and beliefs (KABs) towards influenza vaccination play a key role in healthcare workers' (HCWs') decisions to receive vaccination and can strongly influence patients' influenza vaccination uptake. We examined the knowledge, attitudes and beliefs of general practitioners (GPs) towards influenza vaccination, exploring their opinion on influenza vaccination in the elderly, mandatory HCW vaccination and the desirability of an influenza vaccination trial in the elderly with hospitalization and/or mortality as effect measure. From November 2018 to March 2019, surveys were emailed to GPs and GP-practices ( $n=1,676$ ) in three regions of the Netherlands. We assessed the self-reported influenza vaccination in GPs, reasons for (not) advising influenza vaccination to personnel, (not) supporting mandatory influenza vaccination for personnel and (not) desiring a trial on influenza vaccination in the elderly on hospitalization and/or mortality. Multivariable logistic regression models were used to determine predictors for GP influenza vaccination. A total of 552 surveys were completed and $71.9 \%$ of the GPs reported receiving influenza vaccination. Determinants for influenza vaccination in GPs were male sex ( $a O R$ 1.62, 95\% Cl 1.06-2.49, $p=0.03$ ) and age $\geq 60$ years (aOR 5.25, 95\% Cl 1.51-18.32, p = 0.01). Seventy-nine percent of the GPs recommend influenza vaccination for their practice personnel. Mandatory influenza vaccination for personnel was supported by $41.2 \%$ of the GPs with GP self-reporting influenza vaccination being the only determinant ( $a O R$ 10.03, 95\% Cl 5.69-17.70, $p=0.00$ ). An influenza vaccination trial on hospitalization and/or mortality was desired by $60.5 \%$ of the GPs. We concluded that the majority of Dutch GPs receives influenza vaccination and recommends influenza vaccination to their personnel. These high rates along with the hesitancy of GPs towards mandatory HCW influenza vaccination should be considered when policymakers decide on a mandate for influenza vaccination in HCW in general. 


\section{INTRODUCTION}

During an epidemic, influenza can affect all in society and elderly especially. Suddenly increased elderly care needs and absenteeism among caregivers and healthcare workers (HCWs) can disrupt the healthcare system. ${ }^{1,2}$ Besides vaccinating high-risk patients against influenza, also HCW vaccination is proposed to reduce its impact by preventing absenteeism and transmission to elderly and their caregivers. ${ }^{3}$ However, many HCWs choose to stay unvaccinated leaving coverage rates far below WHO-recommended levels. ${ }^{4}$

Knowledge, attitudes and beliefs (KABs) towards influenza vaccination play a key role in HCWs' decisions to receive vaccination and can strongly influence patients' influenza vaccination uptake..$^{5-7}$ Determinants of vaccine refusal in HCWs have been studied extensively. 8,9 However, most studies have been conducted in hospitals or elderly homes, whereas in many high-income countries like the Netherlands, general practitioners (GPs) are key actors in implementing influenza vaccination policy. Since previous findings cannot be translated to primary care, ${ }^{10}$ little remains known about the KAB of GPs towards influenza vaccination, especially in the largest target group; the elderly. New insights in KAB of GPs towards influenza vaccination could help developing interventions aimed at increasing vaccination uptake in the elderly and in primary healthcare personnel.

In the Netherlands, GPs have a long history as main implementers of the National Prevention Influenza Programme according to which high-risk groups are vaccinated against influenza free of charge. Given this background, the KAB of Dutch GPs towards influenza vaccination in HCWs and elderly is relevant. Moreover, their attitude towards currently debated topics regarding influenza vaccination - such as the desirability of (mandatory) HCW influenza vaccination ${ }^{11}$ and of a trial designed to evaluate the effect of influenza vaccination in the elderly on hospitalization and/or mortality ${ }^{12}$ - is important to consider for both policymakers and the scientific community when deciding on mandatory influenza vaccination or setting the agenda for new influenza vaccination related research.

Therefore, we examined (1) the KAB of Dutch GPs towards influenza vaccination, (2) evaluated demographic predictors for KAB, and (3) explored GPs' opinions on influenza vaccination in the elderly, mandatory HCW influenza vaccination and the desirability of an influenza vaccination trial on mortality, both before and during the influenza epidemic of 2018/2019. 


\section{METHODS}

Work-up

From November 2018 to March 2019, invitations to complete an online digital survey, were emailed to GPs and GP-practices in three regions (North, West and South) of the Netherlands. E-mail addresses were retrieved from databases provided by contact persons of the collaborating academic departments of Family Medicine in these regions.

To evaluate the effect of seasonality on survey results, GP contacts in the Southern-group were randomly divided into two groups, receiving requests to participate either before $(n=350)$ or during ( $n=337$ ) the influenza epidemic. To increase survey response, by the end of November 2018 electronic newsletters with a link to our survey were posted on GP platforms used in the Southern region. End of January, during the epidemic, e-mail requests were sent out to all other GP contacts; i.e. GPs in the Northern $(n=642)$ and Western region $(n=160)$ and the remaining GP contacts in the Southern region $(n=337)$. By chance, the academic department of Family Medicine in the Western region only held a small database of contacts. Therefore, in this region, we provided permission for participating GPs to distribute the survey to their regional GP colleagues.

Reminders were sent 1 and 2 weeks after sending the first invitation. The surveys were closed on January 2 and March 1. Fig.1 illustrates the start and end of the surveys in relation to the influenza-like illness (ILI) incidence.

\section{Questionnaire}

The survey consisted of 14 closed-ended questions mostly using 5-point Likert scales (Supplementary information). We adopted some of the questions of a survey of the influenza vaccination rate among Dutch GPs in 2008, so that we could compare reported influenza vaccination rates among Dutch GPs over time. ${ }^{14}$ When applicable, the Checklist for Reporting Results of Internet E-Surveys (CHERRIES) statement was followed. ${ }^{15}$

Draft questionnaires were previously reviewed by ten university staff members specialized in primary care research and adjusted accordingly. The medical ethics committee of the Maastricht University Medical Center confirmed that the Medical Research Involving Human Subjects Act did not apply since no patients were included. To avoid any effect on the GPs' vaccination behavior, surveys were sent out only 2 weeks after the influenza vaccination campaign had ended (Fig.1).

\section{Outcome measures}

Outcome measures were the self-reported influenza vaccination coverage in GPs and the assessment of determinants and reasons for (not) being vaccinated against influenza, (not) advising (mandatory) HCW vaccination, (not) supporting the current influenza vaccination policy and (absence of) desirability of a trial on influenza vaccination in the elderly evaluating hospitalization and/or mortality as an endpoint. 


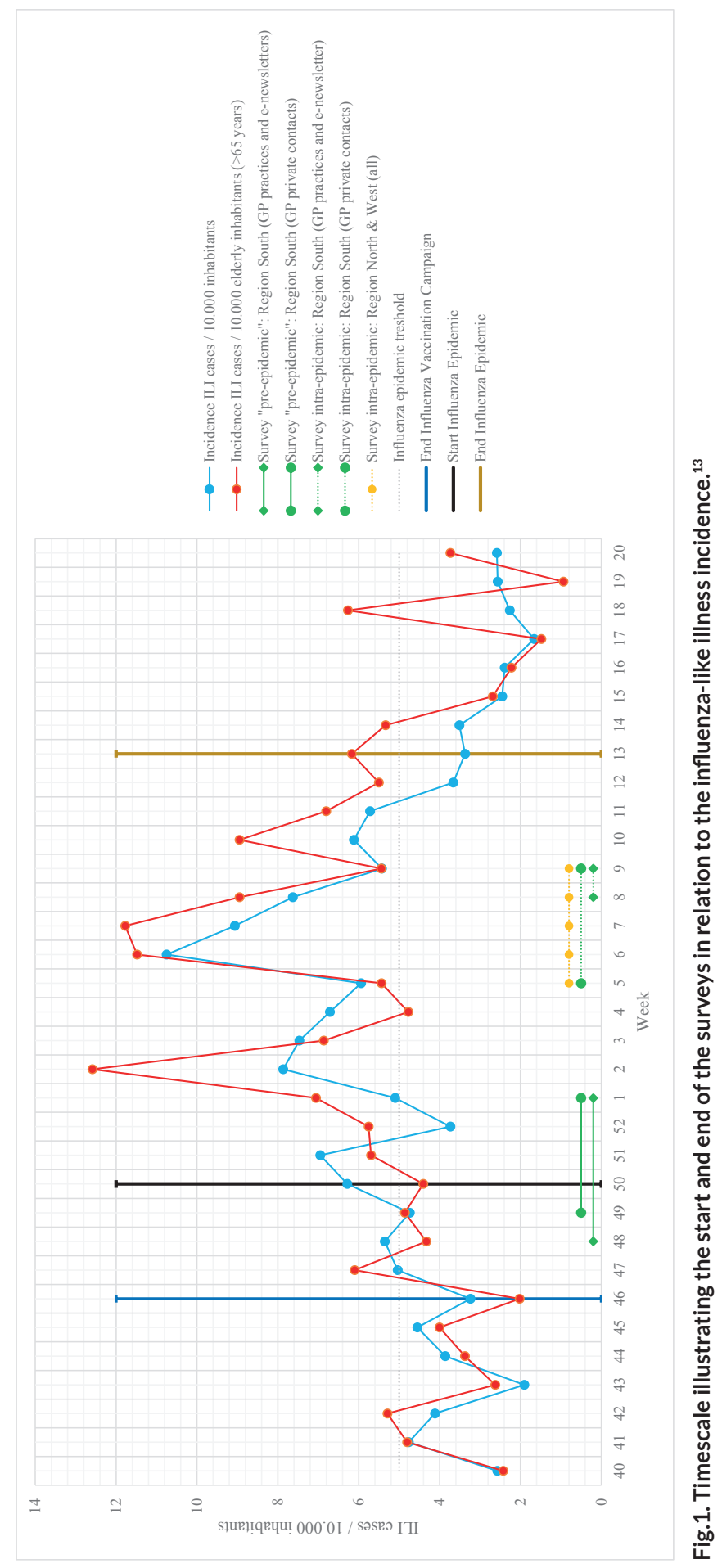


Analysis

Only fully completed forms were used for analyses. After testing for multicollinearity, all demographic variables (by all means: age, gender, working experience, part-time/full-time work, practice form) were entered in an ordinal logistic regression model to calculate the adjusted odds ratio (aOR). If assumptions regarding goodness of fit and/or proportional odds assumption were violated, dependent variables with Likert-scale (five parameters) were recoded into three parameter variables and assumptions were retested. If assumptions were still not met, outcome variables were reduced to two levels to perform binary logistic regression. We used the chisquare test to test for any demographic differences between the GPs per region and per time frame (pre or intra-epidemically).When comparing continuous data, the Shapiro-Wilk test was used to test for normality. If not normally distributed, non-parametric tests (Mann-Whitney Test) were used.

\section{RESULTS}

\section{Demographics}

A total of 1,676 GPs or GP-practices were directly asked to participate. Since the survey was also accessible by electronic newsletter, an unknown number of potential participants was indirectly reached. Eventually, 601 GPs participated in completing 552 surveys. Since the denominator was not known exactly, the total response rate is estimated as being $\leq 32.9 \%$ (552/1,676). Accurate response rates were calculated from the survey groups including GPS who were only addressed personally, varying between 29.1\% and 39.5\% (mean 33.6\%). The basic characteristics of responders and national figures are presented in Table 1. Demographics of those participating in the Southern region before and during the epidemic did not differ significantly (results not shown).

Survey results

Survey results are shown in Tables 2-4. In the 2018/2019 season, 397 (71.9\%) of the GPS reported receiving influenza vaccination. Most important reasons were reducing the risk of getting influenza (61.2\%) and transmitting influenza to patients (87.7\%). Most important reasons for refusing influenza vaccination were not belonging to a risk group (51.0\%) and the conviction that one was already protected against influenza (25.2\%) (Table 3). Among 'other motives' uncertainty regarding the usefulness of the vaccine was most frequently mentioned (13/41). Regression analysis showed two determinants for self-reported influenza vaccination among GPs: male sex (aOR 1.62, 95\% Cl 1.06-2.49, $\mathrm{p}=0.03$ ) and age $\geq 60$ years (aOR 5.25, $95 \% \mathrm{Cl} 1.51-18.32, \mathrm{p}=0.01$ ). Reasons for supporting the influenza vaccination policy (or not) can be found in Table 4. 
Table 1. Basic characteristics of respondents compared with national figures.

\begin{tabular}{|c|c|c|}
\hline & Respondents ( $n=552)$ No. (\%) & All Dutch GPs $(N=9,898)$ \\
\hline \multicolumn{3}{|l|}{ Sex } \\
\hline Male & $300(54.3 \%)$ & $47 \%$ \\
\hline Female & $252(45.7 \%)$ & $53 \%$ \\
\hline \multicolumn{3}{|l|}{ Age groups } \\
\hline$\leq 40$ years & $91(16.5 \%)$ & $25.9 \%$ \\
\hline 41-49 years & $153(27.7 \%)$ & $29.7 \%$ \\
\hline $50-59$ years & $187(33.9 \%)$ & $28.7 \%$ \\
\hline$\geq 60$ years & $121(21.9 \%)$ & $15.7 \%$ \\
\hline \multicolumn{3}{|l|}{ Work experience as a GP } \\
\hline$\leq 10$ years & $114(20.7 \%)$ & - \\
\hline 11-19 years & $183(33.2 \%)$ & - \\
\hline$\geq 20$ years & 255 (46.2\%) & - \\
\hline \multicolumn{3}{|l|}{ Employment } \\
\hline$\leq 0.6 \mathrm{fte}$ & $136(24.6 \%)$ & $19.4 \%$ \\
\hline$>0.6 \mathrm{fte}$ & $416(75.4 \%)$ & $80.6 \%$ \\
\hline \multicolumn{3}{|l|}{ Working situation } \\
\hline In a solo practice & $117(21.2 \%)$ & $17.0 \%$ \\
\hline In a non-solo practice & $392(71.0 \%)$ & $83.1 \%$ \\
\hline As a locum & $43(7.8 \%)$ & * \\
\hline
\end{tabular}

GP: general practitioner, fte: full-time equivalent.

*Data unavailable; GPs working as a locum have been assigned to either solo or non-solo practices based on the practice they work for (solo or non-solo).

Mandatory influenza vaccination in practice personnel

Of all responding GPs, 436 (79.0\%) recommend influenza vaccination for their practice personnel (Table 2.2). Mandatory influenza vaccination of personnel is supported by $41.2 \%$ and rejected by $36.9 \%$ of the GPs (Table 2.1). Reported influenza vaccination in GPs was a predictor for supporting mandatory influenza vaccination in practice personnel (aOR 10.03, 95\% Cl 5.69-17.70, $p=0.00)$.

Estimated vaccine efficacy and vaccination rate

Median estimated efficacy of influenza vaccination for preventing influenza in the elderly was 60\% (range 0-100\%) and significantly higher in those who received influenza vaccination (60\%, range $1-95 \%$ ) than those not receiving influenza vaccination ( $50 \%$, range $0-100 \%, p=0.00$ ). The median estimated vaccination rate of risk groups within the GP practices was $60 \%$ (range 0-95\%) and did not differ between vaccinated and unvaccinated GPs (median 60\%, range $0-95 \%$ vs. 60\%, range 3-90\%; $p=0.44)$. 
Desirability of a placebo-controlled influenza vaccination trial on hospitalization / mortality

Of all GPs, $60.5 \%$ desires a trial evaluating the effect of influenza vaccination in the elderly on hospitalization and/or mortality. GPs not receiving influenza vaccination desired such a trial more frequently than those receiving influenza vaccination (68.4\% vs. 57.4\%, $p=0.02$.

\section{Seasonal and regional differences}

No significant differences in survey results were demonstrated between the three regions (results not shown), except for the estimated vaccination rate which was significantly lower in the Western-group compared to the Southern and Northern regions; 50\% (range 20-75\%) vs. $60 \%$ (range 0-95\%), p = 0.00. Pre-epidemically 36.6\% of the GPs supported mandatory influenza vaccination in HCWs, increasing to 48.0\% intra-epidemically (overall 41.2\%). The unadjusted OR of the intra-epidemic season vs. the pre-epidemic season on support of an influenza vaccination mandate (agree vs. neutral or disagree) was 1.60 (95\% Cl 1.03-2.48, p = 0.04). After correcting for demographic variables we found an aOR of 1.49 (95\% Cl 0.94- 2.38, p = 0.09).

\section{Table 2.1-2.3. Response to the survey questions.}

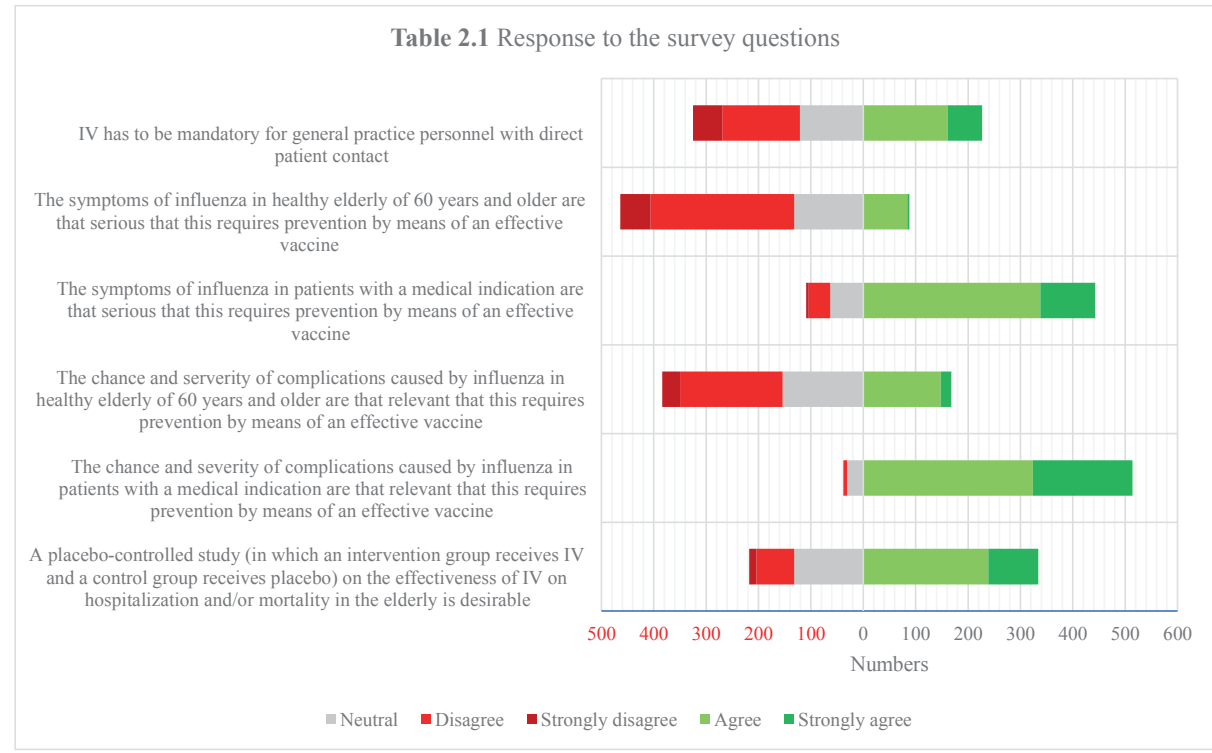




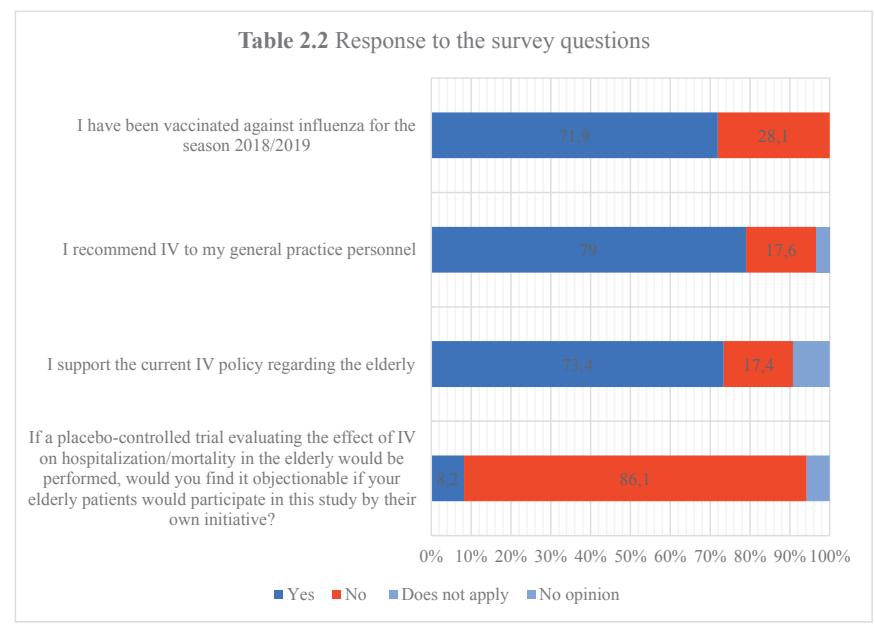

Table 2.3 Response to the survey questions

Do you agree with the age criterion ( $>60$ years) as an indication for influenza vaccination?

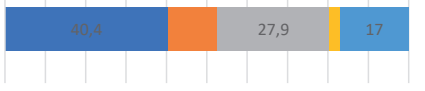

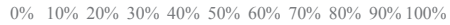

- Yes

No opinion

No, the age criterion has to be higher

No, the age criterion has to be lower

- No, I think age is not a correct indication for influenza vaccination 


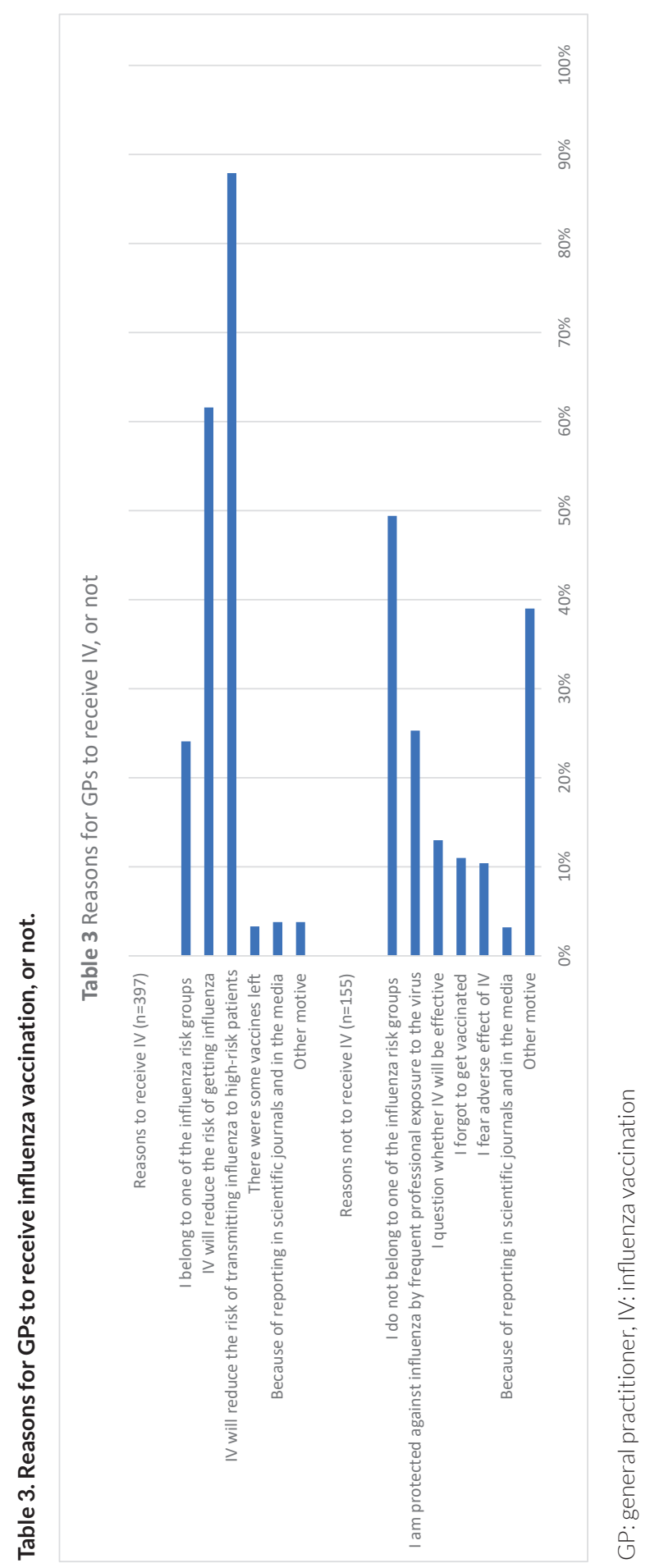




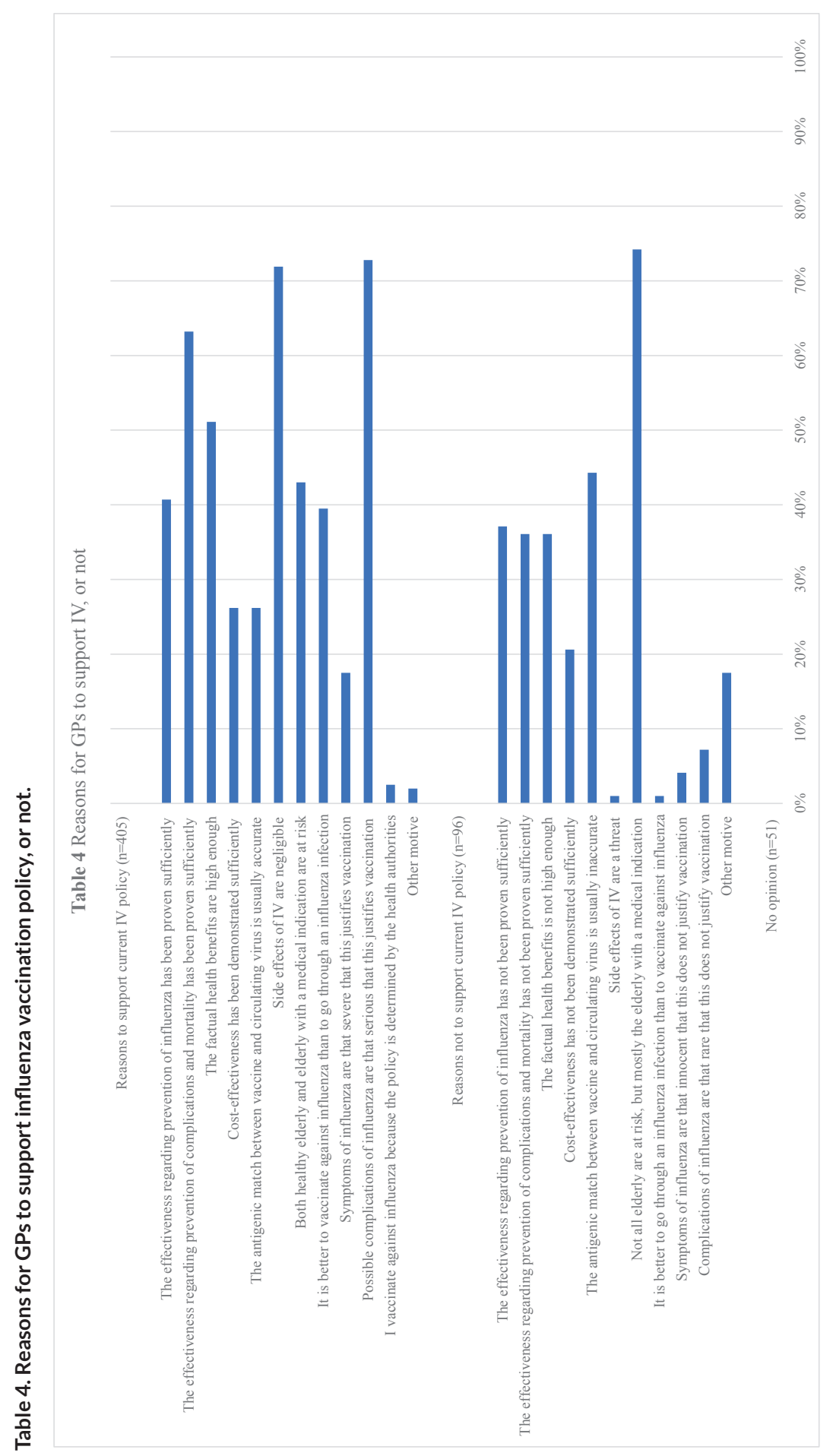




\section{DISCUSSION}

We examined the knowledge, attitudes and beliefs of general practitioners towards influenza vaccination in different regions in the Netherlands before and during the 2018/2019 influenza epidemic. Our study showed that the majority of GPs receives influenza vaccination (71.9\%), recommends influenza vaccination for their practice personnel (79.0\%) and desires for a trial evaluating the effect of influenza vaccination on hospitalization and/or mortality in the elderly (60.5\%). Male sex (aOR 1.62) and age $\geq 60$ years (aOR 5.25) were significant determinants for selfreported influenza vaccination among GPs. Reported influenza vaccination in GPs was a strong predictor for supporting mandatory influenza vaccination in practice personnel (aOR 10.03).

Strengths of our study are the generalizability since we conducted our study in different regions. In the Netherlands, GPs have always been key actors in implementing influenza vaccination. Given their experience and expertise, the attitude of Dutch GPs towards this topic is particularly interesting. Our methodological approach also allowed us to compare survey responses before and during the influenza season which has not been done before. Also - by adopting some questions of a previous study ${ }^{14}$ - we could compare GP vaccination rates over time. Finally, this study evaluates currently debated topics regarding influenza vaccination (i.e. mandatory HCW influenza vaccination and desirability of an influenza vaccination trial on mortality) that have not been evaluated in such large populations of GPs before.

Some limitations of this study should be discussed. Although the response rate of approximately $30 \%$ is fair in this field, ${ }^{16}$ and attempts to increase questionnaire response by using online platforms were made, selective response cannot be excluded. Also, since the participating GPs were affiliated to an academic department of Family Medicine, this could negatively affect the representativeness of our respondents. However, the basic characteristics of our respondents were roughly comparable to national statistics. Also, participating GPs did not seem to have a preoccupation towards influenza vaccination since their median estimated vaccination coverage rates and estimated vaccine efficacy rates correspond well to the national statistics (49.9\% in risk groups) ${ }^{17}$ and literature (vaccine efficacy 50-60\%) ${ }^{18}$ respectively. For these reasons and given their affiliation to different departments in different regions in the Netherlands, we consider our study population as representative. Finally, post-hoc sample size calculation (not presented) showed that at least 369 participants needed to be included in order to have an acceptable 95\% Cl around a proportion of 0.6 of GPs being vaccinated. Thus, responses $(n=552)$ can be considered high enough to draw conclusions based on these findings.

Because influenza vaccination in GPs was self-reported, social desirability bias cannot be excluded. However, research shows that questionnaire data overestimates vaccination rates by less than 10\%,20 limiting the potential effect of such bias. Finally, for methodological reasons previously explained, we were still enrolling participants in our 'pre-epidemic' group when the epidemic had already started. But by that time ILI-incidence was still low, and the incidence of 
ILI in the elderly - which has our special interest - started to increase later, coinciding with our second (intra-epidemic) survey rounds (Fig.1). Therefore, we think our surveys still discriminate between the 'pre-epidemic' and intra-epidemic season.

Coverage rate, motives and predictors of influenza vaccination in GPS

Influenza vaccination coverage among European HCWs is generally below 30\%, ${ }^{20}$ and has been showing declining trends. ${ }^{21}$ Vaccination rates among GPS vary widely; ranging from $12 \%$ in Slovenia ${ }^{22}$ to over $75 \%$ in some Italian regions. ${ }^{23}$ Most recent statistics on Dutch GP's influenza vaccination coverage date back from the pandemic 2009/2010 season, reporting a (seasonal) influenza vaccination coverage rate of $63 \% .{ }^{24}$ Especially when compared to the latest non pandemic rates of 36\% for seasonal influenza vaccination in 2007/2008, ${ }^{14}$ the coverage rate of $71.9 \%$ found in our study suggests a relevant increase. For reasons previously explained, this increase is not likely to be explained by selection bias. A potential explanation for the increase could be the gradual implementation of the recommendations for $\mathrm{HCW}$ to be vaccinated against influenza that were introduced in 2007.25

Besides age $>60$ years (which is an indication for influenza vaccination), male sex was a predictor for vaccination uptake in GPs, which is consistent with previous studies among GPs. ${ }^{14}$, ${ }^{24}$ In our study, these differences in vaccination coverage between sex were consistent over all age groups, whereas in the general (non-GP) population females tend to be vaccinated more often. ${ }^{26}$ This is suggested to be related to the greater propensity of women for seeking health care. ${ }^{27}$ Although this effect is likely to be smaller in healthcare professionals, our study did not yield supportive data to explain for the inverse relation that we found.

In our study, most frequently reported motives in GPs for receiving influenza vaccination were personal protection against influenza and lowering of the risk of transmitting influenza to patients, whereas the most common reasons for not being vaccinated were: having no medical indication for influenza vaccination and the conviction that one is already protected against influenza. These motives do not seem to have changed over the past decade ${ }^{14}$ and apply internationally. ${ }^{9}$ Similar to our study, less frequently reported motives (ranging from $2.5 \%$ to $28.6 \%$ ) for non-vaccination in GPs are: concerns about side effects, forgetfulness, and doubts about the vaccine's efficacy. ${ }^{9}$ Besides all quantitative data, qualitative data showed that also the belief that influenza is not a serious illness is a potential barrier for HCW vaccination. ${ }^{8}$ Our study clarifies that - unlike in patients with a medical indication - the majority of GPS do not agree that the seriousness of symptoms and the severity and chance of complications of influenza in healthy elderly are that relevant that this requires prevention by means of an effective vaccine. 
Attitude towards mandatory influenza vaccination in HCW and towards a new trial

To our best knowledge, this is the first study to examine the attitudes of GPs towards mandatory influenza vaccination in practice personnel. Whereas only $41.2 \%$ of the GPs in our study supports mandatory vaccination of practice personnel with direct patient contact, Desante et al. showed that in the United States, $84 \%$ of the physicians working in internal medicine or emergency medicine supported mandatory influenza vaccination among $\mathrm{HCW}^{26}$ Differences in the severity of complications caused by influenza in patients presenting in this setting compared to primary care may partly explain for these differences.

The efficacy of influenza vaccination in the elderly has long been a topic of debate, mainly due to the lack of direct RCT-based evidence on effects of influenza vaccination on severe morbidity and mortality in the elderly. This study is the first now to report an uninformed opinion of a majority (60.5\%) of GPs desiring such a trial. By uninformed, we mean the lack of explicit expertise on influenza vaccination and the ethical or methodological aspects involved in conducting research evaluating the effects of influenza vaccination.

Regional and seasonal influence on the attitude of GPs towards influenza vaccination in the elderly Regional variations in GPs' perceptions and practices regarding vaccination have been observed in France. ${ }^{29}$ However, this study did not focus on influenza vaccination explicitly. Besides the estimated coverage rates of influenza vaccination varying up to $12 \%$ per region, we did not find regional differences in attitudes of GPs towards influenza vaccination. This could be explained by close adherence to Dutch medical guidelines.

Up to our knowledge, no studies quantified the effect of seasonality on attitudes of GPS on influenza vaccination before. A qualitative Australian study did suggest that the severity of the previous influenza season could affect the attitude of stakeholders towards mandatory influenza vaccination in HCW one year later. ${ }^{30}$ Although we examined this effect within the same season, we could not demonstrate a significant effect of the epidemic occurrence of influenza on the GPs' attitude towards mandatory influenza vaccination in HCW. It should be noted that the 2018/2019 influenza season in the Netherlands was relatively mild, potentially limiting the near significant effect (aOR 1.49, 95\% Cl 0.94-2.38).

Implications and novelty of our study

Our study found a high influenza vaccination coverage rate in GPs (71.9\%), indicating a relevant increase over the past decade. ${ }^{14}$ In the absence of surveillance systems monitoring influenza vaccination coverage rates in primary care on any (national or European) level and considering the present-day debate on introducing mandatory influenza vaccination for HCWs, our upto-date information on GP coverage rates and their attitudes towards influenza vaccination is relevant for both policymakers and researchers. In this study, we did not evaluate vaccination rates in HCWs other than GPs. Previous studies learned that coverage rates in other HCWs are generally lower than in physicians (ranging between 20\% and 40\%). ${ }^{31}$ We do know that 
physicians' encouragement to get vaccinated is an important factor in HCW vaccine uptake. ${ }^{32}$, ${ }^{33}$ Considering that the majority of GPs is vaccinated and recommends influenza vaccination to their practice personnel, we expect that this translates into high vaccination rates in practice personnel as well, although other HCWs than GPs were not included in this study. The high vaccination coverage in GPs as such, the hesitancy of GPs to mandate influenza vaccination in HCWs, the fact that non-institutionalized elderly are easily exposed to many potential vectors of influenza other than HCWs, but also the fact that GPs also regularly visit patients that may be very ill and institutionalized, should all be taken into account when policymakers decide on mandatory influenza vaccination for HCWs in general and - more explicitly - in primary care.

This is the first study to objectify the effect of seasonality on attitudes of GPs on IV. Our finding that GPs' attitudes towards mandatory influenza vaccination in HCW does not substantially change during the influenza season should be interpreted with care, as explained previously. In case stakeholders or HCWs are involved in deciding on such a mandate, policymakers should be aware that, especially during a severe influenza epidemic, a potential seasonal effect on this opinion, cannot be ruled out.

A novel finding is that $60.5 \%$ of the GPs' desires a placebo-controlled trial on influenza vaccination in the elderly evaluating mortality as an endpoint and that $86.1 \%$ would have no objections if their elderly patients would participate in such a trial, exposing them to the harmful risks of influenza. This (uninformed) opinion has no direct implications for the justification and feasibility of such a trial. However, it should be seen as an important sign for the scientific community to clearly communicate on the state of evidence on the effects of influenza vaccination on morbidity and mortality ${ }^{34}$ in the elderly and the substantial ethical, methodological and practical barriers to be addressed, ${ }^{12}$ before a placebo-controlled trial with severe complications and mortality as outcomes would be justified.

Finally, if new research in the field is conducted, this study provides new insights in knowledge gaps or outstanding questions that could be addressed. For instance, the observed uncertainty among GPs in our study on the need for influenza vaccination in 'healthy' elderly. 


\section{CONCLUSION}

Whereas the positive behavior towards influenza vaccination is reflected by the majority of GPs receiving influenza vaccination and recommending influenza vaccination to their practice personnel, the GPs' attitudes towards mandatory influenza vaccination and the beneficial effects of influenza vaccination in the healthy elderly on severe morbidity and mortality should be considered hesitant. Given the majority of GPs that like to see a trial being conducted on hospitalization and/or mortality as an endpoint, and the identified ethical and methodological barriers for conducting such a trial, it is important to explicitly discuss the current state of evidence on the effects of influenza vaccination and these barriers with the GP community. Our findings should be taken into consideration when policymakers decide on a mandate for influenza vaccination and when researchers set up the agenda for influenza-related research or communicate on their findings. 


\section{REFERENCES}

1. European Centre for Disease Prevention and Control. Factsheet about seasonal influenza [cited October 2019]. Available from: https://ecdc.europa.eu/en/seasonal-influenza/facts/factsheet.

2. European Commission. Importance of HCW vaccination [cited October 2019]. Availavle from: https:// ec.europa.eu/health/vaccination/influenza_en.

3. Maltezou HC, Poland GA. Vaccination policies for healthcare workers in Europe. Vaccine 2014;32:487680.

4. Wicker S, Marckmann G. Vaccination of health care workers against influenza: is it time to think about a mandatory policy in Europe? Vaccine 2014;32:4844-8.

5. Martinez-Baz I, Aguilar I, Moran J, Albeniz E, Aldaz P, Castilla J. Factors associated with continued adherence to influenza vaccination in the elderly. Preventive medicine 2012;55:246-50.

6. Bovier PA, Chamot E, Bouvier Gallacchi M, Loutan L. Importance of patients' perceptions and general practitioners' recommendations in understanding missed opportunities for immunisations in Swiss adults. Vaccine 2001;19:4760-7.

7. Chiatti C, Barbadoro P, Lamura G, et al. Influenza vaccine uptake among community-dwelling Italian elderly: results from a large cross-sectional study. BMC public health 2011;11:207.

8. Lorenc T, Marshall D, Wright K, Sutcliffe K, Sowden A. Seasonal influenza vaccination of healthcare workers: systematic review of qualitative evidence. BMC health services research 2017;17:732.

9. Collange F, Verger P, Launay O, Pulcini C. Knowledge, attitudes, beliefs and behaviors of general practitioners/family physicians toward their own vaccination: A systematic review. Human vaccines \& immunotherapeutics 2016;12:1282-92.

10. Ridda I, Lindley IR, Gao Z, McIntyre P, Macintyre CR. Differences in attitudes, beliefs and knowledge of hospital health care workers and community doctors to vaccination of older people. Vaccine 2008;26:5633-40.

11. Ministry of Health, Welfare and Sport. Letter of State Secretary Paul Blokhuis on evaluation of mandatory influenza vaccination in HCW. [Kamerbrief Blokhuis over Maatregelen Griep] [cited October 2019]. Available from: https://www.rijksoverheid.nl/ministeries/ministerie-van-volksgezondheid-welzijn-ensport/documenten/kamerstukken/2018/10/10/kamerbrief-over-maatregelen-griep.

12. Verhees RAF, Dondorp W, Thijs C, Dinant GJ, Knottnerus JA. Influenza vaccination in the elderly: Is a trial on mortality ethically acceptable? Vaccine 2018;36:2991-7.

13. Nivel. Incidence of influenza-like illness during the 2018/2019 influenza season [cited October 2019]. Available from: https://www.nivel.nl/nl/zorgregistraties-eerste-lijn/surveillance.

14. Opstelten W, van Essen GA, Ballieux MJ, Goudswaard AN. Influenza immunization of Dutch general practitioners: vaccination rate and attitudes towards vaccination. Vaccine 2008;26:5918-21.

15. Eysenbach G. Improving the quality of Web surveys: the Checklist for Reporting Results of Internet E-Surveys (CHERRIES). Journal of medical Internet research 2004;6:e34.

16. Morton SM, Bandara DK, Robinson EM, Carr PE. In the 21st Century, what is an acceptable response rate? Australian and New Zealand journal of public health 2012;36:106-8.

17. Nivel. Report on vaccination coverage rates in the Netherlands [cited October 2019]. Available from: https://www.nivel.nl/nl/publicatie/monitor-vaccinatiegraad-nationaal-programmagrieppreventie-2017.

18. Govaert TM, Thijs CT, Masurel N, Sprenger MJ, Dinant GJ, Knottnerus JA. The efficacy of influenza vaccination in elderly individuals. A randomized double-blind placebo-controlled trial. Jama 1994;272:1661-5. 
19. Brien S, Kwong JC, Buckeridge DL. The determinants of 2009 pandemic A/H1N1 influenza vaccination: a systematic review. Vaccine 2012;30:1255-64.

20. Mereckiene J, Cotter S, Nicoll A, et al. Seasonal influenza immunisation in Europe. Overview of recommendations and vaccination coverage for three seasons: pre-pandemic (2008/09), pandemic (2009/10) and post-pandemic (2010/11). Euro surveillance : bulletin Europeen sur les maladies transmissibles = European communicable disease bulletin 2014;19:20780.

21. Dini G, Toletone A, Sticchi L, Orsi A, Bragazzi NL, Durando P. Influenza vaccination in healthcare workers: A comprehensive critical appraisal of the literature. Human vaccines \& immunotherapeutics 2018;14:772-89.

22. Petek D, Kamnik-Jug K. Motivators and barriers to vaccination of health professionals against seasonal influenza in primary healthcare. BMC health services research 2018; 18:853.

23. Desiante F, Caputi G, Cipriani R, et al. Assessment of coverage and analysis of the determinants of adherence to influenza vaccination in the general practitioners of Taranto. Annali di igiene : medicina preventiva e di comunita 2017;29:256-63.

24. Opstelten W, van Essen GA, Heijnen ML, Ballieux MJ, Goudswaard AN. High vaccination rates for seasonal and pandemic (A/H1N1) influenza among healthcare workers in Dutch general practice. Vaccine 2010;28:6164-8.

25. Health Council of the Netherlands. Influenza vaccination: revision of the indication [cited January 2020]. Available from: https://www.healthcouncil.nl/documents/advisory-reports/2007/03/08/ influenza-vaccination-revision-of-the-indication.

26. Centers for Disease Control and Prevention. Influenza vaccination coverage rates by sex [cited January 2020]. Available from: https://www.cdc.gov/flu/fluvaxview/coverage-1617estimates.htm\#by-sex.

27. Chambers C, Skowronski DM, Rose C, et al. Should Sex Be Considered an Effect Modifier in the Evaluation of Influenza Vaccine Effectiveness? Open forum infectious diseases 2018;5:ofy211.

28. deSante JE, Caplan A, Shofer F, Behrman AJ. Physician attitudes towards influenza immunization and vaccine mandates. Vaccine 2010;28:2517-21.

29. Collange F, Zaytseva A, Pulcini C, Bocquier A, Verger P. Unexplained variations in general practitioners' perceptions and practices regarding vaccination in France. European journal of public health 2019;29:2-8.

30. Moran A, Agaliotis M, Seale H. The views of key stakeholders around mandatory influenza vaccination of hospital and aged care staff: Examining the current climate in Australia. Vaccine 2019;37:705-10.

31. Abramson ZH, Levi O. Influenzavaccination among primary healthcareworkers. Vaccine 2008;26:24829.

32. Corace K, Prematunge C, McCarthy A, Nair RC, Roth V, Hayes T, et al. Predicting influenza vaccination uptake among health care workers: what are the key motivators? American journal of infection control 2013;41:679-84.

33. Boey L, Bral C, Roelants M, De Schryver A, Godderis L, Hoppenbrouwers K, et al. Attitudes, believes, determinants and organisational barriers behind the low seasonal influenza vaccination uptake in healthcare workers - A cross-sectional survey. Vaccine 2018;36:3351-8.

34. Armstrong BG, Mangtani P, Fletcher A, Kovats S, McMichael A, Pattenden S, et al. Effect of influenza vaccination on excess deaths occurring during periods of high circulation of influenza: cohort study in elderly people. BMJ (Clinical research ed) 2004;329:660. 


\section{SUPPLEMENTARY INFORMATION}

\section{Survey questions}

1 I have been vaccinated against influenza for the season 2018/2019.

$\square \quad$ Yes (continue to question 2a)

$\square \quad$ No (continue to question $2 \mathrm{~b}$ )

2a I have been vaccinated for the following reason(s): (Multiple answers possible)

$\square \quad$ I belong to one of the influenza risk groups (by age criterion and/or by medical indication)

$\square \quad$ Vaccination will reduce the risk of getting influenza

$\square$ Vaccination will reduce the risk of transmitting influenza to high-risk patients

$\square \quad$ There were some vaccines left

$\square \quad$ Because of reporting in scientific journals and in the media

$\square \quad$ Other motive...

2b I have not been vaccinated for the following reason(s): (Multiple answers possible)

$\square \quad$ I do not belong to one of the influenza risk groups (neither by age criterion nor by medical indication)

$\square \quad$ I am protected against influenza by frequent professional exposure to the virus

$\square \quad$ I question whether in my case vaccination will be effective

$\square \quad$ I forgot to get vaccinated

$\square \quad$ I fear side effects of influenza vaccination

$\square \quad$ Because of reporting in scientific journals and in the media

$\square \quad$ Other motive...

3 I recommend influenza vaccination to my general practice personnel.

$\square$ Yes

$\square \quad$ No

$\square \quad$ Does not apply; I do not have any personnel

4 Influenza vaccination should become mandatory for general practice personnel with direct patient contact.

$\square$ Strongly disagree $\square$ Disagree $\square$ Neutral $\square$ Agree $\square \quad$ Strongly agree 
5 Do you agree with age criterion (>60 years) as an indication for influenza vaccination?

$\square \quad$ Yes, I do

$\square \quad$ No, the age criterion has to be higher

$\square \quad$ No, the age criterion has to be lower

$\square \quad$ No, I think age is not a correct indication for influenza vaccination

$\square \quad$ No opinion

6 How would you estimate the effectiveness of influenza vaccination in the elderly on preventing influenza in the elderly, in case of good antigenic match between the vaccine and the circulating influenza viruses?

- ...\%

7 How would you estimate the vaccination rate of your risk patients in the season 2018/2019?

- $\quad \ldots \%$

8 The symptoms of influenza in healthy elderly of 60 years and older are that serious that this requires prevention by means of an effective vaccine.

$\square$ Strongly disagree $\square$ Disagree $\square$ Neutral $\square$ Agree $\square$ Strongly agree

9 The symptoms of influenza in patients with a medical indication are that serious that this requires prevention by means of an effective vaccine.

$\square$ Strongly disagree $\square$ Disagree $\square$ Neutral $\square$ Agree $\square$ Strongly agree

10 The chance and severity of complications caused by influenza in healthy elderly of 60 years and older are that relevant that this requires prevention by means of an effective vaccine.

$\square$ Strongly disagree $\square$ Disagree $\square$ Neutral $\square$ Agree $\square$ Strongly agree

11 The chance and severity of complications caused by influenza in patients with a medical indication are that relevant that this requires prevention by means of an effective vaccine.

Strongly disagree $\square$ Disagree $\square$ Neutral $\square$ Agree $\square$ Strongly agree

12 I support the current influenza vaccination policy regarding the elderly.

$\square \quad$ Yes

$\square \quad$ No

$\square \quad$ No opinion 
Why DO you support the current policy regarding the elderly? (Multiple answers possible)

$\square \quad$ The effectiveness regarding prevention of influenza has been proven sufficiently

$\square \quad$ The effectiveness regarding prevention of complications and mortality has been proven sufficiently

$\square \quad$ The health benefits are high enough

$\square \quad$ Cost-effectiveness has been demonstrated sufficiently

$\square \quad$ The antigenic match between vaccine and circulating virus is usually accurate

$\square \quad$ Side effects of influenza vaccination are negligible

$\square \quad$ Both healthy elderly and elderly with a medical indication are at risk

$\square \quad$ It is better to vaccinate against influenza than to go through an influenza infection

$\square$ Symptoms of influenza are that severe that this justifies vaccination

$\square$ Possible complications of influenza are that serious that this justifies vaccination

$\square \quad$ I vaccinate against influenza because the policy is determined by the health authorities, but personally I do not support this policy

$\square \quad$ Other motive...

Why DO you NOT support the current policy regarding the elderly? (Multiple answers possible)

$\square \quad$ The effectiveness regarding prevention of influenza has not been proven sufficiently

$\square \quad$ The effectiveness regarding prevention of complications and mortality has not been proven sufficiently

$\square \quad$ The factual health benefits are not high enough

$\square$ Cost-effectiveness has not been demonstrated sufficiently

$\square \quad$ The antigenic match between vaccine and circulating virus is usually inaccurate

$\square \quad$ Side effects of influenza vaccination are a threat

$\square \quad$ Not all elderly are at risk, but mostly the elderly with a medical indication

$\square \quad$ It is better to go through an influenza infection than to vaccinate against influenza

$\square$ Symptoms of influenza are that innocent that this does not justify vaccination

$\square$ Complications of influenza are that rare that this does not justify vaccination

$\square \quad$ Other motive...

13 A placebo-controlled study (in which the intervention group receives the influenza vaccine and the control group receives placebo) on the effectiveness of influenza vaccination on hospitalization and/or mortality in the elderly is desirable.

$\square$ Strongly disagree $\square$ Disagree $\square$ Neutral $\square$ Agree $\square$ Strongly agree 
14 The desirability of a placebo-controlled trial on influenza vaccination in the elderly is currently debated. If such a trial would be performed, would you find it objectionable if your elderly patients would participate in this study by their own initiative?

$\square \quad$ Yes

$\square \quad$ No

$\square \quad$ No opinion

Gender

$\square \quad$ Male

$\square \quad$ Female

Age

- $\quad$.......years old

For how long have you been working as a practicing GP?

- .......years

How many days per week do you work as a practicing GP?

$\square \quad$ Three or less days per week

$\square \quad$ Four or more days per week

Practice form

$\square$ Solo

$\square \quad$ Non-solo (duo/group)

$\square \quad$ I work as a locum

Remarks 




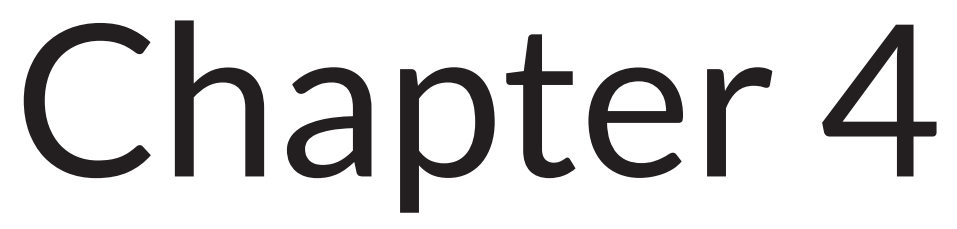

\section{Influenza vaccination in the elderly: mortality after 25 years follow-up of a trial}

Ruud A.F. Verhees

Carel Thijs

Ton Ambergen

Geert-Jan Dinant

Johannes A. Knottnerus

PloS one 2019;14:e0216983

Huisarts Wet 2020;63:16-9 


\section{ABSTRACT}

Influenza vaccination is proven effective in preventing influenza. However, long-term effects on mortality have never been supported by direct evidence. In this study we assessed the long-term outcome of influenza vaccination on mortality in the elderly by conducting a 25 -year follow-up study of an RCT on the efficacy of influenza vaccination as baseline. The RCT had been conducted in the Netherlands five years before vaccination was recommended for those aged $\geq 65$ and 17 years before recommending it for those aged $\geq 60$. The RCT included 1,838 community-dwelling elderly aged $\geq 60$ that had received an intramuscular injection with the inactivated quadrivalent influenza vaccine ( $n=927$ ) or placebo ( $n=911)$ during the $1991 / 1992$ winter. In our follow-up study, outcomes included all-cause mortality, influenza-related mortality and seasonal mortality. Unadjusted and adjusted hazard ratios (HRs) were estimated by Cox regression and sub-hazard ratios (SHRs) by competing risk models. Secondary analyses included subgroup analyses by age and disease status. The vital status up to January 1, 2017 was provided in 1,800/1,838 (98\%) of the cases. Single influenza vaccination did not reduce all-cause mortality when compared to placebo (adjusted HR 0.95, 95\% Cl 0.85-1.05). Also, no differences between vaccination and placebo group were shown for underlying causes of death or seasonal mortality. In those aged 60-64, median survival increased with 20.1 months (95\% Cl 2.4-37.9), although no effects on all-cause mortality (adjusted HR 0.86, 95\% Cl 0.72-1.03) could be demonstrated in survival analysis. In conclusion, this study did not demonstrate a statistically significant effect following single influenza vaccination on long-term mortality in community-dwelling elderly in general. We propose researchers designing future studies on influenza vaccination in the elderly to fit these studies for longer-term follow-up, and suggest age group comparisons in observational research. 


\section{INTRODUCTION}

Influenza has a major impact on morbidity and mortality, especially among the elderly. In the United States, influenza accounts for about 32,000 (5\%) of 600,000 annual senior winter deaths ${ }^{1,2}$ and an economic burden of roughly 56 billion US dollars every year. ${ }^{3}$ Up to present, vaccination is the most effective method to prevent influenza infection. ${ }^{4}$ In our double blind randomized controlled trial (RCT), vaccination was shown effective in preventing influenza in the elderly.5, 6 However, there is a lack of direct evidence on its effectiveness on seasonal and long-term mortality.

Worldwide, only three RCTs have evaluated the efficacy of inactivated influenza vaccines on clinical influenza in community-dwelling elderly.5,8,9 None of these trials had the primary objective or power to assess mortality. Hence, current knowledge on mortality is based on observational research. Multiple recent meta-analyses of these studies concluded that vaccination can reduce mortality by 30 to 50 percent. ${ }^{10-12}$ However, observational studies are susceptible for selection bias and confounding. ${ }^{13-15}$ Therefore, various attempts have been made to reduce bias, for instance by adjusting for confounders, ${ }^{16,17}$ adjusting analyses, ${ }^{18,} 19$ and using advanced study designs. ${ }^{20}$ Despite this, critics state that the role of vaccination in the elderly is unclear and an adequately powered, placebo-controlled trial should be undertaken. ${ }^{20,21}$ However, such a trial is unlikely to gain ethical approval. $7,13,23$ Thus, conclusions on the effectiveness of influenza vaccination on mortality in community-dwelling elderly may remain based on observational studies and the inference that reduction of influenza implies reduction of influenza-related complications including mortality.

Besides the effects on short-term (i.e. seasonal or annual) mortality, the long-term potential of vaccination has never been evaluated in experimental settings. Studies have suggested that vaccination might prevent influenza-related complications such as cardiovascular events, ${ }^{24,25}$ reduce severity of community-acquired pneumonias, ${ }^{26}$ and reduce the risk of hospitalization. ${ }^{27,28}$ It has also been suggested that influenza vaccination might provide residual protection against influenza strains that occur later in life, ${ }^{29}$ and elicit immune memory. ${ }^{30,31}$ Hence, we hypothesize that by preventing (accumulation of) influenza-related complications and residual protection that lasts more than just one influenza season, influenza vaccination could reduce mortality on the longer term. Considering the age-associated decline of the immune system and the large group of influenza vaccination-naive patients by the time of conducting the trial, describing longterm outcome of influenza vaccination might be particularly interesting in the younger elderly.

Evaluating the long-term outcome of vaccination is relevant since it contributes to the evidence of the effects on mortality from a long-term perspective and might support patient and policy decision making. Therefore, we have used our 1991 trial as baseline for a long-term follow-up study to explore the long-term outcome of influenza vaccination on mortality and underlying causes of death in community-dwelling elderly. 


\section{METHODS}

We conducted a 25-year follow-up on mortality of an RCT on the efficacy of influenza vaccination in the elderly in the 1991/1992 winter season.

\section{Study population}

The 1991 trial involved 34 family physicians in 15 practices in the Netherlands. In total, 1,838 patients aged $>60$ years, not known to belong to those high-risk groups in which vaccination was recommended, were included. At that time, age was no criterion for recommending vaccination. Cardiovascular, pulmonary or metabolic problems that did not require vaccination according to the family physician were reported in 490 participants. Following randomization, patients received an intramuscular injection in the deltoid area with the inactivated quadrivalent influenza vaccine $(n=927)$ or with saline solution $(n=911)$. We refer to our original publication for more detailed information on the trial intervention. ${ }^{5}$

\section{Data collection}

We completed the 1991 trial data with mortality statistics. In the Netherlands, Statistics Netherlands (Centraal Bureau voor de Statistiek, CBS), keeps person records including information on the vital status and death certificates. Person records are linked to a unique identity number. To maximize the yield of the CBS search, we carried out an extensive genealogical search retrieving all relevant information useful for tracking identity numbers

\section{(S1 Text, S1 Fig. and S2 Fig.). A summary of the search is given in Fig.1.}

If data retrieved by the genealogic search conflicted with patient characteristics as reported in the 1991 trial (e.g. differences in age or sex), the conflicting cases were checked for clerical errors in the database by looking up the informed consent forms of the 1991 trial. If no clerical errors had been made and the genealogic search yielded one official person record only, then the patient characteristics were updated using the new information found. We defined six mortality endpoints based on the diagnostic codes of the International Classification of Diseases, Ninth Revision (ICD-9) for deaths prior to 1996 and ICD-10 for deaths that occurred in 1996 or later 32,33: all-cause deaths (all ICD codes), underlying respiratory deaths (ICD-9 codes 460-519, ICD-10 codes J00- J99), underlying circulatory deaths (ICD-9 390-459, ICD-10 codes 100-199), underlying respiratory or circulatory deaths, and underlying pneumonia or influenza deaths (ICD-9 codes 480-487, ICD-10 codes J09-J18). To ensure that influenza and pulmonary antecedents possibly related to influenza were noted, we modelled the sixth category ('pulmonary related mortality'); taking into account any pulmonary condition registered as antecedent or primary cause of death. All-cause mortality, primary and antecedent death causes were available up to 1 January 2017, 1 January 2016 and 1 January 2013 respectively. For each endpoint, seasonal mortality was assessed. Seasonal mortality was defined as any death occurring 
between December up to June, assuming that influenza-related mortality is likely to occur during or shortly after influenza epidemics, which in the Netherlands can take up until the end of April. ${ }^{34}$

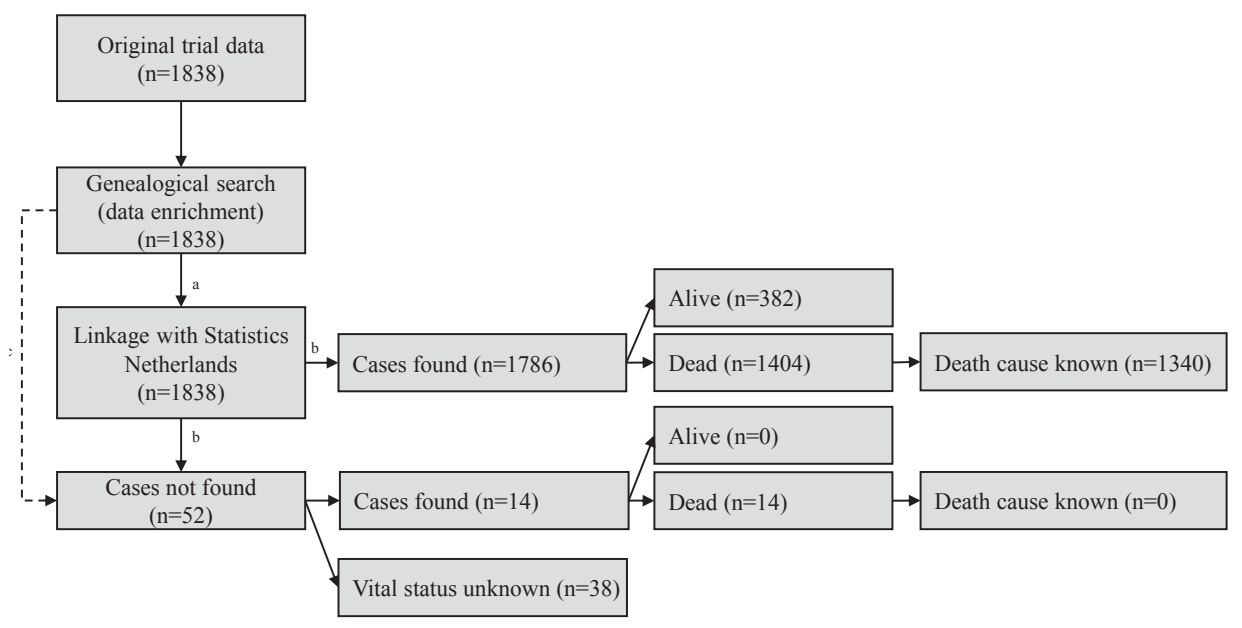

Fig.1. Process of data collection for obtaining mortality statistics.

a) If cases were not found by the CBS, data retrieved by the genealogical search was used in order to obtain follow-up information.

b) Genealogical search was used for maximizing CBS-search results.

c) Life status as known by 1 January, 2017.

Bias

Selection bias and confounding by indication at baseline were avoided since this study is based on double blind placebo-controlled randomization. ${ }^{5}$ If genealogical search data conflicted with personal records registered in the 1991 trial, a four member panel blinded for the trial intervention updated the personal records only after agreeing on data reliability. The randomization was maintained for all subjects, ensuring intention-to-treat analysis.

Variables

Eight baseline independent variables were considered, including: trial intervention (trial vaccination or placebo), age at trial intervention, sex, disease status as known in 1991, smoking status and previous vaccination status (influenza vaccination in 1989/1990 and 1990/1991). In the 1991 trial, participants with multiple comorbidities had been assigned to one single disease group only (e.g. a patient with coronary sclerosis and chronic obstructive pulmonary disease was assigned to disease group 'cardiovascular disease'). In order to optimize data usage in our followup study, we choose to reclassify the complete disease status as known in 1991 into each of the 
underlying disease clusters that had been registered, i.e.: cardiovascular disease, pulmonary disease and diabetes mellitus. Thus, a patient with coronary sclerosis and chronic obstructive pulmonary disease was now assigned to both 'cardiovascular disease' and 'pulmonary disease'.

\section{Statistical analysis}

Kaplan-Meier survival curves were plotted to illustrate the potential association between trial vaccination and survival. We performed univariate and multivariable Cox proportional hazards regression analyses to estimate the hazard ratio (HR) and 95\% confidence intervals for all-cause mortality by trial vaccination. Covariates were added as dichotomous variables (trial intervention, sex, cardiovascular disease, pulmonary disease, diabetes mellitus, previous vaccination status), ordinal (smoking status) or continuous variable (age at trial intervention). We used all covariates and all possible interaction terms in the multivariable Cox regression analyses, dropping the least significant terms by backward stepwise approach ( $p>0.05$ ). The proportional hazard assumption was checked comparing estimated $\ln (-\ln )$ survivor curves. To evaluate the relation between trial vaccination and seasonal or specific causes of death by means of sub-hazard ratios (SHRs), we used competing risk regression analysis, ${ }^{35}$ retaining those covariates and interaction terms with trial intervention (vaccine or placebo) in the model that appeared significant, unless stated otherwise. Since the numbers of events in competing risk regression analyses would be small, only the following additional interactions were assessed: age*sex, age*smoking status, age* cardiovascular disease, age* pulmonary disease, smoking status* pulmonary disease, smoking status*heart disease, smoking status*diabetes mellitus and pulmonary disease*heart disease. Statistical testing was based on two-sided testing with the use of a $5 \%$ significance level in all analyses. Subgroups based on age and medical conditions were analyzed for overall mortality only. Underlying causes of death were not analyzed as outcome since numbers of subgroups would be too small and multiple testing could induce false positive findings. Data were missing for smoking status ( $n=82$ ) and previous vaccination in 1990 ( $n=2)$. We analyzed our data by assigning the cases with missing smoking status to a separate group and excluding the latter two cases. In order to translate the potential survival benefit to clinical practice, we calculated the difference in median survival between groups. Since this approach was not recorded in our protocol beforehand, this will be considered of additive value. We used IBM SPSS version 23 for Cox regression analysis. Competing risk analyses were performed using competing risk package (cmprsk) in R statistical software, version 3.3.1 ( $R$ Foundation for Statistical Computing). If the CBS-search did not yield information on the vital status of a participant, we used alternative data sources (see result section) to retain a follow-up date and censored the case from that point onwards. To determine whether censoring before end of follow-up posed a potential threat to validity, we carried out sensitivity analyses applying two extreme scenarios; either all participants without actual vital status on 1 January, 2017 died after censoring, or lived up to 1 January, 2017. Our protocol was registered in the International Clinical Trials Registry Platform (ICTRP) under NTR6179, before data-analysis was performed. 


\section{Ethical considerations}

The 1991 trial participants had not been explicitly requested to approve for obtaining information on their vital status later on, since in 1991 this was beyond the scope of the trial. Concerning our follow-up study, the medical ethics committee of the Maastricht University Medical Center concluded this study was not subject to the Medical Research Involving Human Subjects Act. In addition, Statistics Netherlands approved anonymous processing of the mortality data.

As explained in more detail in our supplement (S1 Text), multiple institutional databases have been searched by institute officials: Statistics Netherlands, Municipal Personal Records Database (Gemeentelijke Basisadministratie, GBA), Netherlands Centre for Family History (Centraal Bureau voor Genealogie, CBG) and Regional Historical Centre of Limburg (Rijksarchief Limburg). The aim of the study was explained to each institutional board and/or official. The institutes decided whether mortality dates of the specific cases we requested for could legally be provided. Only after this decision, the databases were searched by the institutes' officials. No interviews of family members of the deceased participants have been performed in this study. In case former participants were not found in the official databases of the various institutions, the general practitioner was requested to provide this information on the life status (not the cause of death). The general practitioner and his/her staff were motivated to contribute to this study and went through patient records to retrieve information on the exact date of birth. In case of uncertainty, the general practitioners' staff called a family member of the deceased participant to inform about the date of death (not the death cause). The mortality data were accessed in a fully anonymized way. When mortality dates had been collected, the final step was obtaining the specific death cause of the participants by requesting Statistics Netherlands. Official permission to use these data was granted by the Committee of Statistics Netherlands (report available on request). Information on date of death was important for the CBS in order to search for personalized (and fully anonymized) identification numbers (unique codes corresponding to an unique person). These identification numbers where then used to obtain specific death causes, thus making it impossible to link a specific person to a specific death cause. Our analyses on death causes could only be performed on the online and secured server of Statistics Netherlands, thus ensuring patient privacy. Enriched data (containing information on death causes) could not be exported.

\section{RESULTS}

\section{Follow-up data}

The process of data collection is summarized in Fig.1. During 29,867 person-years of follow-up (median 17.34 years, 95\% Cl 16.67-18.01), 1,418/1,838 (77.1\%) subjects died. Information on cause of death was retrieved in 1,340/1,418 (94.5\%) cases. A total of 420 cases were censored of which 382 were alive at end of follow-up (January 1, 2017). Thus information on the known vital status was collected in 1,800/1,838 (97.9\%) cases. The remaining 38 cases with unknown 
vital status were divided equally between vaccine $(n=16)$ and placebo group $(n=22)$. Local municipalities provided a follow-up date (e.g. date of rehousing / migration) in 16 cases. In the 22 remaining cases, the last date recorded during the 1991 trial was used to set the censor date.

Characteristics of study population

Patient characteristics of the 1991 trial and the updated trial are shown in Table 1. Updating the trial data (see 'methods') did not result in any relevant differences between the intervention and placebo group.

Influence of influenza vaccination on mortality and specific causes of death

Median survival for vaccine group was 17.39 years (interquartile range 10.61-24.58) versus 17.20 years for placebo group (10.02-23.70); a difference of 2.2 months ( $95 \% \mathrm{Cl}-14.7$ to 19.1). Kaplan-Meier survival plots illustrating survival of different age groups are shown in Fig.2. Corresponding numbers at risk and hazard ratios are listed in Table 2. After adjusting for covariates and taking potential effect modifiers into account, this study found no overall effect of influenza vaccination on mortality (adjusted HR 0.95, 95\% CI 0.85-1.05). Sensitivity analyses (not presented) showed consistent results.

Overall hazard ratios contrasting trial vaccination versus placebo for different outcome measures are presented in Table 3. Neither overall nor seasonal differences between trial vaccination and placebo on all-cause mortality or any underlying cause of death were shown. The interaction term between trial vaccination and diabetes mellitus was significant $(p=0.02)$ for all-cause mortality. Also interaction terms between trial vaccination and pulmonary disease ( $p=0.04)$ and trial vaccination and smoking ( $p=0.04)$ were significant for pulmonary related seasonal mortality. Subsequent analysis for participants with and without pulmonary disease for this outcome demonstrated SHRs of 0.56 (95\% Cl 0.31-1.01) and 1.21 (95\% Cl 0.83-1.77) respectively (S3 Fig. and S4 Fig.). The interaction term between trial vaccination and smoking status was not retained in the model since there were no important differences in the SHRS for the model with and without interaction for each of the smoking categories. The SHR for all-cause mortality stratified by diabetes mellitus is shown in Table $\mathbf{4}$ and did not demonstrate a statistically significant effect of trial vaccination after adjusting the model to the small group size $(n=59)$. 
Table 1. Patient baseline characteristics of the trial population versus the updated trial population.

\begin{tabular}{|c|c|c|c|c|}
\hline \multirow[b]{2}{*}{ Variable } & \multicolumn{2}{|c|}{$\begin{array}{l}\text { Vaccine group ( } n=927) \\
\text { No.(\%) }\end{array}$} & \multicolumn{2}{|c|}{$\begin{array}{l}\text { Placebo group ( } n=911) \\
\text { No.(\%) }\end{array}$} \\
\hline & 1991 trial & $\begin{array}{l}\text { updated } \\
\text { trial }\end{array}$ & 1991 trial & $\begin{array}{l}\text { updated } \\
\text { trial }\end{array}$ \\
\hline \multicolumn{5}{|l|}{ Sex ${ }^{a}$} \\
\hline Male & $420(45.3)$ & $413(44.6)$ & $449(49.3)$ & $445(48.8)$ \\
\hline Female & $507(54.7)$ & $514(55.4)$ & $462(50.7)$ & $466(51.2)$ \\
\hline \multicolumn{5}{|l|}{ Age $^{a}$} \\
\hline $60-64$ & $368(39.7)$ & 379 (40.9) & $396(43.5)$ & $412(45.2)$ \\
\hline $65-69$ & $281(30.3)$ & $274(29.6)$ & 249 (27.3) & $240(26.3)$ \\
\hline $70+$ & $278(30.0)$ & $274(29.6)$ & $266(29.2)$ & $259(28.4)$ \\
\hline \multicolumn{5}{|c|}{ Smoking status (1991) } \\
\hline Never & $367(39.6)$ & $367(39.6)$ & $339(37.2)$ & $339(37.2)$ \\
\hline Stopped & $312(33.7)$ & $312(33.7)$ & $311(34.1)$ & $311(34.1)$ \\
\hline Actual & $206(22.2)$ & $206(22.2)$ & $221(24.3)$ & $221(24.3)$ \\
\hline Unknown & $42(4.5)$ & $42(4.5)$ & $40(4.4)$ & $40(4.4)$ \\
\hline \multicolumn{5}{|c|}{ Pulmonary disease (1991) b } \\
\hline Yes & $105(11.3)$ & $129(13.9)$ & $95(10.4)$ & $115(12.6)$ \\
\hline No & & $798(86.1)$ & & $796(87.4)$ \\
\hline \multicolumn{5}{|c|}{ Heart disease (1991) } \\
\hline Yes & $125(13.5)$ & $125(13.5)$ & $124(13.6)$ & $124(13.6)$ \\
\hline No & & $802(86.5)$ & & $787(86.4)$ \\
\hline \multicolumn{5}{|c|}{ Diabetic Mellitus (1991) } \\
\hline Yes & $21(2.3)$ & $27(2.9)$ & $20(2.2)$ & $32(3.5)$ \\
\hline Yes & & $900(97.1)$ & & $879(96.5)$ \\
\hline \multicolumn{5}{|c|}{ Previous vaccination (1989 and/or 1990)b } \\
\hline Yes & $118(12.7)$ & $119(12.9)$ & $120(13.2)$ & $119(13.1)$ \\
\hline No & 809 (87.3) & $807(87.1)$ & $791(86.8)$ & $791(86.9)$ \\
\hline Unknown & & $1(0.1)$ & & $1(0.1)$ \\
\hline
\end{tabular}

a) Differences in patient characteristics between 1991 trial and updated trial due to either clerical errors made in the original informed consent forms or incorrect data entry at time of trial registration.

b) Differences in patient characteristics between 1991 trial and updated trial due to reclassification of covariates. 
Table 2. Numbers at risk and hazard ratios corresponding to Kaplan-Meier plots for vaccinated and unvaccinated participants of the 1991 trial.

\begin{tabular}{lllll}
\hline Age group & Vaccine / placebo & Follow-up (years) & \\
& & $\mathbf{0}$ & $\mathbf{5}$ & $\mathbf{1 0}$ \\
\hline All ages & Vaccine & 927 & 835 & 708 \\
& Placebo & 911 & 812 & 677 \\
$60-64$ & Vaccine & 379 & 359 & 332 \\
& Placebo & 412 & 376 & 342 \\
$65-69$ & Vaccine & 274 & 252 & 227 \\
& Placebo & 240 & 214 & 185 \\
$70+$ & Vaccine & 274 & 224 & 149 \\
& Placebo & 259 & 222 & 150 \\
\hline
\end{tabular}

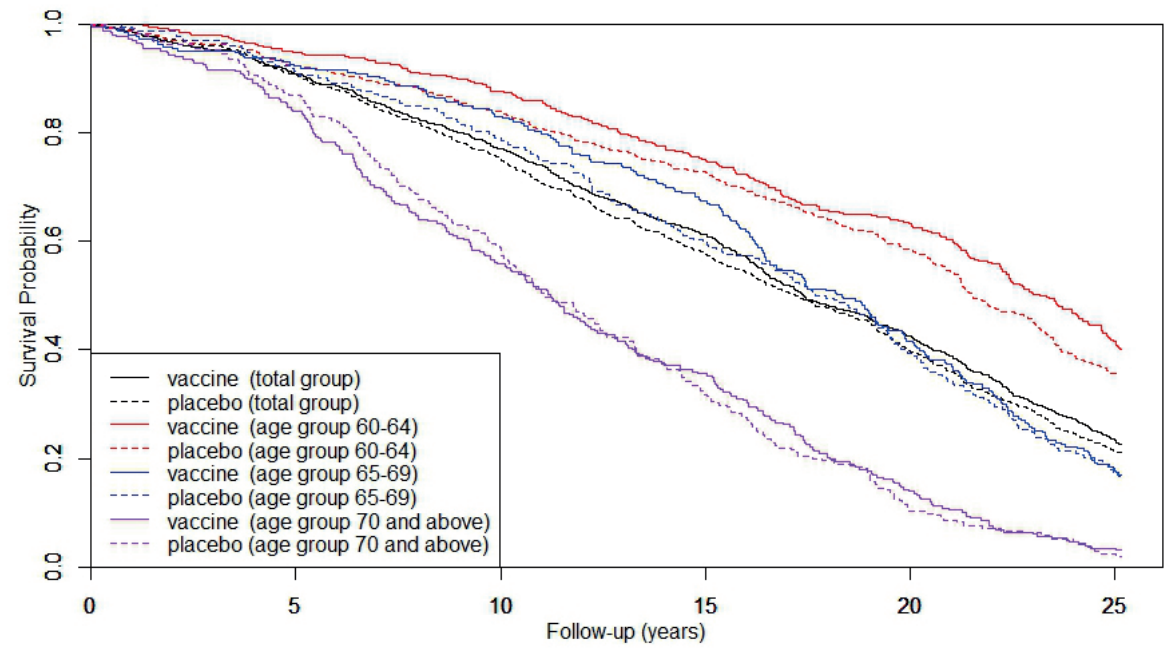

Fig.2. Kaplan-Meier plots for vaccine versus placebo group for different age groups (numbers at risk presented in Table 2). 


\begin{tabular}{lllll}
$\mathbf{1 5}$ & $\mathbf{2 0}$ & $\mathbf{2 5}$ & $\begin{array}{l}\text { Hazard Ratio } \\
(\mathbf{9 5 \%} \mathrm{Cl})\end{array}$ & P value \\
\hline 563 & 389 & 212 & $0.94(0.85-1.05)$ & 0.29 \\
518 & 357 & 187 & & \\
284 & 239 & 155 & $0.86(0.72-1.03)$ & 0.10 \\
297 & 239 & 142 & & \\
184 & 113 & 48 & $0.95(0.79-1.15)$ & 0.61 \\
140 & 92 & 40 & & \\
95 & 37 & 9 & $0.97(0.81-1.15)$ & 0.72 \\
81 & 26 & 5 & & \\
\hline
\end{tabular}

Subgroup analyses for different health conditions, previous vaccinations and age groups (Table 4), did not show a significant effect of vaccination on survival. Vaccination of the younger elderly (60-64 years) demonstrated the highest reduction in mortality rate; i.e. 14\% (adjusted HR 0.86, 95\% Cl 0.72-1.03). In this age group, median survival time was 23.18 years (3rd quartile 15.00) in the vaccine group versus 21.50 years in placebo group (3rd quartile 13.67), an absolute difference of 20.1 months (95\% Cl 2.4-37.9). Only the 3rd quartile of this group could be provided since at end of follow-up, over $25 \%$ of the population was still alive. 
Table 3. Adjusted and unadjusted hazard ratio (HR) and sub-hazard ratio (SHR) of trial vaccination for all-cause mortality and underlying cause of death respectively.

\begin{tabular}{|c|c|c|}
\hline \multirow[t]{2}{*}{ Outcome } & \multicolumn{2}{|c|}{ Vaccine group $(n=927)$} \\
\hline & No.(\%) & rate $^{b}$ \\
\hline \multicolumn{3}{|c|}{ All-cause mortality } \\
\hline Full year & $711(76.7)$ & 4.65 \\
\hline Seasonal ${ }^{a}$ & $378(40.8)$ & 2.47 \\
\hline \multicolumn{3}{|c|}{ Pulmonary or circulatory deaths } \\
\hline Full year & $329(35.5)$ & 2.18 \\
\hline Seasonal ${ }^{a}$ & 185(20.0) & 1.23 \\
\hline \multicolumn{3}{|c|}{ Pulmonary deaths } \\
\hline Full year & $80(8.6)$ & 0.53 \\
\hline Seasonala & $56(6.0)$ & 0.37 \\
\hline \multicolumn{3}{|c|}{ Circulatory deaths } \\
\hline Full year & $249(26.9)$ & 1.65 \\
\hline Seasonala & 129(13.9) & 0.86 \\
\hline \multicolumn{3}{|c|}{ Influenza/pneumonia deaths } \\
\hline Full year & $35(3.8)$ & 0.23 \\
\hline Seasonala & $25(2.7)$ & 0.17 \\
\hline \multicolumn{3}{|c|}{ Pulmonary related deaths } \\
\hline Full year & $140(15.1)$ & 0.99 \\
\hline Seasonal ${ }^{a}$ & $81(8.7)$ & 0.57 \\
\hline
\end{tabular}

HR: hazard ratio; SHR: sub-hazard ratio.

Percentages are calculated by: $\mathrm{n}(\text { events) } / \mathrm{N} \text { (patients). } \mathrm{N} \text { (patients) })_{\text {vaccine }}=927, \mathrm{~N}$ (patients) $)_{\text {placebo }}=911$.

a) (Sub-) hazard ratios adjusted for age, sex, smoking status, pulmonary disease, heart disease, diabetes mellitus, previous vaccination status and first-order interactions: trial vaccination by all covariates and 8 additional interactions (i.e. age*sex, age*smoking status, age* pulmonary disease, age*heart disease, smoking status*pulmonary disease, smoking status*heart disease, smoking status*diabetes mellitus, pulmonary disease*heart disease), unless otherwise specified. Two individuals were excluded due to missing data. 


\section{Placebo group $(n=911)$}

No.(\%)

rate $^{b}$

Crude HR (95\% Cl)

Adjusted HR (95\% CI)

707(77.6)

4.85

0.94 (0.85-1.05)

$0.95(0.85-1.05)^{\mathrm{c}, \mathrm{d}}$

$381(41.8)$

2.61

$0.95(0.83-1.10)$

0.94 (0.81-1.08)

324(35.6)

2.25

0.99 (0.85-1.15)

$0.96(0.82-1.13)$

188(20.6)

1.31

$0.95(0.78-1.17)$

0.93(0.75-1.14)

75(8.2)

0.52

$1.04(0.76-1.43)$

$1.04(0.76-1.44)$

50(5.5)

0.35

$1.10(0.75-1.60)$

$1.06(0.72-1.56)$

249(27.3)

1.73

$0.97(0.81-1.16)$

$0.96(0.80-1.15)$

138(15.1)

0.96

$0.90(0.71-1.15)$

$0.90(0.71-1.15)$

28(3.1)

0.19

$1.22(0.74-2.01)$

$1.20(0.73-1.97)$

21(2.3)

0.15

$1.16(0.65-2.07)$

$1.13(0.63-2.03)^{\mathrm{e}}$

134(14.7)

0.99

$1.02(0.81-1.29)$

1.07(0.84-1.37)

86(9.4)

0.63

$0.92(0.68-1.24)$

$0.96(0.70-1.31)^{f}$

b) Defined as death occurring annually between the $1^{\text {st }}$ of December and the $31^{\text {st }}$ of May.

c) (Sub-) hazard ratio adjusted for all potential first-order interactions, given the large numbers of events $(n=1418)$.

d) Significant interaction term $(p=0.02)$ between trial vaccination and diabetes mellitus.

e) No adjustments made for interactions, given the small numbers of events $(n=46)$.

f) Significant interaction term $(p=0.04)$ between trial vaccination and pulmonary disease, and between trial vaccination and smoking $(p=0.04)$. 
Table 4. Adjusted and unadjusted hazard ratio (HR) for all-cause mortality by subgroups by trial vaccination.

\begin{tabular}{ll}
\hline & No.events / total (\%) \\
\cline { 2 - 2 } Subgroups & Vaccine group \\
\hline Age 60-64 (n=791) & $226 / 379(59.6)$ \\
Age 65-69 (n=514) & $226 / 274(82.5)$ \\
Age 70+ (n=533) & $259 / 274(94.5)$ \\
Pulmonary disease ( $n=244)$ & $112 / 129(86.8)$ \\
Cardiovascular disease ( $=249)$ & $107 / 125(85.6)$ \\
Diabetes ( $n=59)$ & $26 / 27(96.3)$ \\
Pulmonary and/or cardiovasc. disease and/or DM (n=490) & $218 / 251(86.9)$ \\
Previous vaccination ( $n=238)$ & $101 / 119(84.9)$ \\
\hline
\end{tabular}

HR: hazard ratio; DM: diabetes mellitus.

Data are $\mathrm{n}($ events)/N(patients).

\section{DISCUSSION}

In this study based on an RCT, we could not demonstrate a relation between one single influenza vaccination and mortality or specific causes of death in the elderly after 25 years of followup. Also no effect of vaccination on seasonal mortality was shown. Although not statistically significant, survival was in favor of the vaccine group during the entire length of follow-up, with hazard rates being constant over time.

Subgroup analyses suggested that the effects of vaccination on mortality could be more prominent in those aged 60-64 years (adjusted HR 0.86,95\% CI 0.72-1.03). This is supported by literature stating that influenza-specific humoral immunity and clinical effectiveness of influenza vaccination are negatively influenced by age. ${ }^{36-38}$ Analyzing the younger elderly as such is relevant given the ongoing debate on the influence of age on vaccination effectiveness. Moreover, influenza-associated mortality and hospitalization are substantial also in this group. ${ }^{39}$ Our finding of a difference in median survival, i.e. 20.1 months (95\% Cl 2.4-37.9), could be considered additional supportive evidence, not as proof of efficacy in this group.

Other subgroup analyses based on pre-existing cardiovascular and/or pulmonary disease or on previous vaccination did not show clear indications for effects of vaccination on all-cause mortality. Although patients with lung disease might be more prone to harmful effects of influenza, we could not demonstrate a clear effect of vaccination on pulmonary-related seasonal mortality in this subgroup (adjusted SHR 0.56, 95\% C 0.31-1.01). We found a sub-hazard ratio for vaccination of 1.53 (95\% $\mathrm{Cl} 0.89-2.62)$ in diabetic patients. However, this analysis is based on low numbers ( $n=59$ ) and the survival plots for diabetics showed an unfavorable effect of vaccination just 15 years after trial intervention (S5 Fig.). 
Crude HR (95\% CI)

\section{Placebo group}

262/412(63.6)

195/240(81.3)

250/259(96.5)

101/115(87.8)

$110 / 124(88.7)$

28/32(87.5)

209/239(87.4)

95/119(79.8)

\section{Adjusted HR $(95 \% \mathrm{Cl})^{\mathrm{a}}$}

$0.86(0.72-1.03)$

$0.99(0.82-1.20)$

1.04(0.87-1.23)

1.13(0.86-1.48)

$0.88(0.67-1.15)$

1.53(0.89-2.62)

0.97(0.80-1.17)

$1.01(0.76-1.34)$

a) Hazard ratios adjusted for sex, age, smoking status, pulmonary disease, heart disease, diabetes mellitus and previous vaccinations (two individuals were excluded due to missing data).

Despite the biological plausibility that vaccinating the youngest elderly would be more effective, it may seem unlikely that the effect size of trial vaccination on mortality in the younger elderly ( $\mathrm{HR} 0.86,95 \%$ Cl 0.72-1.03) is explained by administration of one single vaccination in 1991/1992. However, by that time uptake of influenza vaccinations was low and vaccination recommendations for those aged $>65$ were only introduced in 1996. Thus, especially for those aged 60-64, the 1991 trial vaccination was likely to have been the first and for a long time only influenza vaccination received. Since similar vaccine components as used in the 1991 trial showed to provide protection against some of the influenza strains that circulated the five subsequent years after trial intervention, this might have had implications for the longterm efficacy of the trial vaccination. For instance, it has been shown that influenza vaccines can elicit immune memory and protect against drift variants or non-homologous strains. ${ }^{30,31}$ Also, McLean et al. demonstrated that protection against influenza could also be elicited by vaccination during the previous season. This residual protection even occurred despite that the predominant viruses were antigenically distinct from previous season vaccine components. ${ }^{29}$ It should be stated that these studies included mainly young(er) individuals and thus might not fully apply to elderly due to their diminished immune responses. ${ }^{40}$ Yet eventually, we cannot ignore that in our trial, random allocation to vaccine or placebo group is at the basis of differences in survival between vaccine and placebo group in the younger aged. In addition, subgroup analyses of the age groups 65-69 and 70+ showed hazard ratios near 1.0. Taking these differences and the study power into account, in our view a protective effect of vaccination on mortality in the youngest age group cannot be ruled out.

Methodologically, it is difficult to compare our study results with literature since studies predominantly evaluate seasonal effects of vaccination on the short term. In their RCT, Praditsuwan et al. evaluated mortality after one year. Whereas underpowered for this secondary 
endpoint, they could not demonstrate a relation between vaccination and mortality. A cohort study showed that vaccination was associated with lower mortality in community-dwelling elderly during six months of follow-up, but did not prevent death in the subgroup of healthy elderly (relative risk 0.87, 95\% Cl 0.62-1.20). ${ }^{41}$ However, in that study vaccination did reduce mortality in elderly with comorbidity (relative risk 0.67, 95\% Cl 0.48-0.94). Moreover, a metaanalysis evaluating the effect of (different types of) influenza vaccination in a diverse population (various ages, largely elderly known with cardiovascular comorbidity), showed that influenza vaccination may reduce combined cardiovascular mortality. ${ }^{24}$ Our study did not demonstrate such a comorbidity-related subgroup effect, but may be difficult to compare with these studies since medical problems registered in our 1991 trial did not require vaccination according to the physician and thus might have been of minor clinical relevance.

A limitation of our study concerns its power. The 1991 trial was not designed to evaluate short-term mortality and thus numbers are too small to relate vaccination directly to influenza deaths in the influenza season that year. The high rate of complete follow-up (97.9\%) did result in high numbers of person years of follow-up and events, thus increasing the study power. However, post-hoc power calculation showed that only hazard ratios $>0.85$ could have been proven significant. Therefore, we cannot exclude that our group size was too small to be able to show effects of vaccination on mortality that are relevant at the population level.

It would have been interesting if more information on revaccination after 1991 was available because this may have affected mortality. Since in the Netherlands the legal obligation to store medical files expires after 15 years, reconstruction of these data is problematic. Because participants were not de-blinded at end of the trial, both randomized groups are likely to have been (re)vaccinated in equal proportions. Since differences in vaccination status between vaccination and placebo group will have become smaller over time, it is likely this has diluted the effect of our 1991 trial vaccination.

An important strength of our study concerns the follow-up being based on a welldocumented RCT. In this trial the vaccine was well matched with the epidemic influenza strains, possibly maximizing the efficacy of vaccination on morbidity, and - as set out in our hypothesis - on long-term mortality as an outcome. Since our trial was found to be the only one of the three previously mentioned RCTs that could be evaluated on long-term mortality and new placebocontrolled trials are unlikely to be approved by ethical boards, the results from our study might remain the only direct evidence available on long-term effects of influenza vaccination on mortality in community-dwelling elderly. 


\section{CONCLUSION}

In conclusion, this study did not demonstrate an effect of influenza vaccination on long-term mortality in community-dwelling elderly in general. However, taking the limited power of our study into account, the consistent and relevant findings in the subgroup aged 60-64 years merit a long-term study of the effect of vaccination on mortality the elderly. Therefore, we recommend researchers intending to conduct studies on the efficacy of influenza vaccines, to accustom these studies for longer follow-up. Moreover, these results could encourage researchers to compare long-term survival in younger and older vaccinated elderly and to study the long-term effect of influenza vaccination on immune memory. 


\section{REFERENCES}

1. Simonsen L, Reichert TA, Viboud C, Blackwelder WC, Taylor RJ, Miller MA. Impact of influenza vaccination on seasonal mortality in the US elderly population. Arch Intern Med 2005;165:265-72.

2. Thompson WW, Shay DK, Weintraub E, et al. Mortality associated with influenza and respiratory syncytial virus in the United States. JAMA 2003;289:179-86.

3. Molinari NA, Ortega-Sanchez IR, Messonnier ML, et al. The annual impact of seasonal influenza in the US: measuring disease burden and costs. Vaccine 2007;25:5086-96.

4. Paules C, Subbarao K. Influenza. Lancet 2017;390:697-708.

5. Govaert TM, Thijs CT, Masurel N, et al. The efficacy of influenza vaccination in elderly individuals. A randomized double-blind placebo-controlled trial. JAMA 1994;272:1661-5.

6. Govaert TM, Dinant GJ, Aretz K, et al. Adverse reactions to influenza vaccine in elderly people: randomised double blind placebo controlled trial. BMJ 1993;307:988-90.

7. Simonsen L, Taylor RJ, Viboud C, Miller MA, Jackson LA. Mortality benefits of influenza vaccination in elderly people: an ongoing controversy. Lancet Infect Dis 2007;7:658-66.

8. Allsup S, Haycox A, Regan M, Gosney M. Is influenza vaccination cost effective for healthy people between ages 65 and 74 years? A randomised controlled trial. Vaccine 2004;23:639-45.

9. Praditsuwan R, Assantachai P, Wasi C, Puthavatana P, Kositanont U. The efficacy and effectiveness of influenza vaccination among Thai elderly persons living in the community. J Med Assoc Thai 2005;88:256-64.

10. Chan TC, Fan-Ngai Hung I, Ka-Hay Luk J, Chu LW, Hon-Wai Chan F. Effectiveness of influenza vaccination in institutionalized older adults: a systematic review. J Am Med Dir Assoc 2014;15:226. e1-6.

11. Beyer WE, McElhaney J, Smith DJ, et al. Cochrane re-arranged: support for policies to vaccinate elderly people against influenza. Vaccine 2013;31:6030-3.

12. Darvishian M, Gefenaite $G$, Turner RM, et al. After adjusting for bias in meta-analysis seasonal influenza vaccine remains effective in community-dwelling elderly. J Clin Epidemiol 2014;67:734-44.

13. Baxter R1, Lee J, Fireman B. Evidence of bias in studies of influenza vaccine effectiveness in elderly patients. J Infect Dis. 2010;201:186-9.

14. Jackson LA, Jackson ML, Nelson JC, Neuzil KM, Weiss NS. Evidence of bias in estimates of influenza vaccine effectiveness in seniors. Int J Epidemiol 2006;35:337-44.

15. Simonsen L, Viboud C, Taylor RJ, Miller MA, Jackson L. Influenza vaccination and mortality benefits: new insights, new opportunities. Vaccine 2009;27:6300-4.

16. Groenwold RH, Hoes AW, Nichol KL, Hak E. Quantifying the potential role of unmeasured confounders: the example of influenza vaccination. Int J Epidemiol 2008;37:1422-9.

17. Armstrong BG, Mangtani P, Fletcher A, et al. Effect of influenza vaccination on excess deaths occurring during periods of high circulation of influenza: cohort study in elderly people. BMJ 2004;329:660.

18. Jansen AG, Sanders EA, Nichol KL, et al. Decline in influenza-associated mortality among Dutch elderly following the introduction of a nationwide vaccination program. Vaccine 2008;26:5567-74.

19. Fireman B, Lee J, Lewis $N$, et al. Influenza vaccination and mortality: differentiating vaccine effects from bias. Am J Epidemiol 2009;170:650-6.

20. Darvishian $M$, van den Heuvel ER, Bissielo A, et al. Effectiveness of seasonal influenza vaccination in community-dwelling elderly people: an individual participant data meta-analysis of test-negative design case-control studies. Lancet Respir Med 2017;5:200-11. 
21. Jefferson T, Di Pietrantonj C, Al-Ansary LA, et al. Vaccines for preventing influenza in the elderly. Cochrane Database Syst Rev 2010:CD004876.

22. Demicheli $V$, Jefferson T, Di Pietrantonj $C$, et al. Vaccines for preventing influenza in the elderly. Cochrane Database Syst Rev 2018;2:Cd004876.

23. Knottnerus JA. Influenza vaccination in the elderly: current evidence and uncertainties. J Clin Epidemiol 2009;62:675-6.

24. Clar C, Oseni Z, Flowers N, Keshtkar-Jahromi M, Rees K. Influenza vaccines for preventing cardiovascular disease. Cochrane Database Syst Rev 2015;5:CD005050.

25. Udell JA, Zawi R, Bhatt DL, et al. Association between influenza vaccination and cardiovascular outcomes in high-risk patients: a meta-analysis. JAMA 2013;310:1711-20.

26. Tessmer A, Welte T, Schmidt-Ott R, et al. Influenza vaccination is associated with reduced severity of community-acquired pneumonia. Eur Respir J 2011;38:147-53.

27. Nordin J, Mullooly J, Poblete S, et al. Influenza vaccine effectiveness in preventing hospitalizations and deaths in persons 65 years or older in Minnesota, New York, and Oregon: data from 3 health plans. J Infect Dis. 2001;184:665-70.

28. Nichol KL, Nordin J, Mullooly J, et al. Influenza vaccination and reduction in hospitalizations for cardiac disease and stroke among the elderly. N Engl J Med 2003;348:1322-32.

29. McLean HQ, Thompson MG, Sundaram ME, et al. Impact of repeated vaccination on vaccine effectiveness against influenza A(H3N2) and B during 8 seasons. Clin Infect Dis 2014;59:1375-85.

30. Dou Y, Fu B, Sun R, et al. Influenza vaccine induces intracellular immune memory of human NK cells. PLoS One 2015;10:e0121258.

31. Luytjes W, Enouf V, Schipper M, et al. HI responses induced by seasonal influenza vaccination are associated with clinical protection and with seroprotection against non-homologous strains. Vaccine 2012;30:5262-9.

32. World Health Organization. International Classification of Diseases, Ninth Revision. Geneva, Switzerland: World Health Organization.1977.

33. World Health Organization. International Statistical Classification of Diseases and Related Health Problems, Tenth Revision. Geneva, Switzerland: World Health Organization.1992.

34. Claas EC, de Jong JC, Bartelds AI, et al. [Influenza in the 1994/95 season; composition of vaccine for the 1995/96 season]. Ned Tijdschr Geneeskd 1995;139:2154-8.

35. Fine JP, Gray RJ. A Proportional Hazards Model for the Subdistribution of a Competing Risk. Am Stat Assoc 1999;94:496-509.

36. Gavazzi G, Krause KH. Ageing and infection. Lancet Infect Dis 2002;2:659-66.

37. Haralambieva IH, Painter SD, Kennedy RB, et al. The impact of immunosenescence on humoral immune response variation after influenzaA/H1N1 vaccination in older subjects. PLoS One 2015;10:e0122282.

38. Thomas RE. Are influenza-associated morbidity and mortality estimates for those $>/=65$ in statistical databases accurate, and an appropriate test of influenza vaccine effectiveness? Vaccine 2014;32:6884901.

39. Jansen AG, Sanders EA, Hoes AW, van Loon AM, Hak E. Influenza- and respiratory syncytial virusassociated mortality and hospitalisations. Eur Respir J 2007;30:1158-66.

40. Goodwin K, Viboud C, Simonsen L. Antibody response to influenza vaccination in the elderly: a quantitative review. Vaccine 2006;24:1159-69.

41. Voordouw BC, van der Linden PD, Simonian S, et al. Influenza vaccination in community-dwelling elderly: impact on mortality and influenza-associated morbidity. Arch Intern Med 2003;163:1089-94. 


\section{SUPPLEMENTARY INFORMATION}

\section{S1 Text. Method used for genealogical data collection.}

An extensive genealogical search was conducted in order to maximize the yield of the search performed by Statistics Netherlands (CBS). This genealogical search is summarized in supplement S1 Fig. Since multiple institutes were involved in the process of data collection, an explanatory box is shown below this text. On our request the Municipal Personal Records Database (Gemeentelijke Basisadministratie, GBA) was searched by a government official in December 2015 to identify whether the participants had died, and provide the date of death if applicable (i.e. the 'vital status'). In case of negative findings we contacted the participants' family practice to enquire about the vital status. If this could not be provided (e.g. due to migration), we requested the Netherlands Centre for Family History (Centraal Bureau voor Genealogie, CBG) and subsequently, an archivist of the Regional Historical Centre of Limburg (Rijksarchief Limburg) to conduct a search in the national and the regional archives. If a search for an individual was not successful, the vital status was checked by the local municipality where the former participant had been living according to our most recent information. Search results of the family practice were double checked by the municipalities. In case patient characteristics retrieved by our genealogical search conflicted with the characteristics as recorded in our database originating from 1991, we checked the original informed consent forms for inconsistencies and adjusted the data based on these findings. Finally, Statistics Netherlands obtained the unique identification numbers based on our genealogic search (S2 Fig.). These numbers were then used to look up the date of death and underlying death cause in the CBS-database.

\section{Explanatory box. Institutes that collect and preserve mortality data in the Netherlands.}

- The Municipal Personal Records Database (Gemeentelijke Basisadministratie, GBA) The national database maintained under the authority of the Dutch Ministry of the Interior and Kingdom Relations, that digitally registers official personal records of all Dutch residents and residents who passed away after October 1994.

- $\quad$ Statistics Netherlands (Centraal Bureau voor de Statistiek, CBS) The national autonomous administrative body that is responsible for collecting and processing national data in order to publish official statistics.

- Regional Historical Centre of Limburg (Rijksarchief Limburg) A statutory authority that collects and preserves a wide range of documents relating to the history of Limburg and its people for the purpose of the public and regional municipalities.

- Netherlands Centre for Family History (Centraal Bureau voor Genealogie, CBG) Both an information center and center of expertise, the CBG provides sources, advice and research in the field of genealogy, heraldry, family names and related domains in the Netherlands. 


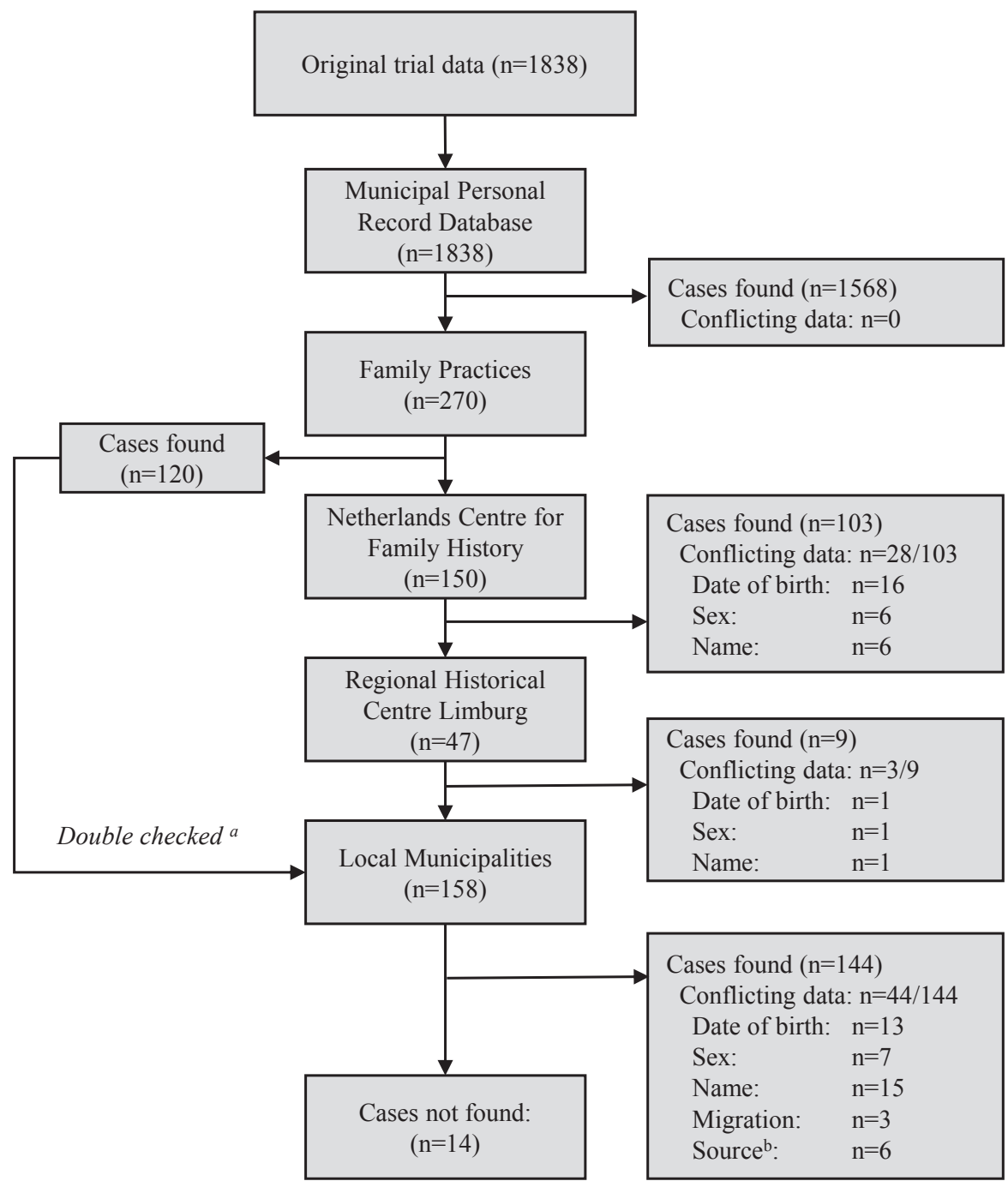

\section{S1 Fig. Flow chart indicating process of data enrichment by genealogical search.}

Results from the genealogical search provided input for the definite search as performed by Statistics Netherlands (S2 Fig.).

a) Registration of personal records by family practices can be considered indicative but not conformational for verity of personal data. Therefore, details provided by family practices were double checked by local municipalities.

b) Conflicting source indicates that the used data source shows differences in personal data (e.g. different date of birth or sex) when compared to original trial data (as registered in 1991). 


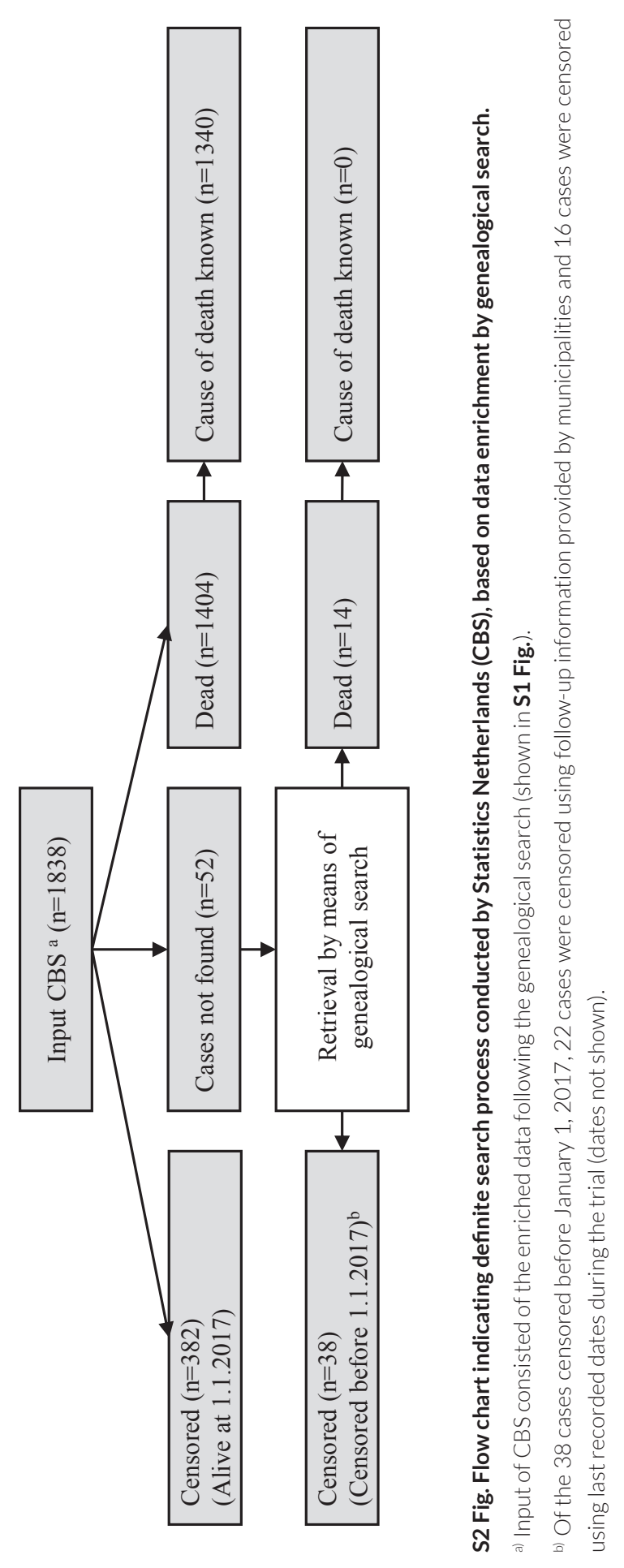




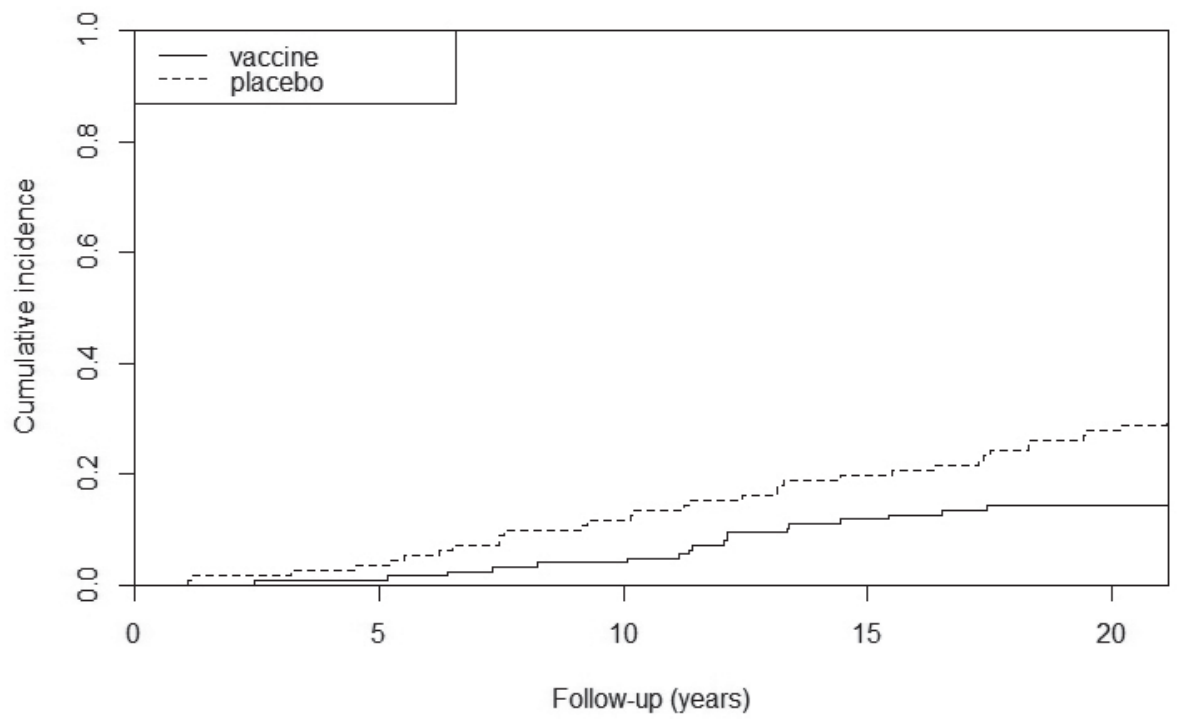

S3 Fig. Cumulative incidence plot indicating the incidence of pulmonary related seasonal deaths in participants with lung disease $(n=244)$.

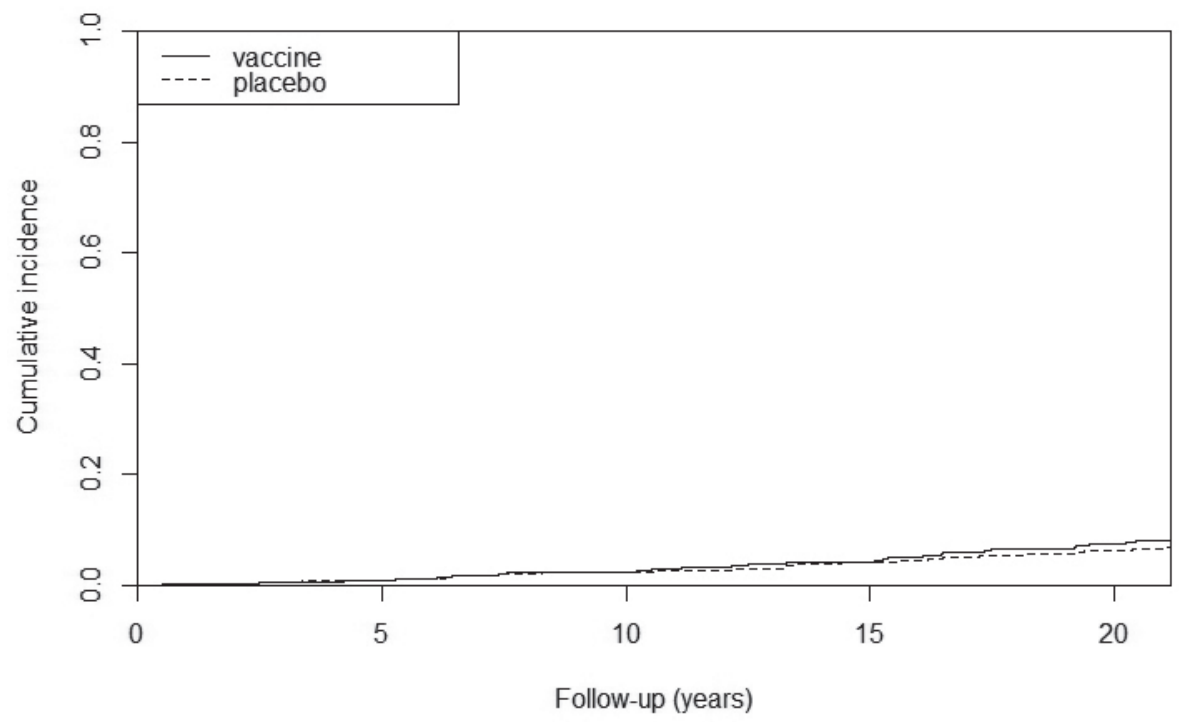

S4 Fig. Cumulative incidence plot indicating the incidence of pulmonary related seasonal deaths in participants without lung disease $(n=1,594)$. 


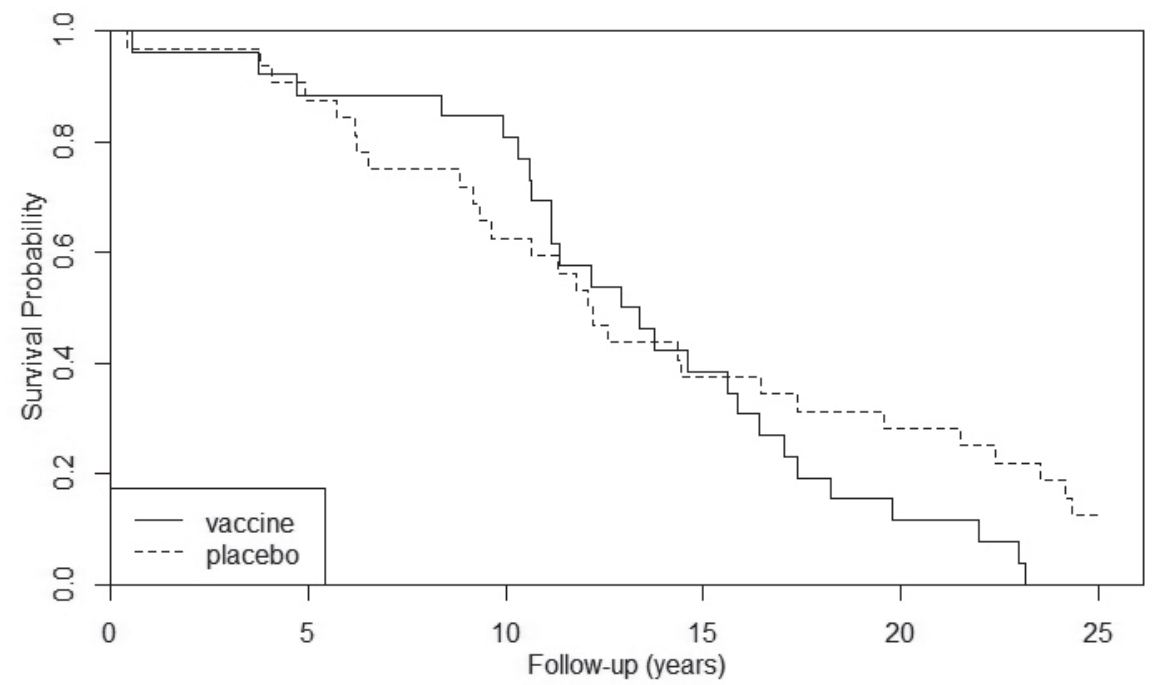

S5 Fig. Crude Kaplan-Meier survival curves for vaccinated and unvaccinated diabetic patients $(n=59)$.

An inverse relation between vaccination and survival after approximately 15 years of follow-up is shown. 



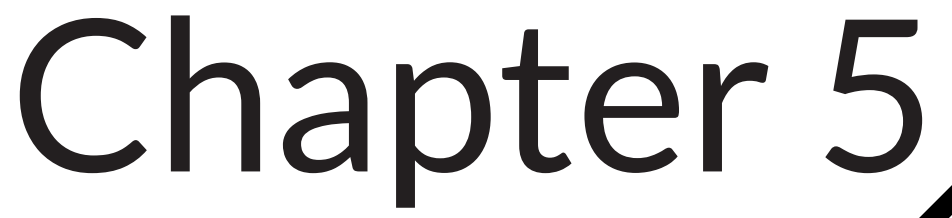

Antibody response follow ng influenza vaccination nthe elderly and long-tarm sur ival: a follow-up stud based on a randomistrolled trial

Ruud A.F. Verhees

Carel Thijs

Walter Beyer

Johannes A. Knottnerus

Geert-Jan Dinant

Submitted 



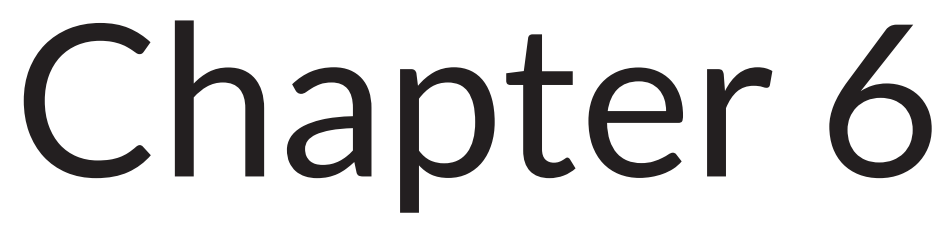

\title{
Influenza point-of-care tests
} in the general practice and emergency department: analytical accuracy and added value*

\author{
A narrative review \\ Ruud A.F. Verhees \\ Suzanne P.M. Lutgens \\ Ron Kusters \\ Geert-Jan Dinant \\ Jochen W.L. Cals
}

* Chapter translated from:

Ned Tijdschr Geneeskd. 2019;163:D3806 


\section{ABSTRACT}

There are various point-of-care (POC) tests available that allow the diagnosis of influenza within circa 30 minutes. POC tests that utilise antigen detection are user-friendly, but do have analytical accuracy limitations. POC tests based on polymerase chain reaction (PCR) technique are accurate, but rely on direct support from a medical laboratory; therefore, these tests can only be deployed in hospitals. There is limited direct evidence for a clinical effectiveness of the influenza POC tests in the emergency department (ED) and there is a lack of proof concerning the clinical effectiveness of the influenza POC test in a general practitioners (GP) practice setting. Influenza POC tests can reduce patients' length-of-stay in the ED and improve patient flow, provided there is capacity to accommodate this. Based on current available literature, it is warranted to deploy the PCR-based influenza POC test in an ED setting. However, presently the implementation of the influenza POC test in GP-practices cannot be justified. 


\section{INTRODUCTION}

Influenza causes significant morbidity and mortality, especially among the elderly and chronically ill. During flu seasons, the workload of physicians and nurses in primary and secondary care increases dramatically, as do occupancy of (isolation) beds and length of hospitalization. This can lead to capacity problems and obstruction of the care chain. It is well possible that the demand for care will increase further in the future as a result of the declining influenza vaccination rate among the elderly. To cope with the higher demand for care, it is important to critically review the referral and admission criteria for patients with influenza symptoms. After all, influenza symptoms usually do not appear until several days after exposure.

Can rapid tests - also known as point-of-care (POC) tests - for influenza be of added value to general practitioners (GPs) and emergency departments (EDs)? In this article, we describe the accuracy of rapid influenza diagnostic tests and their effect on clinically relevant outcome measures, such as use of antibiotics and antivirals, length of stay at the emergency department and length of hospitalization.

\section{Influenza POC test}

A POC test is a biomedical test performed on patient material (e.g. saliva, blood, urine or faeces). Such tests can guide clinical decision-making as they can be performed during or immediately before or after consultation, and normally the result is quickly available. As clinical decision-making takes place at different times depending on the setting, the term 'POC test' is used in both primary and secondary care. The analysis time of the tests may differ, however. Several POC tests have become available in recent years. The U.S. Food and Drug Administration has approved three rapid influenza diagnostic tests that use a polymerase chain reaction (PCR) technique and produce results within 30 min. However, the majority of influenza POC tests rely on antigen detection and use monoclonal antibodies against different viral nucleoproteins. These tests are generally smaller (the size of a matchbox), less expensive and technically easier to perform.

\section{Potential advantages}

Influenza-like symptoms can be caused by either viral or bacterial respiratory infections. Influenza is not easily diagnosed merely on the basis of symptoms, and the different pathogens of respiratory tract infections are difficult to distinguish clinically. Conventional laboratory tests often provide information too late to directly influence treatment decisions. Although the POC C-reactive protein (CRP) test can differentiate severe from self-limiting lower respiratory tract infections, it is a weak predictor of the type of pathogen. 
Long waits for test results or even failure to perform influenza diagnostics can not only lead to defensive diagnostics and antibiotic treatment, but also to unnecessary referral, admission and isolation of patients with influenza-like symptoms. Influenza POC tests may improve patient care (faster decision-making on the right treatment and right treatment location), reduce the risk of obstruction of the care chain during influenza epidemics, and bring down healthcare costs and the workload of healthcare providers.

\section{Current practice}

Blood culture, i.e. the culture of micro-organisms from blood for laboratory diagnosis, which was once the gold standard for the diagnosis of influenza, has been replaced by the reverse transcription PCR-test, partly because of its high sensitivity and shorter analysis time. As influenza viruses consist of single-stranded RNA, complementary DNA must first be produced by means of a 'reverse transcriptase' reaction, on which PCR can then be performed. Less frequent is the use of antigen detection tests. Serological tests are less suitable for diagnosing influenza, as two to three weeks must elapse before a titre rise can be demonstrated.

General practitioners diagnose influenza mainly on the basis of history taking and physical examination. Enquiries at a large laboratory with extensive services for primary care in the southeast of the province of North Brabant show that GPs hardly ever use specific influenza diagnostics: the more than 700 GPs practising in this area requested only four PCR-tests for influenza in the period from 1 January 2017 to the summer of 2018. The practice manual entitled 'Influenza Vaccination' published by the Dutch College of General Practitioners (NHG) and the National Influenza Prevention Programme Foundation does not contain any recommendations concerning influenza diagnostics. The guidelines of the U.S. Centers for Disease Control and Prevention (CDC), on the other hand, do recommend testing patients with influenza-like symptoms when they are referred to hospital.

During the flu season in the Netherlands, it is common practice to perform a PCR-test for influenza in patients with fever and influenza-like symptoms who are admitted to an emergency department, because they have to be cared for in isolation. In the winter of 2017-2018, influenza POC tests based on PCR performed at the emergency departments of several Dutch hospitals, in addition to regular PCR-tests. During the flu season, the number of patients who were admitted to hospital dropped. During the 2018-2019 flu season, the influenza POC tests based on PCR was used as a regular diagnostic tool in several hospitals.

\section{Analytical accuracy}

We already indicated that influenza is difficult to diagnose merely on the basis of clinical symptoms. In order to determine the analytical accuracy of a rapid influenza test, it is therefore essential to compare the result of the rapid test with that of the best available test (i.e. a PCRtest). 
The analytical accuracy of influenza POC test is influenced by several factors, such as viral load and influenza prevalence. The viral load is generally higher in children, patients with severe infection and at the onset of the disease. In the event of a high influenza incidence, the influenza POC test will have a higher positive predictive value and a lower negative predictive value. Calculation examples are provided in Supplement 1 to this article.

Due to variation in test settings, test types, target populations and virus types, influenza POC tests have a sensitivity ranging from 20-100\% and a specificity ranging from 54100\%. Influenza POC tests based on PCR are only used in hospitals and generally score better (sensitivity and specificity often >95\%) than the POC antigen detection tests.

In general practice, only the analytical accuracy of influenza POC tests using antigen detection has been investigated to date. The analytical accuracy of these tests is limited (sensitivity: 40-70\% (extremes: 10-80); specificity: 90-95\% (extremes: 85-100)); these findings match the CDC's opinion regarding the limited analytical accuracy.

\section{Randomized studies}

We searched PubMed on 2 March 2019 for randomized studies on the effect of influenza $\mathrm{POC}$ tests on at least one of the clinically relevant outcome measures (use of antibiotics and antivirals, length of stay at the emergency department and length of hospitalization). We used the following syntax: ((( (influenza OR "influenza, human"[Mesh] OR "respiratory tract infections"[Mesh]) AND ((((((rapid influenza test*) OR rapid testing) OR rapid diagn*) OR rapid test) OR "point-of-care testing"[Mesh]) OR point of care)) AND (((randomized controlled trial) OR trial OR "clinical trial" [Publication Type] OR "controlled clinical trial" [Publication Type])) Our search yielded a total of 598 articles. Screening of these articles and their references resulted in 10 relevant trials. An overview of the characteristics and results of these trials is included in Table 1.

\section{General practice}

We found no randomized trials on the effect of influenza POC tests on any of the clinically relevant outcome measures in general practice.

\section{Emergency department}

Adults

In 2017, a UK trial investigated, over two winter seasons, the added value of routinely administering a POC molecular test for several respiratory viruses (including influenza A and $\mathrm{B}$ ) to patients presenting with acute respiratory illness or a body temperature $>37.5^{\circ} \mathrm{C}$ at an emergency department or acute admissions unit. ${ }^{12}$ Routine use of the POC test had no effect on the number of patients treated with antibiotics compared to routine care, but led to more correct use of antivirals in hospitalized influenza-positive patients (91 vs 65\%; difference: $26 \%$ (95\% Cl 9.6-43.2)) and to shorter hospitalization (5.7 vs 6.8 days; 
difference: -1 (95\% Cl-2.2 to -0.3)). Overall, the number of patients receiving antivirals did not differ between the intervention and control groups (18 vs. 14\%; difference: 4\% (95\% Cl -1.5 to 9.4)). These results should be interpreted with caution, as patients were not tested for influenza alone.

Previous randomized trials published in the period 2003-2016 showed that influenza POC tests did not have a statistically significant and clinically relevant effect on our chosen outcome measures (Table 1). In England, a large trial is currently investigating the effect of POC PCRbased influenza tests on the correct use of antivirals in influenza-positive patients, length of hospitalization, antibiotic use and isolation measures.

\section{Children}

The majority of trials were conducted among children $<5$ years old visiting the emergency department (Table 1). A meta-analysis of RCTs showed that additional investigations in the form of chest $\mathrm{X}$-rays, routine blood tests or blood cultures could be avoided in approximately $20 \%$ of the children. The use of influenza POC tests had no effect on the length of hospitalization or the use of antibiotics.

\section{Observational studies}

The aforementioned meta-analysis showed that, in four out of five observational studies, the use of influenza POC tests led to a reduction in antibiotic use..$^{13}$ The difference in results between the observational studies and randomized trials may be attributable to bias and differences in the number of patients who had influenza and were prescribed antibiotics at the start of the studies. It is not possible to draw any conclusions on the added value of influenza POC tests on the basis of this. A recent systematic review found that, in the majority of included observational studies, influenza POC tests led to a decrease in the length of stay at the emergency department. ${ }^{14}$ Due to the varying results of the individual studies, no definite conclusion could be drawn. The results may have varied because not only does testing at the point-of-care affect the length of hospitalization, but also bed availability, discharge procedures and the efficiency of healthcare providers.

A Dutch retrospective observational study showed that a POC PCR-based influenza test was as accurate as a regular PCR-test for influenza. ${ }^{26}$ The length of stay at the emergency department of influenza-positive patients was 12 minutes shorter in the relatively heavy influenza season that the POC test was used (2017/2018) compared to the previous season (2016/2017) ( $p=$ 0.03). In the $2017 / 2018$ influenza season, $73 \%$ of the influenza-positive patients were admitted, compared to $91 \%$ in the previous year $(p=0.00)$. Thus unnecessary isolation could be avoided and patient throughput was improved. The throughput was facilitated by a temporary admission ward for influenza-positive patients, using beds from other departments. 


\section{Implementation}

For the successful implementation of a diagnostic test, not only its analytical accuracy and clinical efficacy are important, but also other factors such as the practicality and cost-effectiveness of the test, the interpretation of the test results by the user, and the level of stress experienced by patients undergoing the test.

Influenza POC tests based on antigen detection or PCR can be easily applied to body material collected by means of a nasopharyngeal swab. However, POC PCR-based influenza tests require the support and involvement of laboratory staff trained in molecular diagnosis; this is currently only feasible with direct support from a medical laboratory. A cost-effectiveness study using a hypothetical cohort of elderly people with influenza during the peak influenza season in Hong Kong found that a POC molecular influenza test was cost-effective. A clinical trial-based cost-effectiveness analysis in the UK showed that a POC PCR-based influenza test was not cost-effective.

There is modest interest in the use of influenza POC tests in primary care (36\% of Dutch GPs and 59\% of Belgian GPs are interested in using rapid influenza tests in primary care). Due to the reasons mentioned above, only POC tests using antigen detection are currently being considered. However, the analytical accuracy of such POC tests needs to be improved before further research into their effect on clinically relevant outcome measures or their costeffectiveness can be meaningful.

An influenza POC test with a short analysis time (preferably $<15$ minutes) is desirable to ensure that the result can guide clinical decision-making. In addition to the influenza virus, there are many other agents that can cause influenza-like illness. It is important to be able to exclude influenza infection reliably, given the risk of a severe course, the high influenza prevalence and the associated high disease burden. POC tests are available that can differentiate between multiple - often viral - pathogens at the same time. This specific distinction is of minor importance, because the clinical picture - even after ruling out an influenza infection - should inform the choice of treatment and compliance with existing guidelines.

\section{DISCUSSION}

\section{General practice}

From a practical point of view, only the influenza POC tests that use antigen detection are currently being considered for general practice. However, these are not sufficiently accurate to exclude influenza infection. Furthermore, the test result is not available within the average consultation time, there is no clear need for rapid influenza tests in general practice, and lastly, there is no evidence of the clinical efficacy of such tests in primary care. 
Table 1. Trials in which influenza POC tests were evaluated on: length of stay on the emergency department, length of hospitalization, mortality, antibiotic prescriptions or antiviral drug prescriptions.

\begin{tabular}{llllll}
\hline Authors & Setting & Inclusion criteria & $\begin{array}{l}\text { Study } \\
\text { size }\end{array}$ & $\begin{array}{l}\text { Intervention } \\
\text { (technique POCT, } \\
\text { testing-time) }\end{array}$ & Control \\
\hline Andrews, & Emergency & Patients $\geq 16$ & $\mathrm{n}=545$ & FilmArray $\mathbb{2}$ & Routine \\
$2017^{18}$ & department, & years with & & Respiratory Panel & diagnostics \\
& United & ILI or signs of & & polymerase chain & (laboratory- \\
& Kingdom & respiratory tract & reaction, BioFire & based PCR \\
& infection & (PCR, test-time 60 & testing)
\end{tabular}

\begin{tabular}{|c|c|c|c|c|c|}
\hline $\begin{array}{l}\text { Brendish, } \\
2017^{12}\end{array}$ & $\begin{array}{l}\text { Emergency } \\
\text { department, } \\
\text { United } \\
\text { Kingdom }\end{array}$ & $\begin{array}{l}\text { Patients } \geq 18 \\
\text { years with acute } \\
\text { respiratory } \\
\text { illness or fever } \\
\text { higher than } \\
37.5^{\circ} \mathrm{C} \text { ( } \leq 7 \text { days) }\end{array}$ & $n=720$ & $\begin{array}{l}\text { FilmArray }{ }^{\circledR} \\
\text { Respiratory Panel } \\
\text { polymerase chain } \\
\text { reaction, BioFire } \\
\text { (PCR, test-time } 60 \\
\text { min) }\end{array}$ & $\begin{array}{l}\text { Routine } \\
\text { diagnostics (not } \\
\text { specified) }\end{array}$ \\
\hline
\end{tabular}

\begin{tabular}{|c|c|c|c|c|c|}
\hline $\begin{array}{l}\text { Nicholson, } \\
2014^{16}\end{array}$ & $\begin{array}{l}\text { General and } \\
\text { University } \\
\text { hospital, } \\
\text { United } \\
\text { Kingdom }\end{array}$ & $\begin{array}{l}\text { Patients } \geq 65 \\
\text { years, patients } \\
\text { aged 18-64 years } \\
\text { if known with any } \\
\text { cardiopulmonary } \\
\text { conditions, } \\
\text { patients with } \\
\text { an acute } \\
\text { exacerbation } \\
\text { of chronic } \\
\text { cardiopulmonary } \\
\text { illness or an acute } \\
\text { cardiopulmonary } \\
\text { illness }\end{array}$ & $n=1252$ & $\begin{array}{l}\text { Group A) Quidel } \\
\text { QuickVue influenza } \\
\text { (antigendetection, } \\
\text { test-time } 10 \text { min) } \\
\text { and BinaxNOW: } \\
\text { antigendetection for } \\
\text { pneumococcal disease } \\
\text { ( } \mathrm{n}=418 \text { ) } \\
\text { Group B) PCR-tests } \\
\text { for influenza A and } \\
\text { B and } \\
\text { pneumococcal antigen } \\
\text { test in laboratory } \\
\text { (n=415) }\end{array}$ & $\begin{array}{l}\text { Group C) } \\
\text { conventional lab } \\
\text { tests; culture on } \\
\text { influenza A/B, } \\
\text { RSV A/B and S. } \\
\text { pneumoniae, } \\
\text { serology } \\
\text { influenza A/B } \\
\text { (n=419) }\end{array}$ \\
\hline
\end{tabular}




\section{Additional information* Outcome}

Combined POCT

for adenovirus,

coronavirus, human

metapneumovirus,

influenza A/B,

parainfluenza virus,

respiratory syncytial

virus, bordetella

pertussis, chlamydia

pneumoniae,

mycoplasma

pneumoniae

\section{Results}

Mortality (up to 30 days) after inclusion:

Intervention group 4\%, control group 4\%, aOR 0.9

(95\% Cl 0.3-2.2), p=0.79

Length of Median Length of hospitalization in hours:

hospitalization Intervention group 61 hours vs. control group 54,

$p=0.66$

Length stay ED Not reported

Prescriptions Total presciptions not reported

antibiotics AB use during admission:

Intervention group 75\%, control group 77\%, aOR

$1.0(95 \%$ Cl 0.6-1.5), $p=0.99$

Prescriptions Not reported

antiviral drugs

Combined POCT Mortality

for adenovirus,

coronavirus, human

Length of

metapneumovirus,

influenza A/B,

parainfluenza virus,

respiratory syncytial

virus, rhinovirus or

enterovirus hospitalization

Length stay ED

Prescriptions

antibiotics

Prescriptions

antiviral drugs

Mortality

Length of

hospitalization

Length stay ED

Prescriptions

antibiotics

Prescriptions

antiviral drugs
Mortality within 30 days: POCT 9 (3\%), control 16 (5\%), OR 0.54 (0.3-1.2), $p=0.15$

In days: intervention group 5.7 (SD 6.3), control group 6.8 (SD 7.7); $p=0.04$

Not reported

Intervention group 301 (84\%) vs. control group 294

(83\%); $p=0.84$ )

Intervention group 52 (91\%), control group (24

(65\%) ; difference 26.4\%; $p=0.003$

Mortality within 28 days after admission:

Group A) 1 (3.7\%), group B) 0 (0\%), group C) 2

(5.9\%), $p=0.64$

Difference in length of hospitalization for influenza:

In days: group A) 2.08 , group B) 1.96, group C) 3.59

Cox HR (95\% Cl; Pvalue): group A) 1.27 (0.76-2.14; $p=0.36)$, group B) 1.25 (0.76-2.04; $p=0.38)$, group

C) 1

Not reported

Not reported

Not reported 
Table 1. Continued.

\begin{tabular}{|c|c|c|c|c|c|}
\hline Authors & Setting & Inclusion criteria & $\begin{array}{l}\text { Study } \\
\text { size }\end{array}$ & $\begin{array}{l}\text { Intervention } \\
\text { (technique POCT, } \\
\text { testing-time) }\end{array}$ & Control \\
\hline $\begin{array}{l}\text { Doan, } \\
2009^{19}\end{array}$ & $\begin{array}{l}\text { Emergency } \\
\text { department } \\
\text { in Children's } \\
\text { hospital, } \\
\text { Canada }\end{array}$ & $\begin{array}{l}\text { Children aged } \\
2 \text { - } 24 \text { months } \\
\text { with fever and } \\
\text { acute respiratory } \\
\text { tract infection }\end{array}$ & $n=204$ & $\begin{array}{l}\text { direct immuno- } \\
\text { fluorescence } \\
\text { assay (Chemicon } \\
\text { International) for: } \\
\text { Adenovirus, Influenza } \\
\text { virus A/B and } \\
\text { parainfluenzavirus 1, } \\
\text { 2, } 3 \text { and respiratory } \\
\text { syncytial virus } \\
\text { (immunofluorescence, } \\
\text { test-time 30-150min) }\end{array}$ & $\begin{array}{l}\text { Standard care } \\
\text { (not specified) }\end{array}$ \\
\hline $\begin{array}{l}\text { Cohen, } \\
200720\end{array}$ & $\begin{array}{l}\text { Pediatricians } \\
\text { working in } \\
\text { outpatient } \\
\text { clinics, France }\end{array}$ & $\begin{array}{l}\text { Children aged }>1 \\
\text { year with fever } \\
\text { (<48 hours) and } \\
\text { ILI }\end{array}$ & $n=602$ & $\begin{array}{l}\text { Quidel QuickVue } \\
\text { (antigendetection, } \\
\text { test-time } 10 \mathrm{~min} \text { ) }\end{array}$ & $\begin{array}{l}\text { Standard care } \\
\text { (not specified) }\end{array}$ \\
\hline
\end{tabular}

\begin{tabular}{|c|c|c|c|c|c|}
\hline $\begin{array}{l}\text { Iyer, } 2006 \\
21\end{array}$ & $\begin{array}{l}\text { Emergency } \\
\text { department } \\
\text { in Children's } \\
\text { hospital, } \\
\text { United States }\end{array}$ & $\begin{array}{l}\text { Patients aged } \\
2-3 \text { months with } \\
\text { temperature } \\
\geq 38^{\circ} \mathrm{C} \text { and } \\
\text { patients aged } \\
\text { 3-24 months } \\
\text { with } \geq 39^{\circ} \mathrm{C}\end{array}$ & $n=700$ & $\begin{array}{l}\text { Quidel QuickVue } \\
\text { Influenza } \\
\text { (antigendetection, } \\
\text { test-time } 10 \mathrm{~min} \text { ) }\end{array}$ & $\begin{array}{l}\text { Standard care } \\
\text { (not specified) }\end{array}$ \\
\hline
\end{tabular}


Mortality

Length of

hospitalization

Length stay ED

Prescriptions

antibiotics

Prescriptions

antiviral drugs

Cluster randomization

between various

pediatric clinics in

France, 16 pediatricians

received point-of-care tests, 14 pediatricians did not

Mortality

Length of

hospitalization

Length stay ED

Prescriptions antibiotics

Prescriptions antiviral drugs

Mortality

Length of hospitalization

Length stay ED

Prescriptions antibiotics

Prescriptions antiviral drugs
Not reported

Not reported

Intervention group 105.7 min. (SD 188.04), control group $156.1 \mathrm{~min}$ (SD 235.82), mean difference -50.4 (95\% Cl -104.6 to 3.7), P value not reported. Intervention group 16 (18\%), control group 23 (20.9\%), RR 0.86 (95\% Cl; 0.48-1.53), P value not reported

Not reported

\section{Not reported}

Does not apply (outpatient clinic)

Does not apply (outpatient clinic)

Intervention group 33 (9.5\%), control group 10

(3.9\%), $p=0.008$

Intervention group 131 (37.9\%), 35 (13.7\%), $p<0.0001$

Not reported

Length of hospitalization not reported

OR for hospital admission: Intervention group aOR 0.56, control group aOR 0.53, $\mathrm{p}=0.72$

POCT test positive $=183 \mathrm{~min}$ (SE $7 \mathrm{~min}$ ), POCT test negative $=213 \mathrm{~min}$ (SE 6min), Control group with positive test $=198$ (SE 9min), control group with negative test $=207$ (SE 6min), $p=0.03$

Intervention group $n=54$ (15.7\%), control group $\mathrm{n}=59$, aOR (intervention group) 0.53, aOR (control group) $0.57, p=0.70$

Not reported 
Table 1. Continued.

\begin{tabular}{|c|c|c|c|c|c|}
\hline Authors & Setting & Inclusion criteria & $\begin{array}{l}\text { Study } \\
\text { size }\end{array}$ & $\begin{array}{l}\text { Intervention } \\
\text { (technique POCT, } \\
\text { testing-time) }\end{array}$ & Control \\
\hline $\begin{array}{l}\text { Poehling, } \\
2006^{22}\end{array}$ & $\begin{array}{l}\text { Emergency } \\
\text { department } \\
\text { in Children's } \\
\text { hospital, } \\
\text { United States }\end{array}$ & $\begin{array}{l}\text { Children aged } \\
\text { younger than } \\
5 \text { years with } \\
\text { fever or acute } \\
\text { respiratory } \\
\text { symptoms }\end{array}$ & $n=468$ & $\begin{array}{l}\text { Quidel } \\
\text { QuickVue Influenza } \\
\text { (antigendetection, } \\
\text { test-time not } \\
\text { reported) }\end{array}$ & $\begin{array}{l}\text { Viral culture or } \\
\text { PCR-analysis }\end{array}$ \\
\hline
\end{tabular}

\begin{tabular}{|c|c|c|c|c|c|}
\hline $\begin{array}{l}\text { Abanses, } \\
2006^{23}\end{array}$ & $\begin{array}{l}\text { Emergency } \\
\text { department } \\
\text { in Children's } \\
\text { hospital, } \\
\text { United States }\end{array}$ & $\begin{array}{l}\text { Children aged } \\
\text { 3-36 months } \\
\text { with fever }\end{array}$ & $n=1007$ & $\begin{array}{l}\text { Directigen Flu A/B } \\
\text { test } \\
\text { (antigendetection, } \\
\text { test-time not } \\
\text { reported) }\end{array}$ & $\begin{array}{l}\text { Standard care } \\
\text { (not specified) }\end{array}$ \\
\hline
\end{tabular}

\begin{tabular}{|c|c|c|c|c|c|}
\hline $\begin{array}{l}\text { Bonner, } \\
2003^{24}\end{array}$ & $\begin{array}{l}\text { Emergency } \\
\text { department } \\
\text { in Children's } \\
\text { hospital, } \\
\text { United States }\end{array}$ & $\begin{array}{l}\text { Children aged } \\
2 \text { months to } 21 \\
\text { years with ILI }\end{array}$ & $\mathrm{n}=418$ & $\begin{array}{l}\text { FluOIA test for } \\
\text { influenza A/B } \\
\text { (antigendetection, } \\
\text { test-time 20-25 min) }\end{array}$ & $\begin{array}{l}\text { Standard care } \\
\text { (not specified) }\end{array}$ \\
\hline
\end{tabular}




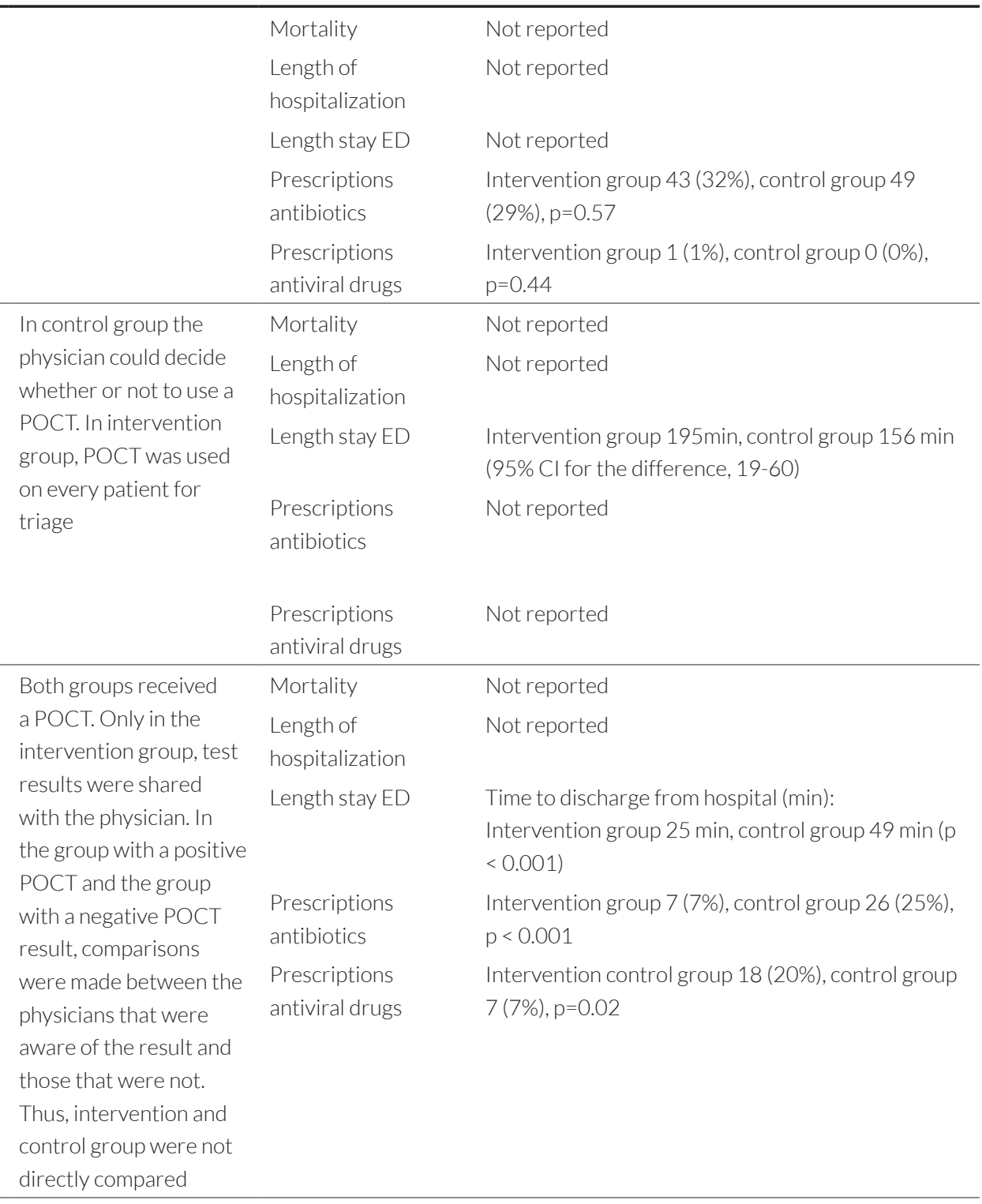


Table 1. Continued.

\begin{tabular}{|c|c|c|c|c|c|}
\hline Authors & Setting & Inclusion criteria & $\begin{array}{l}\text { Study } \\
\text { size }\end{array}$ & $\begin{array}{l}\text { Intervention } \\
\text { (technique POCT, } \\
\text { testing-time) }\end{array}$ & Control \\
\hline $\begin{array}{l}\text { Esposito, } \\
2003^{25}\end{array}$ & $\begin{array}{l}\text { Emergency } \\
\text { department } \\
\text { in Children's } \\
\text { hospital, Italy }\end{array}$ & Children with ILI & $n=957$ & $\begin{array}{l}\text { Quickvue influenza } \\
\text { A/B } \\
\text { (antigendetection, } \\
\text { test-time } 10 \mathrm{~min} \text { ) }\end{array}$ & Not specified \\
\hline
\end{tabular}

* In all studies, the physician could run other diagnostic tests besides the POCT if he/she thought this was needed.

Emergency department

POC PCR-based influenza tests, on the other hand, are accurate, rapid and easy to use at the emergency department. However, there is little direct evidence of any effect of influenza POC tests on the use of antibiotics and antivirals, length of stay at the emergency department, and length of hospitalization. Before such rapid influenza tests can be widely used in emergency departments, it would be scientifically desirable to replicate the promising results of the aforementioned English trial specifically for a rapid influenza test and to conduct a thorough cost-effectiveness study. Influenza POC tests can reduce the length of stay at an emergency department and may improve patient throughput in hospital, provided no capacity problems exist. These findings, which are important for patient care and the care chain, are partially supported by field studies. 
Patients with a positive

POCT are compared with those with a negative POCT and a

control group without

POCT respectively

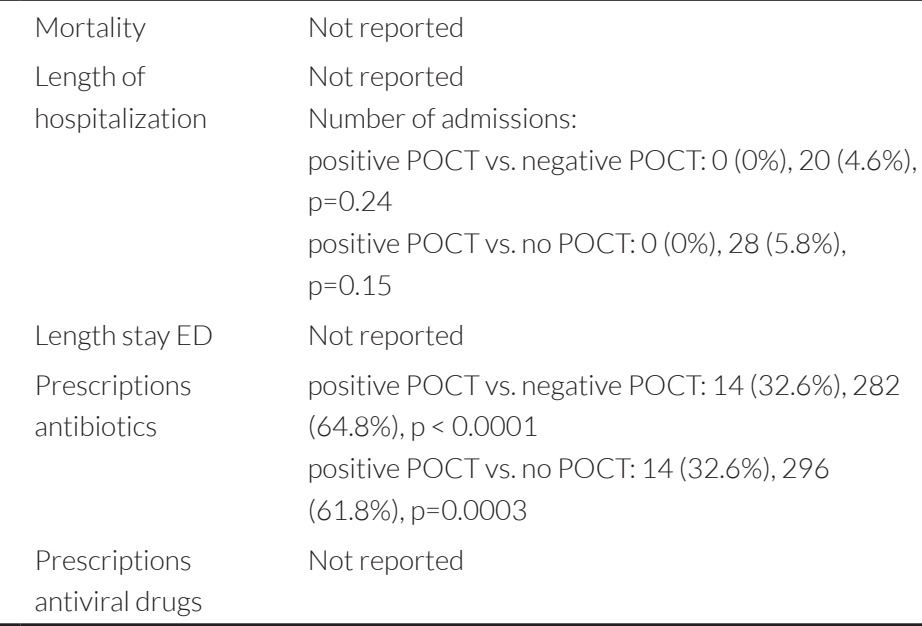

(n)

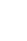

Abbreviations; AB: antibiotics, AVD: antiviral drugs, ED: emergency department, ILI: influenza-like illness, aOR: adjusted Odds Ratio, OR: Odds Ratio, RR: relative risk, POCT: point-of-care test.

\section{CONCLUSION}

The current literature warrants the use of POC PCR-based influenza tests at emergency departments as long as rapid diagnostics are not used at the expense of careful diagnostics. Implementation of influenza POC tests in general practice is, for the time being, a bridge too far. 


\section{REFERENCES}

1. Sauro A, Barone F, Blasio G, Russo L, Santillo L. Do influenza and acute respiratory infective diseases weigh heavily on general practitioners' daily practice? Eur J Gen Pract. 2006;12:34-6.

2. Beysard N, Yersin B, Meylan P, Hugli O, Carron PN. Impact of the 2014-2015 influenza season on the activity of an academic emergency department. Intern Emerg Med. 2018;13:251-6.

3. Schols AMR, Dinant GJ, Hopstaken R, Price CP, Kusters R, Cals JWL. International definition of a pointof-care test in family practice: a modified e-Delphi procedure. Fam Pract. 2018;35:475-80.

4. Centers for Disease Control and Prevention. Guide for considering influenza testing when influenza viruses are circulating in the Community [cited January 2019]. Available from: www.cdc.gov/flu/ professionals/diagnosis/consider-influenza-testing.htm

5. Lankelma JM, Hermans MHA, Hazenberg EHLCM, et al. Implementation of point-of-care testing and a temporary influenza ward in a Dutch hospital. Neth J Med. 2019;77:109-15 Medline.

6. Basile K, Kok J, Dwyer DE. Point-of-care diagnostics for respiratory viral infections. Expert Rev Mol Diagn. 2018;18:75-83.

7. Chen L, Tian Y, Chen S, Liesenfeld O. Performance of the Cobas( $(\mathbb{B})$ Influenza A/B assay for rapid PCRbased detection of influenza compared to Prodesse ProFlu+ and viral culture. Eur J Microbiol Immunol (Bp). 2015;5:236-45.

8. Hassan F, Crawford J, Bonner AB, Ledeboer NA, Selvarangan R. Multicenter evaluation of the Alere ${ }^{\mathrm{TM}}$ i influenza A\&B assay using respiratory specimens collected in viral transport media. Diagn Microbiol Infect Dis. 2018;92:294-8.

9. Schellekens JJA, Willemse HFM, Sterks M, Lutgens SPM, Hermans MHA. Evaluation of the Cobas ${ }^{\circ}$

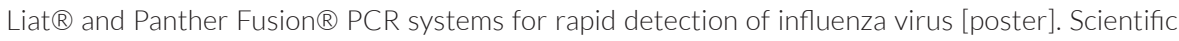
Spring Meeting KNVM \& NVMM; 2018.

10. Bruning AHL, de Kruijf WB, van Weert HCPM, et al. Diagnostic performance and clinical feasibility of a point-of-care test for respiratory viral infections in primary health care. Fam Pract. 2017;34:558-63.

11. Mese S, Akan H, Badur S, Uyanik A; Istanbul Rapid Test Study Group. Analytical performance of the $B D$ veritor ${ }^{\mathrm{TM}}$ system for rapid detection of influenza virus $A$ and $B$ in a primary healthcare setting. BMC Infect Dis. 2016;16:481.

12. Brendish NJ, Malachira AK, Armstrong L, et al. Routine molecular point-of-care testing for respiratory viruses in adults presenting to hospital with acute respiratory illness (ResPOC): a pragmatic, openlabel, randomised controlled trial. Lancet Respir Med. 2017;5:401-11.

13. Lee JJ, Verbakel JY, Goyder CR, Ananthakumar T, Tan PS, Turner PJ, et al. The clinical utility of pointof-care tests for influenza in ambulatory care: A systematic review and meta-analysis. Clin Infect Dis. 2018.

14. Egilmezer E, Walker GJ, Bakthavathsalam P, et al. Systematic review of the impact of point-of-care testing for influenza on the outcomes of patients with acute respiratory tract infection. Rev Med Virol. 2018;28:e1995.

15. You JHS, Tam LP, Lee NLS. Cost-effectiveness of molecular point-of-care testing for influenza viruses in elderly patients at ambulatory care setting. PLoS One. 2017;12:e0182091.

16. Nicholson KG, Abrams KR, Batham S, et al. Randomised controlled trial and health economic evaluation of the impact of diagnostic testing for influenza, respiratory syncytial virus and Streptococcus pneumoniae infection on the management of acute admissions in the elderly and high-risk 18- to 64-year-olds. Health Technol Assess. 2014;18:1-274, 
17. Howick J, Cals JWL, Jones C, et al. Current and future use of point-of-care tests in primary care: an international survey in Australia, Belgium, The Netherlands, the UK and the USA. BMJ Open. 2014;4:e005611.

18. Andrews D, Chetty Y, Cooper BS, et al. Multiplex PCR point of care testing versus routine, laboratorybased testing in the treatment of adults with respiratory tract infections: a quasi-randomised study assessing impact on length of stay and antimicrobial use. BMC Infect Dis. 2017;17:671.

19. Doan $\mathrm{QH}$, Kissoon N, Dobson S, et al. A randomized, controlled trial of the impact of early and rapid diagnosis of viral infections in children brought to an emergency department with febrile respiratory tract illnesses. J Pediatr. 2009;154:91-5.

20. Cohen R, Thollot F, Lécuyer A, et al. [Impact of the rapid diagnosis downtown in the assumption of responsibility of the children in period of influenza]. Arch Pediatr. 2007;14:926-31.

21. Iyer SB, Gerber MA, Pomerantz WJ, Mortensen JE, Ruddy RM. Effect of point-of-care influenza testing on management of febrile children. Acad Emerg Med. 2006;13:1259-68.

22. Poehling KA, Zhu Y, Tang YW, Edwards K. Accuracy and impact of a point-of-care rapid influenza test in young children with respiratory illnesses. Arch Pediatr Adolesc Med. 2006;160:713-8.

23. Abanses JC, Dowd MD, Simon SD, Sharma V. Impact of rapid influenza testing at triage on management of febrile infants and young children. Pediatr Emerg Care. 2006;22:145-9.

24. Bonner AB, Monroe KW, Talley LI, Klasner AE, Kimberlin DW. Impact of the rapid diagnosis of influenza on physician decision-making and patient management in the pediatric emergency department: results of a randomized, prospective, controlled trial. Pediatrics. 2003;112:363- 7.

25. Esposito S, Marchisio P, Morelli P, Crovari P, Principi N. Effect of a rapid influenza diagnosis. Arch Dis Child. 2003;88:525-6.

26. Dautzenberg P, Macken T, van Gageldonk-Lafeber R, Lutgens S. Griepgolf onder controle met ziekenhuisbrede aanpak. Med Contact. 2018;44:18-20. 


\section{SUPPLEMENTARY INFORMATION}

Supplement 1. Example of how the prevalence of influenza can influence the analytical accuracy of a POC test.

In this example the sensitivity of the test is $80 \%$, the specificity is $90 \%$.

With a decreasing prevalence of influenza, the positive predictive value drops.

A) influenza prevalence $20 \%$

\begin{tabular}{llll}
\hline & influenza & no influenza & \\
\hline POC test + & 16 & 8 & $P P V=16 / 24=0.67$ \\
POC test - & 4 & 72 & $N P V=72 / 76=0.95$ \\
& 20 & 80 & 100 \\
\hline
\end{tabular}

B) influenza prevalence 10\%

\begin{tabular}{llll}
\hline & influenza & no influenza & \\
\hline POC test + & 8 & 9 & $P P V=8 / 17=0.47$ \\
POC test - & 2 & 81 & $N P V=81 / 83=0.98$ \\
& 10 & 90 & 100 \\
\hline
\end{tabular}

$\mathrm{PPV}=$ positive predicting value

NPV = negative predicting value

POC test: point-of-care test 


Chapter 7

Summary and general discussion 



\section{SUMMARY AND GENERAL DISCUSSION}

The general aim of this thesis is to contribute to the evidence base for reducing the impact of influenza in primary care. We chose both quantitative and qualitative research methods together with reflections of the existing evidence to achieve a multidimensional and good understanding of how to move onwards in the field of influenza vaccination and research.

First, in a narrative review, we performed an ethical and methodological analysis showing important barriers that would hinder a new RCT on influenza vaccination in the elderly with hospitalization or mortality as an endpoint. Secondly, by conducting surveys among approximately 500 general practitioners (GPs) in different regions in the Netherlands, we examined the knowledge, attitudes and beliefs (KABs) of GPs towards influenza vaccination. Thirdly, we assessed the long-term outcome of influenza vaccination on mortality in the elderly by conducting a 25-year follow-up study of an RCT on the efficacy of influenza vaccination at baseline. Next, we explored the relation between the antibody response following quadrivalent influenza vaccination and long-term mortality in the elderly. Lastly, in a narrative review, we evaluated the analytical accuracy and added value of influenza point-of-care tests in the general practice and emergency department (ED).

To explore the relation between vaccination and antibody response respectively to mortality, we used a large trial that evaluated the effect of influenza vaccination in the elderly during the winter of 1991/1992. ${ }^{1}$ This trial was conducted at our Department of Family Medicine in Maastricht, largely by the same research team that supported the work for this thesis. We completed the trial data with mortality statistics creating a follow-up study to address some of the questions previously mentioned.

In this final chapter, I will first focus on our main findings and relate these findings to contemporary literature. Furthermore I will discuss the methodological considerations of performing a cohort study based on a randomized trial. Finally the implications of our studies are discussed, making recommendations for future research.

\section{Relating our main findings to contemporary evidence}

Barriers for an influenza vaccination trial in the elderly with mortality as an endpoint

In chapter 2, we performed an ethical and methodological analysis showing important barriers that would hinder a new RCT on influenza vaccination in the elderly with hospitalization or mortality as an endpoint. ${ }^{2}$ Based on the lack of direct evidence for an effect of influenza vaccination on these endpoints and contradictory results of large observational studies, the scientific urge to increase the existing knowledge by conducting a placebo-controlled trial on the effects of influenza vaccination in the elderly on mortality is understandable. However, we cannot escape from critically reviewing the ethical and scientific considerations that pose a threat to the acceptability of such a trial, and for various reasons, a new placebo-controlled trial evaluating the effects of mortality in the elderly is both ethically and methodologically 
problematic. An important condition in the justification of a trial is the existence of genuine uncertainty in regard to the answer to a research question. Therefore an extensive analysis of the existing levels of knowledge is needed to support conclusions on the effect of vaccination on mortality. Even if a so called 'clinical equipoise' status applies, denying a control group vaccination would be problematic because vaccination is considered 'competent care' and withholding vaccination could substantially increase patients' risk for influenza and its complications. Given the high burden of disease and already proven benefits of vaccination, such a trial is unlikely to meet the Declaration of Helsinki stating that the importance of a trial must outweigh the risk patients are exposed to. Moreover, while a placebo-controlled trial in vaccine refusers may be considered, such a trial is unlikely to meet substantial methodological barriers regarding trial size and generalizability, as we will discuss later on in this general discussion (methodology). We conclude that a new trial is unlikely to provide for a direct answer, let alone change current policy. Although head-to-head trials between new (types of) influenza vaccines and the regular vaccine have no large ethical constraints, unrealistically high numbers of participants should be included to meet the sample size required to prove superiority of a new vaccine on mortality as an endpoint, making them methodologically (and practically) difficult to conduct.

Our essay was the first scientific paper explicitly reviewing the ethical and methodological pro's and con's for conducting an RCT for mortality as an endpoint. We openly invited researchers considering such trials to address the ethical challenges discussed in our manuscript. In response to publication of our work, a team of influenza experts published a commentary on our work. ${ }^{3}$ Interestingly, these experts state that an RCT evaluating the effects of influenza vaccination on mortality/hospitalization in the elderly should be possible, both from ethical and methodological point of view. They conclude that based on the available RCTs, the effect of influenza vaccination in preventing influenza in elderly is not sufficiently proven. Suggesting that influenza vaccination may not reduce complications caused by influenza, that other infections may occur more frequently in the vaccinated group and emphasising that vaccine effectiveness strongly varies over the various years, they consider influenza vaccination not to be cost efficient. Therefore, they state that the expenses for the influenza vaccine programme (60 million euro's in the Netherlands, 1.7 billion USD in the US) can easily be saved in the future if a trial would prove the inefficacy of influenza vaccination. In these authors' view, denying patients influenza vaccination for the sake of a new RCT should not increase their health-risks because of a potential increase in incidence of infections other than influenza after vaccination and a potential waning effect of influenza vaccination after repetitive influenza vaccination over the years. However, the RCT conducted by our research group contradicts the statement that the total amount of ILI-cases would be higher in vaccinated groups; the absolute amount of influenza-like illness (ILI) cases was actually smaller in the vaccinated group than in the unvaccinated group, although differences (in favor of the vaccinated group) were not statistically significant. ${ }^{1}$ The authors' statement seems to be based on an observational study suggesting that vaccination reduces the number of influenza virus infections but not the overall number of ILI episodes; i.a. other pathogens 
fill the gap. ${ }^{4}$ However, the same study showed that influenza vaccination significantly reduced influenza virus infection by $73 \%$. Since influenza is known to cause more complications in elderly than other respiratory tract infections, ${ }^{5}$ it remains relevant to reduce the proportion of influenza in these ILIs, even if the total amount of ILIs would stay the same. Also, when it comes to the expenses of the influenza vaccination programme, the societal costs of influenza should also be taken in consideration. These costs have been estimated to be up to 10-20 times higher than the costs of the influenza vaccination programme. ${ }^{6}$ Up to our knowledge, after previous preliminary discussion, ${ }^{7}$ this is the first time that the pros and cons from both ethical and methodological points of view of an influenza trial are fully discussed in literature. Avoidable waste of money in biomedical research is huge; it is estimated that about $85 \%$ of research investment - equating to $\$ 200$ billion of the investment in 2010 - is wasted. ${ }^{8}$ We believe that the scientific community has a shared responsibility in contributing to good quality research and care and as such should try to align when it comes to complicated research questions. Therefore, we think research will profit from correspondences as these.

\section{Attitudes and beliefs of GPs in the Netherlands regarding influenza vaccination}

In chapter 2 we have seen that the efficacy of influenza vaccination in the elderly has long been a topic of debate, mainly due to the lack of direct RCT-based evidence on effects of influenza vaccination on severe morbidity and mortality in the elderly. In the Netherlands, GPs have always been key actors in implementing influenza vaccination. In chapter $\mathbf{3}$, we examined the knowledge, attitudes and beliefs of GPs towards influenza vaccination in different regions in the Netherlands before and during the 2018/2019 influenza epidemic. ${ }^{9}$ One of the important findings is that the lack of direct evidence on the effect of influenza vaccination on mortality also bothered a wide group of Dutch GPs. Our study showed that the majority of $60.5 \%$ desires a trial evaluating the effect of influenza vaccination on hospitalization and/or mortality in the elderly. Moreover, we found that $71.9 \%$ of the GPs receive influenza vaccination and 4 out of 5 recommend influenza vaccination for their practice personnel. This representative study was large enough to draw conclusions based on these findings, thus to compare these results with other (inter)national studies. Latest studies performed in the Netherlands reported a coverage rate in GPs of 36\% for seasonal influenza vaccination in 2007/2008 ${ }^{10}$ and even up to 63\% during pandemic seasons (2009/2010, Mexican fever). ${ }^{11}$ A potential explanation for the increase in coverage rate, could be the gradual implementation of the recommendations for healthcare workers (HCWs) to be vaccinated against influenza. ${ }^{12}$

In our study, most frequently reported motives in GPs for receiving influenza vaccination were personal protection against influenza and lowering of the risk of transmitting influenza to patients, whereas having no medical indication for influenza vaccination and the conviction that one is already protected against influenza were the most common reasons for not being vaccinated. These motives do not seem to have changed over the past decade ${ }^{10}$ and apply internationally. ${ }^{13}$ Similar to our study, concerns about side effects, forgetfulness, and doubts about the vaccine's efficacy are less frequently 
reported motives (ranging from 2.5-28.6\%) for non-vaccination in GPs. ${ }^{13}$ Besides all quantitative data, qualitative data showed that also the belief that influenza is not a serious illness is a potential barrier for HCW vaccination. ${ }^{14}$ Our study clarifies that - unlike in patients with a medical indication - the majority of GPs do not agree that the severity of symptoms and the severity and chance of complications of influenza in healthy elderly are that relevant that this would require prevention by means of an effective vaccine.

To our best knowledge, this is the first study to examine the attitudes of GPs towards mandatory influenza vaccination in practice personnel. Whereas only $41.2 \%$ of the GPs in our study supports mandatory vaccination of practice personnel with direct patient contact, Desante et al. showed that in the United States, 84\% of the physicians working in internal medicine or emergency medicine supported mandatory influenza vaccination among HCW. ${ }^{15}$ Differences in the severity of complications caused by influenza in patients presenting in this setting compared to primary care may partly explain these differences.

As a GP, my personal experience supports our study findings that GPs do question the need of influenza vaccination in healthy elderly and the majority desires a large new trial evaluating the effect of influenza vaccination. When I inform GPs about my PhD study, they often express their concerns when it comes to the efficacy of the vaccine. Rarely different endpoints (morbidity or mortality) are discriminated or existing evidence is referred at. Often, columns written by some well known influenza criticisers in popular Dutch medical journals are mentioned. ${ }^{16}$ This emphasizes that often opinions are based on others', which might be explained from the fact that family medicine covers such a wide area and family physicians simply do not have the time (or interest) to dive into original research papers themselves. Many GPs do seem to know that seasonal mismatch between circulating strains and strains covered by the vaccine may cause reduced influenza vaccine efficacy. Therefore, GPs rarely seem to question the need of vaccination as such or in general. It is important to stress that although many GPs are somehow sceptical about influenza vaccination, in daily practice this hesitancy does not seem to translate into resistance when it comes to inviting people for their annual flu shot. This may be due to our guideline adherence and potentially also the financial compensations that GPs receive for every individual vaccinated. Since the viewpoints of influenza vaccination seem to change overtime, it would be interesting to see how attitudes and beliefs of GPs on influenza vaccination have changed during the current SARS-CoV-2 pandemic.

\section{Evidence for long-term effects of a single influenza vaccination on mortality in the elderly}

In chapter 3 we reported about the knowledge, attitudes and beliefs of GPs towards influenza vaccination showing that a majority of GPs desired for an influenza vaccination trial with mortality/ hospitalization as an endpoint. Also, 86.1\% would have no objections if their elderly patients would participate in such a trial, exposing them to the harmful risks of influenza. This (uninformed) opinion has no direct implications for the justification and feasibility of such a trial. However it should be seen as an important sign for the scientific community to clearly communicate on the state of evidence on the effects of influenza vaccination on morbidity and mortality ${ }^{17}$ in the elderly. 
Following our previous conclusions on the ethical, methodological and practical barriers of a placebo-controlled trial with severe complications and mortality as outcomes (chapter 2), along with the strong desire that lives in GPs for direct evidence on this outcome (chapter 3) we have focused on alternative ways of bringing this discussion forward. One of these intended ways is discussed in chapter 4. In this chapter we present our study in which we assessed the long-term outcome of influenza vaccination on mortality in the elderly by conducting a 25-year follow-up study of an RCT on the efficacy of influenza vaccination at baseline. ${ }^{18}$

In this study we could not demonstrate a relation between one single influenza vaccination and mortality or specific causes of death in the elderly after 25 years of follow- up: single influenza vaccination did not reduce all-cause mortality when compared to placebo (adjusted HR 0.95, 95\% Cl 0.85-1.05). Also no effect of vaccination on seasonal mortality was shown. However, although not statistically significant, survival was in favor of the vaccine group during the entire length of follow-up, with hazard rates being constant over time. In addition, subgroup analyses suggested that the effects of vaccination on mortality could be more prominent in those aged 60-64 years (adjusted HR 0.86, 95\% Cl 0.72-1.03). This is supported by literature stating that influenza-specific humoral immunity and clinical effectiveness of influenza vaccination are negatively influenced by age. ${ }^{19-21}$ Analyzing the younger elderly as such is relevant given the ongoing debate on the influence of age on vaccination effectiveness. Moreover, influenzaassociated mortality and hospitalization are substantial also in this group. ${ }^{22}$ Our finding of a statistically significant difference in median survival, i.e. 20.1 months ( $95 \% \mathrm{Cl} 2.4-37.9$ ), could be considered additional supportive evidence, not as proof of efficacy in this group.

Since similar vaccine components as used in the 1991 trial showed to provide protection against some of the influenza strains that circulated the five subsequent years after trial intervention, this might have had implications for the long-term efficacy of the trial vaccination. For instance, it has been shown that influenza vaccines can elicit immune memory and protect against drift variants or non-homologous strains. ${ }^{23,} 24$ Also, McLean et al. demonstrated that protection against influenza could also be elicited by vaccination during the previous season. This residual protection even occurred despite that the predominant viruses were antigenically distinct from previous season vaccine components. ${ }^{25}$ It should be stated that these studies included mainly young(er) individuals and thus might not fully apply to elderly due to their diminished immune responses. ${ }^{26}$ Taking into account these findings in literature, the methodological strengths (see later) and relatively low study power, in our view a protective effect of a single vaccination on long-term mortality in the youngest age group cannot be ruled out.

It is difficult to compare our study results with literature since studies predominantly evaluate seasonal effects of vaccination on the short term. In their RCT, Praditsuwan et al. evaluated mortality after one year. ${ }^{27}$ Whereas underpowered for this secondary endpoint, they could not demonstrate a relation between vaccination and mortality. A cohort study showed that vaccination was associated with lower mortality in community-dwelling elderly during six 
months of follow-up, but did not prevent death in the subgroup of healthy elderly (relative risk 0.87, 95\% Cl 0.62-1.20). ${ }^{28}$ However, in that study vaccination did reduce mortality in elderly with comorbidity (relative risk 0.67, 95\% Cl 0.48-0.94). Moreover, a meta-analysis evaluating the effect of (different types of) influenza vaccination in a diverse population (various ages, largely elderly known with cardiovascular comorbidity), showed that influenza vaccination may reduce combined cardiovascular mortality. ${ }^{29}$ Our study did not demonstrate such a comorbidityrelated subgroup effect, but may be difficult to compare with these studies since medical problems registered in our 1991 trial did not require vaccination according to the physician and thus might have been of minor clinical severity.

Since our trial was found to be the only one of the three previously mentioned RCTs that could be evaluated on long-term mortality and new placebo- controlled trials are unlikely to be approved by ethical boards, the results from our study might remain the only direct evidence available on long-term effects of influenza vaccination on mortality in community-dwelling elderly.

\section{Relation between immune response to influenza vaccination and mortality}

Given the uniqueness of the long-term follow-up of the RCT conducted at our Department of Family Medicine in 1991 (chapter 4), we used the same data for a more explorative study that aimed to answer the question whether besides the effect of vaccination as such, a higher immune response itself elicited by vaccination might have a predictive value related to better long-term outcome. As discussed in chapter 5, we hypothesized that the humoral immune response elicited by influenza vaccination in elderly might be an indicator of the immune status c.q. immunologic resilience of an individual and may be inversely associated with the occurrence of (immune mediated) chronic illnesses later in life reflected by survival increase. More traditionally, studies have evaluated immune responses to vaccines to predict protection against the tested agent or to speculate on the current functioning of the immune status. This is the first study relating this immune response following influenza vaccination in a relatively large study population to long-term survival. Gaining more information on the relation between immune system, its functioning and long-term effects is relevant since this might contribute to finding ways how to influence immune response and thus improve health benefits on the longer term. Moreover, discriminating between age groups might be particularly interesting given our previous findings of a potential effect of influenza vaccination on survival in those aged 60-64 years (chapter 4), but also considering the theory of immune senescence. Younger elderly could therefore be more responsive to vaccination, thus potentially, a relation between antibody response and survival might be better detectable.

In this study we could not demonstrate a relation between a higher antibody response following quadrivalent influenza vaccination and lower mortality in the elderly after 25 years of follow-up: the adjusted hazard ratio for mean antibody response following vaccination was $0.99(95 \% \mathrm{Cl}$ 0.88-1.10). Subgroup analysis by age showed an relation between antibody response and mortality in the youngest elderly (aged 60-64 years); adjusted hazard ratio 1.28 (95\% Cl 1.04-1.57). 
In our study, when compared to the youngest elderly aged 60-64, we did see relatively lower mean antibody responses following vaccination in those aged 65-69 and those aged 70+. Although these differences were statistically significant, such small differences in mean antibody response are not likely to be of any clinical relevance. Based on our findings, start of the decline in antibody response is likely to occur early in the aging process. This is largely in line with the previous findings of Thijs et al. showing that both elderly aged $70+$ and those aged 60 69 reached seroprotective titers for the most prominent strain (A/Beijing/353/89 [H3N2]), 30 leaving them to conclude that the decline of influenza vaccine efficacy with age is - at most modest, but differs by strains.

We found an inverse relation between antibody response and survival in the youngest elderly. This might be explained partially by the positive relation between antibody response after vaccination and the functioning of specific type of natural killer (NK) cells considered to harbor memory-like functions. ${ }^{31}$ These NK cells are a potential marker for immune senescence. During the process of aging, these NK cells (among other T-cells) could become more active and contribute to the activation of macrophages present in atherosclerotic plaques, eventually leading to plaque destabilisation and thus (cardiovascular) mortality. ${ }^{32}$ For this reason, the high titer responses we found in those aged 60-64 years might (indirectly) predict mortality. However, the study that describes the association between antibody response following vaccination and functioning of NK cells was not performed in an elderly population. Also, the proposed explanation does not completely clarify our findings, since in the older age groups, higher antibody responses following vaccination were not related to mortality. An alternative explanation could be found by stratifying the antibody response in those aged 60-64 years by smoking status. In a previous study based on the same data used in our new study, Cruijff et al. showed that the rise in antibody titre after vaccination was higher in smokers for certain strains. ${ }^{33}$ In our study, the relation between high antibody response and mortality is only seen in those participants that had smoked or were still smoking by time of inclusion in the 1991 trial. Thus, rest confounding related to smoking status might explain for the inverse relation between antibody response and mortality. Potentially also other confounders indicating an unhealthy lifestyle that are related to smoking status may contribute to this relation in patients with a history of smoking.

In literature, many different strains and antigens have been included to evaluate the process of immune senescence, making it hard to express immune senescence by one value of antibody response. Newer studies showed that it is rather the quantity of the immune response (expressed by the number of responding plasmablasts and hence plasmablast-derived polyclonal antibodies) that is reduced in elderly individuals, ${ }^{34}$ whereas the avidity and affinity of these antibodies (determining the quality of antibodies) is similar among age groups. ${ }^{34,35}$ Antibody titer is known to be a function of both quantity and quality of antibodies, thus revealing the overall reactivity of an antibody sample. ${ }^{34}$ Therefore we still do not exclude that response to influenza vaccination could be a reliable measure for reflecting the functioning of the humoral immune system more in general. 
Diagnosing influenza using point-of-care (POC) tests

In chapters 2 to 4 we discussed the role of influenza vaccination in reducing mortality in the elderly. Besides prevention by means of vaccination, also effective use of (new) diagnostic tools might contribute to reduction of the burden of disease caused by influenza. Therefore, in chapter $\mathbf{6}$ we evaluated the added value of point-of-care tests aiming to detect influenza. ${ }^{36}$ During an influenza-epidemic, workload of healthcare workers in both family practice as hospitals significantly increases. Also admission numbers are higher and length of in hospital stay increases. This might cause capacity problems and occlude the healthcare chain. Given the relatively low vaccine uptake in elderly that has shown decreasing trends over the past decade, the healthcare consumption might even further increase. For this reason, it is important to (re)consider the indications for referral or admission to the hospital. Therefore, in our narrative review as described in chapter 6, we evaluated the analytic accuracy of influenza POC tests, along with the added value for clinically relevant outcome measures; such as use of antibiotics and antiviral drugs, time spend on emergency department and length of hospital stay.

We concluded that POC tests that utilise antigen detection are user-friendly, but do have analytical accuracy limitations. POC tests based on PCR-technique are accurate, but rely on direct support from a medical laboratory. Therefore, these tests can only be deployed in hospitals. There is limited direct evidence for a clinical effectiveness of the influenza POC tests in the emergency department and there is a lack of proof concerning the clinical effectiveness of the influenza POC tests in a GP-practice setting. Influenza POC tests can reduce patients' length-of-stay in the ED and improve patient flow, provided there is capacity to accommodate this. Based on current available literature, it is warranted to deploy the PCR-based influenza POC tests in an ED setting. However, presently the implementation of the influenza POC tests in GP-practices cannot be justified.

\section{Methodology of studies}

Ethical analysis

An important part of the conclusions drawn in this thesis originate from our follow-up study based on an RCT. Our ethical analysis presented in chapter 2 , stressed the urge for this followup study. This ethical analysis followed a certain methodology which should be elaborated in the general discussion. We followed a systematic approach, in the first place by summarizing the findings and drawbacks of the existing evidence on various levels; i.e. observational studies, RCTs and (systematic) reviews. Besides these actual studies, also the interpretation of these data by various stakeholders is important. Therefore we discriminated these viewpoints on different levels: those writing the guidelines, i.e. the scientific community (for instance WHO and $\mathrm{CDC}$ ), those recommending and administrating influenza vaccination (i.e. the physicians) and those receiving influenza vaccination (i.e. the (elderly) patients). Consequently, we called in the expertise of an experienced bio-ethicist and applied various fundamental ethical principles to our 
research question 'Is an RCT of influenza vaccination on mortality justifiable?', such as: informed and voluntary participation under the condition of scientific justification for conducting the intended research, equipoise and proportionality of a trial (relating the importance of research to the potential disadvantages of the study participants).

Also, methodological barriers were discussed, since besides ethical feasibility, also the scientific yield and practical feasibility should be well considered. Direct evidence from trials evaluating the positive effect of influenza vaccination on short-term mortality in the elderly is lacking. Cohort studies often report reductions of $50 \%$ in the total risk of death in winter, whereas in fact only 5 to $10 \%$ of all winter deaths is attributed to influenza. ${ }^{22,37}$ The problem is that a postulated efficacy of influenza vaccination of 50\% in the elderly, means that total mortality can only be reduced by up to $2.5-5 \%$ by vaccination. Moreover, some studies found protective effects of influenza vaccination against mortality pre-epidemically ${ }^{38}$ or even in absence of influenza. ${ }^{39}$ In observational studies, the risk of bias and confounding is increased because of differences in characteristics between vaccinated and unvaccinated groups. Because the vaccination policy mainly targets persons at increased risk, one might expect an excess of unknown comorbidity in the group of vaccinees. This makes it hard to control observational studies for confounders. In practice however, the reverse is the case, known as 'healthy user' effect: in particular frail elderly would not receive vaccination, thus contributing to higher mortality rates in the group of unvaccinated elderly and therefore, increasing vaccine effectiveness in terms of mortality reduction. ${ }^{40}$ The importance of confounders in observational influenza vaccine studies is stressed by a systematic review on studies evaluating the effect of influenza vaccines which showed that when selection bias is better avoided, chances of a favorable conclusion about the vaccine effect are significantly lower. ${ }^{41}$ Various attempts have been made to correct for confounders; for instance by modeling the effect of a hypothetical unmeasured confounder that would have caused overestimation of vaccine effectiveness, ${ }^{42}$ adjusting for differences in mortality between vaccinated and unvaccinated individuals following the influenza season ${ }^{43}$ or more complete adjustment for functional and socioeconomic status. ${ }^{39}$ Despite this, the findings of these studies are not conclusive. ${ }^{40}$

Ideally, the first trials evaluating the effects of influenza vaccination on morbidity as an outcome, would also have taken hospitalizations or mortality into account. However, by that time, trials were primarily aimed at preventing influenza as such. A trial evaluating an infrequent endpoint as hospitalization or mortality requires 100,000 up to even over 500,000 elderly to be included in order to prove for an effect of influenza vaccination on mortality related to influenza. ${ }^{2,3}$ Other methodological challenges also lie in the study period during which the efficacy of the vaccine is to be assessed. Since the incidence of influenza varies annually and vaccine mismatches occur regularly, such trials would ideally cover more than one season in order to increase its external validity, proposing even more difficulties in conducting them. Finally, an RCT would logically only include vaccine refusers (given the fact that influenza vaccination is considered competent-care in most western countries). It goes without saying 
that including vaccine refusers in a vaccination trial might be problematic from practical point of view. Besides, as previously stated, a head-to-head trial between newer influenza vaccines and the present vaccine may seem ethically feasible, but would require such high numbers of participants to prove for an effect on mortality in favor of the newer vaccine, that such a trial would become methodologically challengeable.

\section{Follow-up studies in the elderly}

Given the limitations addressed in the previous paragraph, it is unlikely that an answer to the question whether influenza vaccination reduces mortality in the elderly will soon be supported by direct RCT evidence. We hypothesized that by preventing (accumulation of) influenza-related complications and residual protection that lasts more than just one influenza season, influenza vaccination could reduce mortality on the longer term. We found ourselves in the unique position to conduct a 25 -year followup on mortality of an RCT on the efficacy of influenza vaccination in the elderly in the 1991/1992 winter season. By evaluating this RCT, any survival effect seen in the vaccinated group might be attributed to the vaccine given the randomized nature of this study, thus providing for the only direct evidence available on long-term effects of influenza vaccination on mortality in community-dwelling elderly. Also, this follow-up study would provide us exclusive data in order to study the relationship between the humoral immune response following vaccination and mortality on the long term. In case we would have found an effect of single vaccination on mortality in this follow-up study of a trial, one might question whether this is truly due to a single influenza vaccination administered in 1991/1992. Various methodological aspects are important to consider when interpreting the findings of such a follow-up study. For instance, random allocation to vaccine or placebo group is a relevant fact which is at the basis of any potential differences in survival between vaccine and placebo group. Because participants had not been de-blinded at end of the trial, both randomized groups were likely to have been (re)vaccinated in equal proportions. Also, by the time of conducting the 1991 trial, uptake of influenza vaccinations was low and vaccination recommendations for those aged $>65$ were only introduced in 1996. Thus, for a substantial part of the participants, the 1991 trial vaccination was likely to have been the first and for a long time only influenza vaccination received. In case of any effects found in such a follow-up study, this should be related to the difference between the studied groups at baseline; in this case influenza vaccination in 1991/1992.

Also, in follow-up studies like these, the power regarding an outcome measure that was not defined in the original trial as primary endpoint should be carefully considered. Numbers in such a study are likely to be too small to relate vaccination directly to influenza deaths in the influenza season occurring that year. A high rate of complete follow-up - like in our study - can actually result in high numbers of person years of follow-up and events, thus increasing the study power for mortality as an outcome.

Finally, also the quality of the primary study which is being used is - obviously - vital for a cohort study based on that RCT. For instance, the RCT should be well documented; i.e. besides age and gender, also other covariates like current diseases, smoking status or socio-economic 
status are important to consider. Besides that, it would be helpful if such a study would be made suitable for follow-up. For example, one could check already at time of including participants, which demographic details are required to extract mortality data from the national institution that keeps person records later on.

Survey among GPS

In chapter 3 we conducted a survey among 500 GPs in the Netherlands. Our methodological approach allowed us to compare survey responses before and during the influenza season. Also - by adopting some questions of a previous study ${ }^{10}$ - we could compare GP vaccination rates over time. Finally, this study evaluates currently debated topics regarding influenza vaccination (i.e. mandatory HCW influenza vaccination and desirability of an influenza vaccination trial on mortality) that have not been evaluated in such large populations of GPs before. Up to our knowledge, no studies quantified the effect of seasonality on attitudes of GPs on influenza vaccination before. A qualitative Australian study did suggest that the severity of the previous influenza season could affect the attitude of stakeholders towards mandatory influenza vaccination in $\mathrm{HCW}$ one year later. ${ }^{44}$ Although we examined this effect within the same season, we could not demonstrate a significant effect of the epidemic occurrence of influenza on the GP's attitude towards mandatory influenza vaccination in HCW. It should be noted that the 2018/2019 influenza season in the Netherlands was relatively mild, potentially limiting the near significant effect. Our finding that GPs' attitudes towards mandatory influenza vaccination in HCW does not significantly change during the influenza season should be interpreted with care, as explained previously. In case stakeholders or HCWs are involved in deciding on such a mandate, policymakers should be aware that, especially during a severe influenza epidemic, a potential seasonal effect on this opinion, cannot be ruled out.

\section{CONCLUSION}

In this thesis we examined how the impact of influenza in primary care can be reduced following practical and theoretical perspectives. It contributes to the body of evidence by providing an ethical and methodological framework for new influenza vaccination trials, demonstrating the attitudes and beliefs of GPs regarding vaccination, evaluating long-term effects of vaccination on mortality, exploring the relation between immune response to vaccination and long-term mortality, and underlining the relevance of influenza point-of-care tests in different settings. 


\section{REFERENCES}

1. Govaert TM, Thijs CT, Masurel N, Sprenger MJ, Dinant GJ, Knottnerus JA. The efficacy of influenza vaccination in elderly individuals. A randomized double-blind placebo-controlled trial. Jama. 1994;272:1661-5.

2. Verhees RAF, Dondorp W, Thijs C, Dinant GJ, Knottnerus JA. Influenza vaccination in the elderly: Is a trial on mortality ethically acceptable? Vaccine. 2018;36:2991-7.

3. Ekkelenkamp MB, van Werkhoven CH, Bruijning-Verhagen PCJ, Bonten MJM. [A trial to study the effect of influenza vaccination in the elderly: ethical, feasible and badly needed]. Ned Tijdschr Geneeskd. 2018;162.

4. van Beek J, Veenhoven RH, Bruin JP, et al. Influenza-like IIIness Incidence Is Not Reduced by Influenza Vaccination in a Cohort of Older Adults, Despite Effectively Reducing Laboratory-Confirmed Influenza Virus Infections. J Infect Dis. 2017;216:415-24.

5. Corrales-Medina VF, Musher DM, Wells GA, Chirinos JA, Chen L, Fine MJ. Cardiac complications in patients with community-acquired pneumonia: incidence, timing, risk factors, and association with short-term mortality. Circulation. 2012;125:773-81.

6. Molinari NA, Ortega-Sanchez IR, Messonnier ML, et al. The annual impact of seasonal influenza in the US: measuring disease burden and costs. Vaccine. 2007;25:5086-96.

7. Knottnerus JA. Influenza vaccination in the elderly: current evidence and uncertainties. J Clin Epidemiol. 2009;62:675-6.

8. Chalmers I, Glasziou P. Avoidable waste in the production and reporting of research evidence. Lancet. 2009;374:86-9.

9. Verhees RAF, Snellings R, Dinant GJ, Knottnerus JA. Influenza vaccination among Dutch general practitioners and their attitude toward influenza vaccination in the elderly. Hum Vaccin Immunother. 2020;16:2709-18.

10. Opstelten W, van Essen GA, Ballieux MJ, Goudswaard AN. Influenza immunization of Dutch general practitioners: vaccination rate and attitudes towards vaccination. Vaccine. 2008;26:5918-21.

11. Opstelten W, van Essen GA, Heijnen ML, Ballieux MJ, Goudswaard AN. High vaccination rates for seasonal and pandemic $(\mathrm{A} / \mathrm{H} 1 \mathrm{~N} 1)$ influenza among healthcare workers in Dutch general practice. Vaccine. 2010;28:6164-8.

12. Health council of the Netherlands. Influenza vaccination: revision of the indication [cited January 2020]. Available from: https://www.healthcouncil.nl/documents/advisory-reports/2007/03/08/ influenza-vaccination-revision-of-the-indication.

13. Collange F, Verger P, Launay O, Pulcini C. Knowledge, attitudes, beliefs and behaviors of general practitioners/family physicians toward their own vaccination: A systematic review. Hum Vaccin Immunother. 2016;12:1282-92.

14. Lorenc T, Marshall D, Wright K, Sutcliffe K, Sowden A. Seasonal influenza vaccination of healthcare workers: systematic review of qualitative evidence. BMC Health Serv Res. 2017;17:732

15. deSante JE, Caplan A, Shofer F, Behrman AJ. Physician attitudes towards influenza immunization and vaccine mandates. Vaccine. 2010;28:2517-21.

16. Medisch Contact. Column about influenza vaccination in HCWs [cited July 2021]. Available from: https://www.medischcontact.nl/opinie/blogs-columns/column/de-griepprik-bij-artsen-enverzorgenden.htm.

17. Armstrong BG, Mangtani P, Fletcher A, et al. Effect of influenza vaccination on excess deaths occurring during periods of high circulation of influenza: cohort study in elderly people. BMJ. 2004;329:660. 
18. Verhees RAF, Thijs C, Ambergen T, Dinant GJ, Knottnerus JA. Influenza vaccination in the elderly: 25 years follow-up of a randomized controlled trial. No impact on long-term mortality. PLoS One. 2019;14:e0216983.

19. Gavazzi G, Krause KH. Ageing and infection. Lancet Infect Dis. 2002;2:659-66.

20. Haralambieva IH, Painter SD, Kennedy RB, et al. The impact of immunosenescence on humoral immune response variation after influenza A/H1N1 vaccination in older subjects. PLoS One. 2015;10:e0122282.

21. Thomas RE. Are influenza-associated morbidity and mortality estimates for those $\geq 65$ in statistical databases accurate, and an appropriate test of influenza vaccine effectiveness? Vaccine. 2014;32:6884901.

22. Jansen AG, Sanders EA, Hoes AW, van Loon AM, Hak E. Influenza- and respiratory syncytial virusassociated mortality and hospitalisations. Eur Respir J. 2007;30:1158-66.

23. Luytjes W, Enouf $\mathrm{V}$, Schipper M, et al. HI responses induced by seasonal influenza vaccination are associated with clinical protection and with seroprotection against non-homologous strains. Vaccine. 2012;30:5262-9.

24. Dou Y, Fu B, Sun R, et al. Influenza vaccine induces intracellular immune memory of human NK cells. PLoS One. 2015;10:e0121258.

25. McLean HQ, Thompson MG, Sundaram ME, et al. Impact of repeated vaccination on vaccine effectiveness against influenza A(H3N2) and B during 8 seasons. Clin Infect Dis. 2014;59:1375-85.

26. Goodwin K, Viboud C, Simonsen L. Antibody response to influenza vaccination in the elderly: a quantitative review. Vaccine. 2006;24:1159-69.

27. Praditsuwan R, Assantachai P, Wasi C, Puthavatana P, Kositanont U. The efficacy and effectiveness of influenza vaccination among Thai elderly persons living in the community. J Med Assoc Thai. 2005;88:256-64.

28. Voordouw BC, van der Linden PD, Simonian S, van der Lei J, Sturkenboom MC, Stricker BH. Influenza vaccination in community-dwelling elderly: impact on mortality and influenza-associated morbidity. Arch Intern Med. 2003;163:1089-94.

29. Clar C, Oseni Z, Flowers N, Keshtkar-Jahromi M, Rees K. Influenza vaccines for preventing cardiovascular disease. Cochrane Database Syst Rev. 2015:Cd005050.

30. Thijs C, Beyer WE, Govaert PM, Sprenger MJ, Dinant GJ, Knottnerus A. Mortality benefits of influenza vaccination in elderly people. Lancet Infect Dis. 2008;8:460-1; author reply 63-5.

31. Riese P, Trittel S, Pathirana RD, Klawonn F, Cox RJ, Guzmán CA. Responsiveness to Influenza Vaccination Correlates with NKG2C-Expression on NK Cells. Vaccines (Basel). 2020;8.

32. JW. T. Tervaert JW. Immunologie: het hart van de zaak. Maastricht: Maastricht University, 2001.

33. Cruijff M, Thijs C, Govaert T, Aretz K, Dinant GJ, Knottnerus A. The effect of smoking on influenza, influenza vaccination efficacy and on the antibody response to influenza vaccination. Vaccine. 1999;17:426-32.

34. Sasaki S, Sullivan M, Narvaez CF, et al. Limited efficacy of inactivated influenza vaccine in elderly individuals is associated with decreased production of vaccine-specific antibodies. J Clin Invest. 2011;121:3109-19.

35. Blomberg BB, Frasca D. Quantity, not quality, of antibody response decreased in the elderly. J Clin Invest. 2011;121:2981-3.

36. Verhees RAF, Lutgens SPM, Kusters R, Dinant GJ, Cals JWL. [Influenza point-of-care test in the GP practice and Emergency Department; analytical accuracy and added value]. Ned Tijdschr Geneeskd. 2019;163. 
37. Simonsen L, Taylor RJ, Viboud C, Miller MA, Jackson LA. Mortality benefits of influenza vaccination in elderly people: an ongoing controversy. Lancet Infect Dis. 2007;7:658-66.

38. Jackson LA, Jackson ML, Nelson JC, Neuzil KM, Weiss NS. Evidence of bias in estimates of influenza vaccine effectiveness in seniors. Int J Epidemiol. 2006;35:337-44.

39. Eurich DT, Marrie TJ, Johnstone J, Majumdar SR. Mortality reduction with influenza vaccine in patients with pneumonia outside "flu" season: pleiotropic benefits or residual confounding? Am J Respir Crit Care Med. 2008;178:527-33.

40. de Jong JC, Hak E, Osterhaus AD. [Influenza vaccination in the elderly makes sense. Many confounding factors in observational research]. Ned Tijdschr Geneeskd. 2010;154:A1171.

41. Jefferson T, Di Pietrantonj C, Debalini MG, Rivetti A, Demicheli V. Relation of study quality, concordance, take home message, funding, and impact in studies of influenza vaccines: systematic review. BMJ. 2009;338:b354.

42. Nichol KL, Nordin JD, Nelson DB, Mullooly JP, Hak E. Effectiveness of influenza vaccine in the community-dwelling elderly. N Engl J Med. 2007;357:1373-81.

43. Groenwold RH, Hoes AW, Hak E. Impact of influenza vaccination on mortality risk among the elderly. Eur Respir J. 2009;34:56-62.

44. Moran A, Agaliotis M, Seale H. The views of key stakeholders around mandatory influenza vaccination of hospital and aged care staff: Examining the current climate in Australia. Vaccine. 2019;37:705-10. 


Addendum

Impact paragraph 



\section{IMPACT PARAGRAPH}

In the previous chapter I focused on our main findings and placed these findings in a broader context. This concluding paragraph will give a reflection on the practical, societal and scientific impact of the results of the research described in this thesis.

\section{Relevance for patients and society}

During an influenza epidemic, up to $10 \%$ of the people is affected by influenza. In addition to its unpleasant symptoms, influenza also gives an increased risk of serious complications or even death. Many patients are at risk for complications due to influenza, especially the elderly. Besides morbidity and mortality caused by influenza, also loss of productivity and increased hospital workload contribute to its societal impact. Whereas we know that influenza vaccination can prevent morbidity, an effect on reducing mortality by vaccination has never been proven with direct RCTbased evidence. Since vaccine hesitancy in elderly is high (approximately $45 \%$ of the elderly is not receiving influenza vaccination), ${ }^{1}$ better understanding of long-term effects of vaccination might help patients deciding on receiving influenza vaccination or not. We have contributed to the body of evidence of long-term effects of influenza vaccination on mortality in the elderly, trying to support this with direct evidence. Although we could not prove for an effect, we shared findings of possible survival benefit of up to 20 months in the younger elderly aged 60 to 65 years. These findings might encourage patients (especially the younger elderly) to receive influenza vaccination.

Impact on the healthcare system

Due to the high incidence, the risk of serious complications and the increasing age of population, the burden of disease caused by influenza is high. ${ }^{2}$ As a result, the workload of doctors and nurses in primary and secondary care increases during the flu seasons, as well as bed occupancy and the length of hospitalization. ${ }^{3,4}$ Suddenly increased elderly care needs but also absenteeism among caregivers and healthcare workers (HCWs) due to influenza can disrupt the healthcare system. ${ }^{5}$ Besides vaccinating high-risk patients against influenza, also HCW vaccination is assumed to reduce its impact by preventing absenteeism and transmission to elderly and their caregivers. ${ }^{6}$ However, many HCWs choose to stay unvaccinated. Determinants of vaccine refusal in HCWs have been studied extensively but mostly in hospitals or elderly homes, whereas in many high-income countries like the Netherlands, general practitioners (GPs) are key actors in implementing influenza vaccination policy. By studying the attitudes and beliefs of Dutch GPs regarding influenza vaccination in general but also more specifically towards influenza vaccination in HCWs and elderly we aimed to gain more insight in how coverage rates in different targeted groups could be increased. We found a self-reported coverage rate of influenza vaccination of $71.9 \%$ in GPs. In the absence of surveillance systems monitoring influenza vaccination coverage rates in primary care on any (national or European) level, our up-to-date information on GP coverage rates and their attitudes towards influenza vaccination is relevant for the current healthcare system. 
To cope with the higher demand for care previously mentioned, it is important to critically review the referral and admission criteria for patients with influenza symptoms. Therefore, in addition to prevention through vaccination, early diagnosis of influenza could also help limiting the impact of influenza. We have described the accuracy of rapid influenza diagnostic tests and their effect on clinically relevant outcome measures, such as use of antibiotics and antiviral drugs, length of stay at the emergency department (ED) and length of hospitalization. We concluded that the current literature warrants the use of point-of-care (POC) PCR-based influenza tests at emergency departments as long as rapid diagnostics are not used at the expense of careful diagnostics. Implementation of influenza POC tests in general practice is, for the time being, a bridge too far. We have set out aspects for successful implementation of a diagnostic test showing that not only its analytical accuracy and clinical efficacy are important, but also other factors such as the practicality and cost-effectiveness of the test, the interpretation of the test results by the user, and the level of stress experienced by patients undergoing the test are important to consider. We have also made recommendations for how and in which conditions POC tests for influenza could be used in different settings: the general practice and the emergency department. Especially given the current advance of POC tests, GPs should now be able to determine whether these tests would have an additional value for their practice. In the emergency departments, we have already seen that in the winter of 2019/2020 POC PCR-based influenza tests were used more frequently than in previous years.

\section{Impact on research}

The effectiveness of influenza vaccination in the elderly has long been a topic of debate, fuelled by the absence of direct evidence from randomized trials on its effect on mortality and the methodological limitations of observational studies pointing this direction. In our survey study among Dutch GPS, we have seen that $60.5 \%$ of the GPs desires a placebo-controlled trial on influenza vaccination in the elderly evaluating mortality as an endpoint and that $86.1 \%$ would have no objections if their elderly patients would participate in such a trial, exposing them to the harmful risks of influenza. This (uninformed) opinion has no direct implications for the justification and feasibility of such a trial. However, it should be seen as an important sign for the scientific community to clearly communicate on the state of evidence on the effects of influenza vaccination on morbidity and mortality in the elderly and the substantial ethical, methodological and practical barriers to be addressed before a placebo-controlled trial with severe complications and mortality as outcomes would be justified. Also, if new research in the field is conducted, this study provides new insights in knowledge gaps or outstanding questions that could be addressed. For instance, the observed uncertainty among GPs in our study on the need for influenza vaccination in 'healthy' elderly.

In chapter 2, we have come with a framework indicating these ethical and methodological challenges of a hypothetical placebo-controlled influenza vaccination trial in the elderly with mortality as an endpoint. This framework could be used by researches considering to conduct new influenza vaccination trials in the elderly. Moreover, it could be used for other vaccination studies that consider to evaluate long-term mortality as an endpoint. 
In chapter 4, we have shown the relevant findings of a beneficial effect of single influenza vaccination on survival in the subgroup aged 60-64 years, which merits additional research on the effect of vaccination on long-term mortality in the elderly. Therefore, we recommend researchers intending to conduct studies on the efficacy of influenza vaccines, to accustom these studies for longer follow-up. Moreover, these results could encourage researchers to compare the impact of influenza vaccination on long-term survival in younger and older vaccinated elderly and to study the long-term effect of influenza vaccination on immune memory.

Finally, in our explorative study in chapter 5 we have shown that there is no relation between higher levels of antibody response following influenza vaccination and lower long-term mortality in the elderly. Whereas we do know that the level of titer increase after vaccination correlates with clinical protection against the virus, we have not demonstrated that this type of immune response can be interpreted as a wider indicative marker for immune responsiveness translating into higher chances for survival. Although replication of these study findings is needed, we think research should now also focus on other markers that might be indicative for immune responsiveness in general.

\section{Impact on policy making}

In the Netherlands, influenza vaccination in the elderly (aged 65 and older) has been recommended by the guidelines for GPs ('NHG-standaarden') since 1996. In 2007, the Health Council of the Netherlands ('Gezondheidsraad') proposed to expand the risk group and include everyone from the age of 60 instead of 65 . Up to present time these recommendations last for this risk group. Our new studies do not change the scientific ground upon which these recommendations are based.

Recently, a report carried out on behalf of the ministry of Health, Welfare and Sport showed that there is currently no legal basis for obligation of influenza vaccination that the employer can impose, and that there are legal objections to the introduction of an obligation. ${ }^{7}$ Moreover, there is modest support for it from organisations of employers and employees, partly because they expect an obligation to arouse resistance from healthcare providers. In chapter 3 , we have shown that most GPs are vaccinated and recommend influenza vaccination to their practice personnel. The high vaccination coverage in GPs as such, the hesitancy of GPs to mandate influenza vaccination in $\mathrm{HCW}$ s and the fact that non-institutionalized elderly are easily exposed to many potential carriers of influenza other than $\mathrm{HCW}$ s should all be taken in to account when policymakers decide on mandatory influenza vaccination for HCWs in general and - more explicitly - in primary care.

Elaboration on the new corona virus (SARS-CoV-2): lessons learned from this thesis During the writing of the last chapters of this thesis, the world was confronted with a new pandemic. This time however, it was not a new strain of influenza virus, but a corona virus that circulated the globe, causing substantial morbidity and mortality, occlusion of the healthcare 
chain and a disruption of our social and economic society. It seems evident to devote a paragraph in this thesis to the lessons learned from this thesis that can be applied to SARS-CoV-2, current SARS-CoV-2-related research and (testing) policy.

In the context of limited pre-existing knowledge of SARS-CoV-2, this pandemic demonstrated the importance of a close collaboration between the scientific community and policy makers in order to reduce its societal impact. In addition, researchers were confronted with a number of specific methodological and practical challenges. Whereas normally animal studies precede human trials to test a vaccine for safety and effectiveness, now in order to speed up vaccine development, some SARS-CoV-2 vaccines were being tested in both animal and phase I trials simultaneously. By that time, it was still unclear whether vaccinated people would become less ill due to the virus and whether those who did get infected would develop a more severe form of the disease than infected unvaccinated people (a phenomenon called 'disease enhancement'). It was expected that the risk of enhancement was low, but the risk of not getting vaccines advanced quickly, was fairly high if the prior risk would hold back development of vaccines too strongly. ${ }^{8}$ Also, by the time an important producer of vaccines submitted their studies for FDA approval, only vaccine efficacy on getting COVID-19 (the disease associated with SARS-CoV-2 infection) as such had been evaluated as primary objective, whereas the studies previously performed were not powered on mortality or hospitalization. This is comparable to the policy decision process that was applied to influenza vaccination, in which evidence for prevention of the disease itself was considered sufficient in order to justify large scale vaccination, while direct evidence for reducing mortality was still lacking. In the specific situation in which an intervention becomes standard care before its efficacy has been proven on all relevant outcome measures, making studies suitable for follow-up may provide additional evidence on mortality or rare side affects. One might think that a similar ethical and methodological framework as proposed in chapter 2 would also apply to new SARS-CoV-2 vaccination trials that researchers might want to conduct in the future. However, for influenza vaccination this is more complicated since many SARSCoV-2 trials have been carried out in one season and could be easily continued until efficacy on the more rare outcomes such as mortality was demonstrated. In the case of influenza vaccination, new trials should be started from a situation of non-equipoise, and moreover, lethality of influenza is lower than lethality of COVID-19 so that a much larger trial would be needed. This challenge is ethically, methodologically and practically much more complex (see also chapter 2).

Besides registration of the vaccination status in research setting, the impact of the SARS-CoV-2 pandemic has demonstrated the need of adequate vaccination registration on the individual level. A pre-existing system for influenza vaccination registration might have helped easing the registration of SARS-CoV-2 vaccines. Vice versa, the current pandemic might provide for a strong argument for a more detailed influenza registration on the individual level in order to be prepared for emerging influenza viruses.

The SARS-CoV-2 pandemic also created large scale public awareness on the pro's and con's of vaccination. Recently published results show that over a quarter of people living in Europe indicate a hesitancy towards the SARS-CoV-2 vaccine. ${ }^{9}$ Concern about safety is the biggest reason 
for vaccine hesitancy (62\%) but 44\% of those indicating hesitancy believe the health risk of SARSCoV-2 is exaggerated. From the very moment on when SARS-CoV-2 vaccines became available to the public, family practices were flooded by patients who wanted to speak to their GP to inform about the vaccine: 'Should I take it?', 'Is it safe?' This stresses the importance of the family physician in influencing the willingness of people to receive vaccination. We should not forget that for this reason it remains important to stay updated on the attitudes and beliefs of GPs about vaccination. Only when GPs are well informed, they can sent out a correct and clear message to the public.

In light of the SARS-CoV-2 pandemic, it is relevant to elaborate on the definition of 'test accuracy' used in chapter 6 . The PCR-test is considered the gold standard for diagnosing a SARSCoV-2 infection. The cycle threshold (CT) value of a PCR-test refers to the number of cycles needed to amplify viral RNA to reach a detectable level. Although a test with the slightest amount of detected RNA can be labelled positive, recent studies have shown that in samples with CT values $\geq 32$, virus replication no longer occurs, so that the person in question can be considered 'noninfectious.'10 Discriminating potentially infectious patients from others is important from public health perspective, focusing for instance on containing the virus / reduce spreading of the virus. In chapter 6 we did not make a clear discrimination between the different aims of a test: detecting clinically ill patients vs. infectious patients. This is because in specific settings like hospitals and GP-practices, also people with a low viral loads need to be detected since even low amounts of viral RNA could still be dangerous in or to vulnerable and immunocompromised patients. Finally, there are also parallels between our study on influenza POC testing and testing for SARS-CoV-2. Given the urge for rapid testing in emergency departments, high test accuracy and proven effects on important outcome measures, the first influenza POC PCR-tests were already introduced in non-research setting in the winter of 2019/2020. Multiple antigen detection tests for SARS-CoV-2 have also become available in the meantime, but as is also true for the influenza antigen detection tests, these tests have a relatively low sensitivity, increasing the risk on false negative results. ${ }^{10}$ In the development of better POC tests, the same criteria for a good test as we mentioned for influenza POC tests should apply. Based on our study presented in chapter 6, GPs should be able to determine whether these tests would have added value for their practice.

\section{FINAL CONCLUSION}

The impact caused by influenza on the individual and society is significant and can partly be prevented or decreased by influenza vaccination and point-of-care testing in specific settings such as emergency departments. The general practitioner is a key actor in implementing influenza vaccination and the GPs' attitudes and beliefs regarding vaccination are important as these can serve as promotors of the vaccination policy. Finally, this thesis has the goal to stress the potency of long-term follow-up of intervention studies and the importance of considering ethical and methodological aspects of research when it comes to developing new influenza vaccination trials. 


\section{REFERENCES}

1. Monitor vaccination coverage rate in the Netherlands by Nivel [cited May 2021]. Available from: https:// www.nivel.nl/nl/publicatie/monitor-vaccinatiegraad-nationaal-programma-grieppreventie-2019.

2. van Lier A, McDonald SA, Bouwknegt M, et al. Disease Burden of 32 Infectious Diseases in the Netherlands, 2007-2011. PLoS One. 2016;11:e0153106.

3. Sauro A, Barone F, Blasio G, Russo L, Santillo L. Do influenza and acute respiratory infective diseases weigh heavily on general practitioners' daily practice? Eur J Gen Pract. 2006;12:34-6.

4. Beysard N, Yersin B, Meylan P, Hugli O, Carron PN. Impact of the 2014-2015 influenza season on the activity of an academic emergency department. Intern Emerg Med. 2018;13:251-56.

5. European Commission. Importance of HCW vaccination [cited October 2019]. Available from: https:// ec.europa.eu/health/vaccination/influenza_en.

6. Maltezou HC, Poland GA. Vaccination policies for healthcare workers in Europe. Vaccine. 2014;32:4876-80.

7. Report evaluating mandatory HCW vaccination in the Netherlands [cited July 2021]. Available from: https://www.rijksoverheid.nl/documenten/rapporten/2019/12/06/verplichting-griepvaccinatie-bijzorgwerkers-wenselijk-en-mogelijk.

8. Nature magazine [cited July 2020]. Available from: https://www.nature.com/articles/d41586-02000798-8.

9. European Foundation for the Improvement of living and working conditions. Questionnaire on vaccine hesitancy [cited July 2021]. Available from: https://www.eurofound.europa.eu/publications/ report/2021/living-working-and-covid-19-update-april-2021-mental-health-and-trust-declineacross-eu-as-pandemic.

10. Smits F, Torensma B, Groenwold RH, et al. SARS-CoV-2 antigen test insufficiently accurate for screening in primary care. Huisarts Wet 2021;64. 


Addendum

Samenvatting 



\section{SAMENVATTING}

Jaarlijks krijgt tot 10\% van de algemene bevolking te maken met influenza of de gevolgen daarvan. Deze kunnen ernstig zijn en bijvoorbeeld tot ziekenhuisopname of zelfs sterfte leiden. Met name ouderen en mensen met chronische aandoeningen (bijvoorbeeld longof hartaandoeningen) lopen extra risico hierop. Influenzavaccinatie kan de kans op het krijgen van griep onder ouderen met ca. 50\% reduceren. Daarmee is vaccineren vooralsnog de belangrijkste strategie om de impact van influenza te beperken. Ook vaccinatie van gezondheidszorgpersoneel kan een belangrijke schakel hierin zijn. Echter, de vaccinatiegraad van zowel ouderen als gezondheidszorgpersoneel ligt altijd nog onder het streefnivo dat de Wereldgezondheidsorganisatie hiervoor aanhoudt. Indien onvoldoende aandacht uitgaat naar de preventie en bestrijding van influenza, kan een influenza-epidemie tot plotse toename van de zorgbehoefte leiden, waardoor capaciteitsproblemen in de zorgketen kunnen ontstaan. Daarom onderzoek ik in dit proefschrift hoe de impact van influenza vanuit verschillende invalshoeken gereduceerd kan worden. Er blijft veel discussie bestaan ten aanzien van de vraag of influenzavaccinatie ook echt ziekenhuisopnames en sterfte onder ouderen kan voorkomen. Om die reden heb ik in kaart gebracht welke ethische en methodologische barrières overwonnen zouden moeten worden, wil er nog ooit een placebo-gecontroleerde studie naar het effect van influenzavaccinatie op sterfte onder ouderen worden gedaan. Omdat ik concludeer dat dit zeer problematisch lijkt, heb ik gebruik gemaakt van een belangrijke influenzastudie die ruim 25 jaar geleden is uitgevoerd, en bestudeerd of uit deze studie bewijs voortkomt voor een langduriger, niet-seizoensgebonden effect van eenmalige influenzavaccinatie op sterfte onder ouderen. Ik heb het vaccinatiegedrag van huisartsen bestudeerd, omdat zij in Nederland een sleutelrol vervullen in het influenzavaccinatiebeleid. Ook onderzocht ik de meerwaarde van sneltests voor influenza in verschillende settings: de huisartsenpraktijk en de eerste hulp. Tot slot bestudeerde ik de relatie tussen de immuunreactie op een influenzavaccin en sterfte op de langere termijn.

In hoofdstuk 2 hebben we de belangrijke studies die zich richten op het effect van influenzavaccinatie op sterfte onder ouderen op een rij gezet. Vervolgens hebben we weergegeven welke hiaten er zijn in deze kennis. Er is immers geen direct bewijs voor een effect van influenzavaccinatie op sterfte onder ouderen, juist omdat studies die deze uitkomstmaat onderzoeken nooit met deze intentie zijn opgezet. De gerandomiseerde trials die zijn uitgevoerd hebben zich gericht op de uitkomstmaten ziekte door influenza of laboratorisch bewezen infectie met influenza. Dit mede vanuit de gedachte dat door influenza te voorkomen, ook sterfte door influenza voorkomen wordt. Daarnaast zou een trial gericht op sterfte zeer grote aantallen deelnemers vereisen. Er zijn wel studies uitgevoerd naar het effect op sterfte, maar deze studies zijn observationeel, veelal van minder goede kwaliteit en de resultaten van deze studies spreken elkaar tegen. Een direct effect kan middels deze studies ook niet worden aangetoond, omdat de gevaccineerde en ongevaccineerde groepen vaak niet goed te vergelijken zijn en hiervoor moeilijk 
volledig te corrigeren is. Het is begrijpelijk dat er een behoefte bestaat aan eenduidig bewijs van het effect van influenzavaccinatie op sterfte onder ouderen (zie ook de behoefte hieraan onder huisartsen in hoofdstuk 3). Echter, een belangrijke conditie in de ethische rechtvaardiging van een dergelijk gerandomiseerd onderzoek is het bestaan van voldoende onzekerheid ten aanzien van de onderzoeksvraag. Uitvoerige analyse van al het bewijs van influenzavaccinatie op sterfte onder ouderen, wijst in de richting van een beschermend effect. Daarnaast geldt ook een plausibilteitsprincipe dat stelt dat door influenza te voorkomen, je ook de complicaties door influenza zoals hospitalisatie en sterfte kunt voorkomen. Dit samen maakt het onthouden van een influenzavaccin aan kwetsbare proefpersonen in een nieuwe influenzatrial moeilijk te verdedigen, niet in de laatste plaats omdat influenzavaccinatie in Nederland deel uitmaakt van de standaardzorg. Je zou deze mensen dan de standaardzorg waarvan we denken dat deze ten goede van de oudere patiënt komt en complicaties door influenza voorkomt, moeten onthouden. Het belang van een trial weegt daarmee vermoedelijk niet op tegen de risico's waar je patiënten in een dergelijk onderzoek aan blootstelt. Naast de balans tussen risico's versus voordelen van een trial gericht op sterfte, geldt ook dat influenzavaccinatie zich al in meerdere studies op andere relevante (beleidsmatige) uitkomstmaten heeft bewezen, waaronder kosteneffectiviteit. Onderzoekers zouden kunnen overwegen een placebo-gecontroleerde studie uit te voeren waarin vaccinweigeraars worden geïncludeerd. Echter, vaccinweigeraars vormen geen representatieve groep waardoor de generaliseerbaarheid van zo'n studie naar de algemene populatie in het gedrang komt. Tevens zou in een trial met sterfte als uitkomstmaat een onrealistisch groot aantal deelnemers geïncludeerd moeten worden. Ten slotte stuit je op een praktische paradox; waarom zouden vaccinweigeraars mee willen doen aan een vaccinstudie? Mogelijkheden zouden gezocht kunnen worden in het opzetten van 'head-to-head' trials waarin het reguliere influenzavaccin met een nieuw influenzavaccin vergeleken wordt. Echter, omdat de verschillen tussen de onderzochte groepen hierin naar verwachting kleiner worden, wordt het nog lastiger om een verschil op een zeldzame uitkomst als sterfte aan te tonen, laat staan superioriteit van een nieuw vaccin aan te tonen. We concluderen derhalve dat er meerdere ethische en methodologische bezwaren zijn, die maken dat een placebo-gecontroleerde studie naar het effect van influenzavaccinatie onder ouderen op sterfte als uitkomstmaat moeilijk te verdedigen en te realiseren is.

De huisarts speelt een sleutelrol in het influenzavaccinatiebeleid in Nederland. Gezien deze achtergrond is hun houding t.a.v. influenzavaccinatie onder gezondheidszorgpersoneel en ouderen, maar ook influenza gerelateerd onderzoek relevant. In hoofdstuk $\mathbf{3}$ onderzochten we de houding van Nederlandse huisartsen ten aanzien van influenzavaccinatie onder ouderen en praktijkpersoneel, vóór en tijdens de influenza-epidemie van 2018/2019. Van de huisartsen gaf 72\% aan zich voor het influenzaseizoen 2018-2019 gevaccineerd te hebben. De belangrijkste redenen hiervoor waren het verkleinen van het risico zelf influenza te krijgen en het verkleinen van het risico patiënten te besmetten. De belangrijkste redenen om van vaccinatie af te zien 
waren het niet behoren tot een risicogroep en de overtuiging al tegen influenza beschermd te zijn door de vele patiëntcontacten. Ongeveer 4 op de 5 huisartsen adviseert influenzavaccinatie voor hun praktijkpersoneel. Een opvallende bevinding was dat een meerderheid van de huisartsen een placebogecontroleerde studie onder ouderen op de uitkomstmaten ziekenhuisopname en/of sterfte wenselijk vindt. Het positieve gedrag ten aanzien van influenzavaccinatie wordt weerspiegeld door de meerderheid van de huisartsen die een influenzavaccinatie neemt en influenzavaccinatie aanbeveelt aan hun praktijkpersoneel. Desondanks moet de houding van huisartsen ten aanzien van verplichtstelling van influenzavaccinatie en de gunstige effecten van influenzavaccinatie bij gezonde ouderen op ernstige morbiditeit en mortaliteit als aarzelend worden beschouwd.

Onderzoek doet vermoeden dat vaccinatie influenza-gerelateerde complicaties kan voorkomen of verminderen en een residuele bescherming kan bieden tegen influenzastammen die later kunnen circuleren. Daardoor zou influenzavaccinatie een effect op sterfte kunnen hebben dat langer duurt dan één influenzaseizoen. In hoofdstuk 4 evalueerden we de langetermijnsterfte onder ouderen, die in het seizoen 1991/1992 een eenmalige influenzavaccinatie ontvingen in het kader van een grote influenzatrial. We konden in een follow-up van 25 jaar geen overall verband aantonen tussen eenmalige influenzavaccinatie en langetermijnsterfte - hetzij de totale sterfte of de sterfte door specifieke doodsoorzaken. Ook een overall langetermijneffect van vaccinatie op seizoensgebonden sterfte konden we niet aantonen. De puntschatting van de overleving was echter consequent in het voordeel van de vaccingroep gedurende de gehele follow-up, maar bleek statistisch niet significant. Gezien de discussie over de invloed van leeftijd op de effectiviteit van vaccinatie, analyseerden we de sterftegegevens ook voor afzonderlijke leeftijdsgroepen. De analyse binnen de subgroep 60-64 jarigen suggereerde dat (eenmalige) vaccinatie een relevant langetermijneffect van 20 maanden kan hebben op de mediane overleving. Dit resultaat kan derhalve wel als een ondersteunende aanwijzing worden beschouwd, maar niet als sluitend bewijs. Rekening houdend met de beperkte power en met de bevindingen in de subgroep jongere ouderen, rechtvaardigen onze resultaten nader onderzoek naar het langetermijneffect van influenzavaccinatie op de mortaliteit bij ouderen. Onderzoekers die de effectiviteit van influenzavaccinatie - en mogelijk ook de meerwaarde van nieuwe vaccins - willen evalueren, doen er goed aan hun onderzoeksopzet geschikt te maken voor een langdurige follow-up.

Bovengenoemde onderzoeksgegevens boden niet alleen inzicht in de relatie tussen vaccinatie en sterftereductie, maar ook een unieke gelegenheid de immuunrespons die volgde op influenzavaccinatie in relatie tot overleving op de lange termijn te bestuderen. Dit onderzochten we in hoofdstuk 5. We maakten hierbij wederom gebruik van de placebo-gecontroleerde influenzavaccinatietrial uitgevoerd in 1991/1992 waarbij destijds 927 proefpersonen een influenzavaccinatie kregen toegediend en bij 905 van hen de immuunrespons voor alle 
influenzastammen die in het vaccin zaten was bepaald. Deze studie onder gevaccineerden kon geen relatie aantonen tussen de hoogte van de immuunrespons volgend op de influenzavaccinatie en overleving gedurende de periode van 25 jaar follow-up. Opvallend genoeg zagen we zelfs een omgekeerde relatie bij de jongere ouderen (60-64 jaar): hierbij was de sterfte binnen de groep met een krachtige immuunrespons op het vaccin groter. Mogelijk was deze bevinding vertekend door het rookgedrag van deze personen. In het verlengde van de hoofdbevindingen van onze studie, willen we benadrukken dat zelfs als er geen positieve relatie zou bestaan tussen de hoogte van de antilichaamrespons na influenzavaccinatie en overleving op lange termijn, dit niet betekent dat het functioneren van het immuunsysteem geen invloed heeft op de levensverwachting. Zoals eerder uitgelegd, was de immuunrespons die we hebben gemeten beperkt tot de respons op influenzavaccinatie en daarom mogelijk niet representatief voor de respons van het immuunsysteem in bredere zin.

Tijdens de influenzaseizoenen neemt de werkdruk van zorgpersoneel toe, evenals de bedbezetting en de opnameduur van patiënten. Dit kan leiden tot capaciteitsproblemen en obstructie van de zorgketen. Influenzavaccinatie speelt een belangrijke rol in het beperken van de gevolgen van influenza. Echter, ook goede diagnostiek naar influenza zou kunnen helpen om deze zorgvraag het hoofd te bieden. In hoofdstuk 6, bestudeerden we of sneltests - ook wel 'point-of-care'-tests genoemd - op influenza van meerwaarde zijn in de huisartsenpraktijk en op de Spoedeisende Hulp. Hiertoe bestudeerden we alle gerandomiseerde studies naar het effect van influenzasneltests op ten minste één van de klinisch relevante uitkomstmaten (gebruik van antibiotica en antivirale middelen, verblijfsduur op de Spoedeisende Hulp en opnameduur in het ziekenhuis). Uit deze studie kwam naar voren dat er meerdere sneltests beschikbaar zijn waarmee de diagnose 'influenza' binnen circa 30 minuten gesteld kan worden. Sneltests die gebruikmaken van antigeendetectie zijn makkelijk in gebruik, maar hebben een beperkte analytische accuratesse. Sneltests die gebruikmaken van de PCR-techniek zijn accuraat, maar behoeven directe ondersteuning van het medisch laboratorium; hierdoor zijn deze tests alleen inzetbaar in ziekenhuizen. Er is weinig direct bewijs voor de klinische effectiviteit van influenzasneltests op de Spoedeisende Hulp en bewijs voor de klinische effectiviteit van influenzasneltests in de huisartsenpraktijk ontbreekt. Influenzasneltests kunnen de ligduur op de Spoedeisende Hulp verminderen en de doorstroom van patiënten in het ziekenhuis verbeteren, mits capaciteitsproblemen dit niet belemmeren. De huidige literatuur rechtvaardigt de inzet van influenzasneltests die gebruikmaken van een PCR-techniek op de Spoedeisende Hulp. Het is vooralsnog een brug te ver om influenzasneltests ook in de huisartsenpraktijk te implementeren. 


\section{Eindconclusies}

De impact van influenza op het individu en de samenleving is aanzienlijk en kan deels worden voorkomen of verminderd door influenzavaccinatie, en point-of-care-testen in specifieke settings zoals spoedeisende hulpafdelingen. De huisarts speelt een belangrijke rol in de implementatie van influenzavaccinatie, en de houding en overtuigingen van de huisartsen met betrekking tot vaccinatie zijn belangrijk omdat deze kunnen dienen als promotor van het vaccinatiebeleid. Ten slotte heeft dit proefschrift het doel om de potentie van lange termijn follow-up van interventiestudies te benadrukken, alsook het belang te onderstrepen van ethische en methodologische aspecten van onderzoek dat betrekking heeft op evaluatie van de effecten van influenzavaccinatie. 

Addendum

Curriculum vitae 



\section{CURRICULUM VITAE}

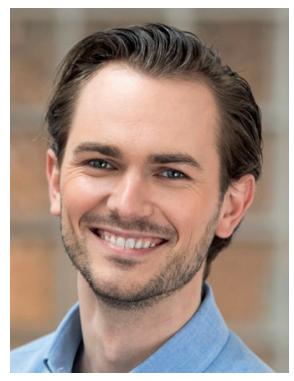

Ruud Verhees was born in Geldrop, the Netherland, on November 22, 1988. After high school graduation (cum laude) at Pleincollege Sint-Joris, Eindhoven in 2007, he moved to Maastricht and studied Medicine at the Faculty of Health Medicine and Lifesciences of Maastricht University. Several times he was awarded belonging to the 'Top 3\%' students of his academic year. After his clinical rotations (including rotations in Nepal and Uganda) he completed his Master's degree in 2014. He gained work experience at an Emergency Department and in 2015, he started his medical specialisation as a general practitioner in training at Maastricht University. During the first year of specialisation, Ruud began combining his traineeship with a PhD (AIOTHO-track) under supervision of Prof. dr. G.J. Dinant, Prof. dr. J.A. Knottnerus and Dr. C.T. Thijs. During his PhD he obtained the Kootstra Talent Fellowship and during the last year of his traineeship, he successfully applied for the Hippocrates Program (Wonca Europe, Finland). He completed his medical specialisation in 2019 and was awarded by the Dutch training centre for family physicians (Huisartsopleiding Nederland) for his exceptional high level of knowledge. Ruud currently lives in Eindhoven, works as a family physician in a large urban general practice and assesses the communicational skills of general practitioners in training at Maastricht University. 

Addendum

List of publications 



\section{LIST OF PUBLICATIONS}

1. Verhees RAF, Snellings R, Dinant GJ, Knottnerus JA. Influenza vaccination among Dutch general practitioners and their attitude toward influenza vaccination in the elderly. Hum Vaccin Immunother. 2020;16:2709-18.

2. Verhees RA, Thijs C, Ambergen T, Dinant GJ, Knottnerus JA. Griepvaccinatie en langetermijnsterfte. Follow-up 25 jaar na een RCT bij ouderen. Huisarts Wet 2020;63:16-9.

3. Verhees RAF, Lutgens S, Kusters R, Dinant GJ, Cals J. Influenzasneltests voor de huisartsenpraktijk en SEH. Ned Tijdschr Geneeskd. 2019;163:D3806.

4. Verhees RAF, Maak kennis met. Ned Tijdschr Geneeskd. 2019;163:B1616.

5. Verhees RAF, Thijs C, Ambergen T, Dinant GJ, Knottnerus JA. Influenza vaccination in the elderly: 25 years follow-up of a randomized controlled trial. No impact on long-term mortality. PLoS One. 2019;14:e0216983.

6. Verhees RAF, Dondorp W, Thijs C, Dinant GJ, Knottnerus A. Effect influenzavaccinatie op sterfte onder ouderen. Ned Tijdschr Geneeskd. 2018;162:D3177.

7. Verhees RAF, Dondorp W, Thijs C, Dinant GJ, Knottnerus JA. Influenza vaccination in the elderly: Is a trial on mortality ethically acceptable? Vaccine. 2018;36:2991-7.

8. Verhees R, Knottnerus JA. Griepvaccinatie: bewijs voor bescherming, weinig studies van goede kwaliteit. Huisarts Wet.2018;61(9):62-3.

9. Verhees R. Influenzavaccinatie voorkomt meer dan een griepje. Huisarts Wet.2017;60(12):667.

10. Verhees R, Muris J. Pneumokokkenvaccinatie bij patiënten met COPD. Huisarts Wet. 2017;60(10):534.

11. Verhees RA, Besselaar AT, van Aken MH, Jansen FH, Pelleboer RA. Van plexuslaesie naar supracondylaire humerusfractuur. Ned Tijdschr Geneeskd. 2015;160:35-8.

12. Verhees RA, Pelleboer R, Jansen F. Een neonat met cysten in een cyste. Med Contact. 2015;70:1685.

13. Verhees RA. Effectiviteit van lasertherapie bij onychomycose. Huisarts Wet. 2016;59:140.

14. Verhees RA, Nanlohy Manuhutu M. Hematurie en een hypertympane zwelling. Med Contact. 2016;71:25. 

Addendum

Dankwoord 



\section{DANKWOORD}

In dit laatste, meest persoonlijke deel van het proefschrift, wil ik graag stilstaan bij alle mensen die een rol hebben gespeeld in het tot stand komen van dit proefschrift. Soms was deze onmisbaar groot, andere keren bescheiden en subtiel. Wat deze rol ook exact is geweest, voor mij is de bijdrage van deze mensen van grote betekenis geweest. Vandaar dat ik een aantal van hen graag expliciet wil benoemen en bedanken.

Allereerst mijn promotieteam: mijn promotoren Geert-Jan en André en copromotor Carel.

Geert-Jan. Omdat wij beiden door onze collega's als praatgraag gezien worden, zal ik proberen de word-count binnen de perken te houden en een mooie summary te schrijven die alsnog recht doet aan de periode waarin we hebben samengewerkt. Was het toch een manuscript geworden, zouden er ongetwijfeld leuke koppen te bedenken zijn die de suggestie van een roman zouden wekken. Ze zouden onder andere betrekking hebben op onze sparsessies op de vakgroep, de etentjes bij Gerda en jou (al dan niet met slak op de schouder), de klopjacht op de geelbuikvuurpad, afdalingen in jullie mergelgroeve en de koffiepauzes in de keuken op Deb of bij Marc op de kamer. Deze momenten geven weer op wat voor een bijzondere manier wij gedurende mijn promotie met elkaar om zijn gegaan. Het enthousiasme waarmee je mij als promovendus hebt ontvangen en hebt begeleid is afgelopen zes jaar alleen maar groter geworden. Te allen tijde ben jij er als promotor, maar ook als 'promotie-coach' er voor me geweest. Ik betrap me erop dat ik met name de laatste jaren het woord 'vriendschap' steeds vaker heb gekozen om onze relatie te beschrijven. Dit omdat je nooit boven, maar altijd naast of zelfs achter me hebt gestaan. Dank voor je vertrouwen in mij, je optimisme, je brede zienswijze, geduld, humor, je bereidwilligheid me met alles en iedereen in contact te brengen en je blijk van trots wanneer ik namens ons team publiceerde. Bedankt ook voor je openheid, je benaderbaarheid en niet in de laatste plaats de brainstorm sessies die we samen hebben gehad, letterlijk en figuurlijk.

André. Ik heb vele mensen in het verleden al hun bewondering voor jou horen uitspreken. Bij deze wil ik dat op mijn eigen manier doen. Direct vraag ik me af wat de bewijskracht van iets is, als meerdere mensen het onafhankelijk van elkaar uitspreken over één persoon. Ik durf te stellen dat het meer dan louter empirisch bewijs is! Ik dwaal af. Voordat ik je ontmoette, kende ik je wapenfeiten al een beetje. Ik kan nu, zes jaar later, best eerlijk zeggen dat ik een beetje nerveus was toen Geert-Jan me bij jou als academisch zwaargewicht in de promotie-arena gooide. Gelukkig is dat gevoel snel gezakt. Wat onverminderd aanwezig bleef was mijn bewondering voor jou als wetenschapper en als mens. Als jij bij onze vergaderingen aanwezig was, wist ik dat het proces goed bewaakt werd en alles wat we zouden moeten bespreken ook daadwerkelijk op tafel kwam. Met onvermoeibare doortastendheid zat jij er altijd bovenop en ontsnapte niets aan je aandacht. Ik ben me blijven verbazen over jouw scherpzinnigheid, hoe je mijn manuscripten weer van goed commentaar wist te voorzien maar vooral ook de nuances wist aan te brengen waardoor onze teksten ineens weer klopten. Ik heb wel eens gedacht 'moet André anders niet mijn thesis schrijven, 
hij kan dit vast beter!' Toch liet je mij altijd de ruimte om mijn eigen pad te bewandelen. Het ethiek stuk, het POCT artikel, je ontving al mijn ideeën met enthousiasme maar ook met realisme en een kritische blik om zo de 'koekoekseieren' uit ons nest te houden. Ik zou je tekort doen als ik alleen bij je wetenschappelijke kwaliteiten zou stilstaan. Je kwam altijd goed gemutst met je mobiele Watson op wieltjes achter je aangetrokken, de vakgroep opgelopen. Dank voor je (soms droge) humor waar altijd meer gelaagdheid in aan was gebracht dan 'a priori' vermoed werd. Tot slot dank voor jouw geweldige feesten; je 'afscheid' van de UM op chateau Sint Gerlach en feest in de Bonbonnière. Dit gaf een uitzonderlijke inkijk in de dansstijl van hoogleraren die me ook lang zal bijblijven.

Carel. De analyseplannen of methodologische dilemma's waar André me soms voor plaatste, kon ik vervolgens samen met jou proberen te kraken. Dank voor jouw geduld en (om in de termen van het Centraal Bureau voor de Statistiek te spreken) het letterlijk over de schouder meekijken. Het laatste hoofdstuk bleek een serieuze kluif te zijn, de term 'anafylaxie' zou bijna een eufemisme zijn. Doordat je dit goed aanvoelde konden we samen, als het kleine blauwe naaldje dat de Musculus Deltoideus van de 60-plusser aanprikt, door de materie gaan. Bedankt voor jouw zorgvuldige commentaar. Ik zag er vaak tegenop, juist omdat ik wist dat het betekenisvol en waardevol zou zijn, en daarmee onherroepelijk tot aanpassingen van het manuscript zou leiden. De stukken werden er echter altijd beter op en zonder jou zou ons team niet compleet zijn geweest. Ook in de voorbereidingen voor de promotie bleek je een soort extern logboek voor me waarbij je altijd weer wist te reconstrueren waarom we destijds bepaalde keuzes hadden gemaakt. Dankje Carel.

Walter. Bedankt voor jouw fijne samenwerking en geduld als co-auteur van mijn laatste inhoudelijke hoofdstuk. We hebben elkaar een aantal keer getroffen aan het Ei in Amsterdam. Bij jou kon ik even te rade gaan als de virologie me toch te complex werd. Je wist me dan als een volleerd docent tot op de punt, komma en het hemagglutinine eiwit uit te leggen hoe het ook alweer in elkaar stak. Zoals ik eerder al zei, laten we snel samen met een goed bier (dat mag een Duits bier zijn) proosten op deze bekroning van dit werk.

Suzanne. Ook jou wil ik bedanken voor je input als medisch microbioloog in het tot stand komen van een van onze artikelen. Ik kon je altijd mailen met vragen en zelfs toen ik's ochtends vroeg een interview voor BNR nieuwsradio moest geven, kon ik de avond van tevoren vanuit een wijnkabinet in Duitsland toch nog even alles met je doorlopen en fact-checken. Dankjewel!

Leden van de beoordelingscommissie. Ook jullie wil ik danken voor jullie tijd, bereidwilligheid en energie om mijn werk te lezen en te beoordelen.

Dan mijn lieve vrienden. Hoe fijn is het te kunnen zeggen blij voor en met elkaar te zijn, trots op elkaar te zijn. Daarom wil ik graag proberen in woorden te vangen wat een aantal van deze bijzondere mensen gedurende de afgelopen jaren voor mij en mijn promotie betekend heeft.

Eric, boyke! Wat hebben we in Maastricht een geweldige tijd samen beleefd. Het was voor mij dan ook direct duidelijk dat ik jou tijdens mijn verdediging aan m'n zijde wilde hebben. Voor mijn gevoel hebben we echt een vriendschap 2.0 kunnen opbouwen tijdens onze PhD, waarin we de 
perfecte cocktail van werk en ontspanning als adjuvans hebben gevonden. We zagen elkaar veel en als het even kon sportten we tijdens onze PhD dagen in de middag bij UM sport. Onze sets van 8-6-4 muscle ups zijn publicatiewaardig, dus bij deze verschijnen ze in de wetenschappelijke literatuur! Ons sportieve hoogtepunt is naar mijn idee altijd nog de zelfgeorganiseerde bootcamp op de Sint Pieter toen het natuurbad geopend werd. Ik heb zo genoten van deze tijd. Pingpongen in het Student Hotel waarbij we de ballen 't hele hotel door sloegen, ons spontane strandavontuur met Wim op een 'vrije dag', mosselen aan het zwembad en zeer recent nog onze wielrenvakantie op Mallorca waar jij je weer ontpopte tot een echte KOM-tador. Soms topten we de academische dag nog even af met een lezing van Studium Generale over het heelal, vrije wil (hoe bizar interessant was die lezing) of de evolutietheorie. Onze PhD lijkt daarmee een van de rode draden die door onze vriendschap gewoven is. Ik weet soms niet meer goed of we nu gewoon vrij hadden, of eigenlijk iets voor ons onderzoek aan het doen waren of hadden moeten doen. Wat dat betreft was het onderzoek dat we deden 'never ending' en die toewijding voelden we allebei sterk. We konden beiden uitspreken er soms echt helemaal klaar mee te zijn. Ik heb de stelling 'research is a bitch' E.L.A. Fonseca Wald, toch maar niet in dit proefschrift opgenomen. Dank voor jouw motiverende woorden, het luisteren, oprechte interesse in het onderzoek en in mij als vriend. Ik kon je over alles vragen mee te denken want je interesse was groot en je was overal ook wel een beetje expert in. Mede dankzij jou en Berrie heb ik met succes de Kootstra Beurs kunnen binnenhalen, een springplank voor mijn PhD. Je bent er altijd voor me geweest, ook toen ik even nergens anders heen kon. Ik kijk er echt naar uit het leven met je te blijven onderzoeken!

Berrie. Ook jij bent zo'n trouwe rakker en maakt deel uit van mijn 'et al'. Ook wij gaan inmiddels way back en klikten de eerste weken van de geneeskunde opleiding al tijdens een bijeenkomst waarbij we het Probleem Gestuurd Onderwijs eens goed onder de loep namen. Feit dat we hier allebei zaten zegt heel veel over ons: kritisch en onderzoekend. Dit blijkt ook uit de Top 3\% wedloop die we samen met Tom hielden. Wat zijn we eigenlijk ook een strevers (geweest) he! Gelukkig weten we heel goed wat er nog meer belangrijk is in het leven. We hebben een mooie vriendschap opgebouwd. Jij maakte ons sporttrio met Eric compleet en bracht ons beide naar een ander nivo. Dat heb je ook binnen mijn onderzoek gedaan. Jouw hulp en gekrabbel bij de beursaanvragen die ik maakte vergeet ik niet, en ik weet ook zeker dat jouw inside information me geen windeieren heeft gelegd. Wat is het jammer dat we deze super huisarts aan ons buurland België zijn verloren. Als ik je praktijkperikelen goed beluister, zoek ik maar troost in het feit dat ze je daar wellicht harder nodig hebben dan in Nederland! Ik zou je in ieder geval mijn gezondheid hebben toevertrouwd. Bedankt voor jouw telefoontjes en betrokkenheid. Je bent een gouden jongen.

Gregster. Wat hebben ook wij een mooie tocht afgelegd. Vanaf de eerste onderwijsgroep was het gelijk raak. Wat was je verdwaald in het begin kerel, een hotelkamer op Randwyck, helemaal uit het hoge Noorden, volgens mij verstond je me in het begin ook nog maar amper! Ik weet nog dat je me veel later vertelde dat je blij was dat ik je aansprak en dat we zo makkelijk aansluiting vonden. Wat hebben we veel heerlijke avonden in de Tischbeinstraat aan jouw houten tafel doorgebracht. 
Jij kookte voortreffelijk en we trokken er de hele avond voor uit. Soms sloten Milou en Tina aan, wat onze dynamieken altijd op scherp stelde. Wat waren we toch een stel knappe jongens, niet? Over geneeskunde spraken we eigenlijk heel weinig. Waar we 't wel over hadden, mag tijdens de borrel aan ons gevraagd worden. Ik bewonder jouw arbeidsethos. Je ging voor je opleiding naar Duitsland terwijl jouw Duits nog slechter was dan je Brabantse of Limburgse imitaties, en combineerde dat vervolgens ook nog eens met een PhD die je zelf grotendeels aan elkaar knoopte. Ik ben echt blij voor je dat ook jij de eindstreep nu aan het halen bent: je bent net klaar als chirurg en ook jij rond je PhD nu af. Het vooruitzicht op je fellowship in Rotterdam is serieus bonkers. Richt je alvast een kamertje voor me in voor in de weekenden?

Mervyn, Judith en kleine Oliver. Ook jullie wil ik bedanken in mijn boekje. Bedankt Mervyn voor de vriend die je al meer dan 30 jaar voor me bent. Onze vriendschap heeft afgelopen jaar nog een extra boost gekregen doordat jullie mij een soort tweede thuis tijdens de coronacrisis hebben geboden. Tegen anderen had ik het over mijn 'Foster Parents' gezinnetje. Indirect heeft dat heel positief bijgedragen aan de afronding van mijn proefschrift. Judith, ook jij bedankt voor je omarming en jouw close reading toen mijn proefschrift af was. Oliver, je kunt nu nog niet lezen, maar gefeliciteerd met je eerste vermelding in de wetenschappelijke literatuur kereltje. Gevoelsmatig lever jij over pak 25 jaar ook je proefschrift in. Als ik je daar nog eens bij kan helpen, let me know!

Lennart. Geert-Jan (onze promotor) is een goede netwerker en heeft intuïtief haarfijn aangevoeld dat wij wel eens goed met elkaar zouden kunnen opschieten. Zo goed zelfs, dat jij net als Eric mijn paranymf bent. Ik weet nog dat Geert-Jan ons aan elkaar voorstelde en dat ik je tijdens ons terrasje op UNS 30 een beetje wegwijs makkte op de vakgroep. Wat jammer eigenlijk dat we elkaar niet eerder zijn tegengekomen, Dommelen en Leende, het had zo gekund. Ik leerde je in het begin als collega kennen, maar gaandeweg gingen we meer en meer met elkaar om. Het wielrennen was een mooi smeermiddel om ons ook op vriendschappelijk nivo dichter bij elkaar te brengen. Puur op karakter pers jij er ongetraind even 100 km door het heuvelland uit, zo sterk ben je. Dat typeert jou als sportmaatje, maar ook als collega en vriend. Wat hebben we een leuke tijd in Engeland gehad, toen ik je kwam opzoeken tijdens je hospice stage. Onwerkelijk tevens, hoe wij in Londen aan de bar onze Goose Islands dronken, terwijl de rest van Europa op dat moment de deuren sloot door de coronacrisis. Ook mijn laatste chapter kwam in die periode in een soort lock-down terecht. Jij hebt dat gezien en keer op keer meegedacht over hoe ik het laatste hoofdstuk kon afronden. Bedankt voor de hulp die je me aangeboden hebt. Ik hoop dat we nog veel ritten samen gaan maken en leuke dagen met het peloton beleven. Wie weet gaan we nog een mooie onderzoekssamenwerking in de toekomst tegemoet. Maar... eerst maar eens lekker wat uren (en euro's) in de praktijk maken!

Maastricht Boys, haha, hadden we echt niet een andere naam voor onze groep kunnen bedenken?! Bedankt voor jullie vriendschap en de leuke mannenweekenden. Ten tijde van dit schrijven kan ik zeggen dat het mannenweekend voor volgende week nog steeds niet gepland is, maar bij deze weten jullie waar de tijd wél in heeft gezeten. Maastricht is en blijft wel onze 
home-town waar we allemaal mooie herinneringen aan hebben, niet in de laatste plaats omdat we elkaar hier hebben leren kennen. Jullie hebben van deze stad een thuis voor me gemaakt, waarvoor bedankt! Bij deze wil ik ook alle andere lieve vrienden en vriendinnen waarmee ik stuk voor stuk bijzondere banden heb opgebouwd bedanken voor hun vriendschap. Myreen, dankje voor alle onvoorwaardelijke steun die ik in het verleden heb mogen ontvangen. Ik weet nu nog beter hoe waardevol dit is.

Dan, Team BBQ/kapsalon/PhD. Het begon met een BBQ bij Jetse in de tuin. Allemaal klaar met de geneeskunde opleiding en stuk voor stuk ambities te gaan promoveren. Een groepsapp werd snel gemaakt en het was duidelijk dat hier de eerder genoemde Mesh-termen niet in mochten ontbreken. Over onderzoek hebben we het inhoudelijk nooit gehad. Immers was dit het groepje 'snijders', legde ik aan anderen altijd uit. Overdags waren jullie patiënten de pineut, en als we elkaar 's avonds zagen, ging de botte bijl menig keer door een ieder van ons. Soms zelfs letterlijk, zoals in Egmond aan Zee! Wat hebben we veel gelachen! Tim, toch wel de grootste blaaskaak, maar tegelijkertijd de liefste van ons allemaal. Jetse, jij en ik sloten onze vriendschap tijdens jouw keuzestage chirurgie. Kan het zijn dat wij dit coschap zo leuk hebben gemaakt dat je daardoor in opleiding tot chirurg bent gegaan? Jij promoveerde als eerste van ons. Een geweldige dag met kastelen, bubbels, een lijpe verdediging en leuk feest. Ik stond daar aan je zijde als paranymf, een herinnering die ik nog altijd koester. Jouw openheid en oprechtheid worden niet alleen door mij, maar ook op je werk gewaardeerd en dit maakt jou tot een bijzonder chirurg. Er zijn momenten dat we allebei even verifïren dat we er zijn voor elkaar, zoals recent nog met de waterramp in Limburg. Wat ben ik blij dat dit goed is afgelopen, en wat bewonder ik je voor jouw optimisme hierin. Jetse, het duurde wel een paar jaar voordat ik aan je gewend was, maar inmiddels kunnen we lezen en schrijven met elkaar. Helder en direct, geen blad voor de mond. Een onwijs pientere orthopeed in opleiding. Net niet pienter genoeg, want ik leverde mijn proefschrift enkele weken vóór jou in! Ook wij maakten mooie ritten, soms samen, soms met Tom Testosteron. Ondanks al het sulfaat in die kapsalons die jij met verve voor ons bereidde, is ons testosteron gehalte altijd nog niet gedaald. Laten we vooral doorgaan met dit mooie clubje en hopen dat we dit spoedig mogen omdopen tot Team BBQ/kapsalon/post-PhD! Tom, net als Tim en Jetse, bewonder ik ook jou. Top clinicus (de enige uroloog in Nederland die direct een baan vond?), top academicus en top sporter. Drie keer fietsen en je bent weer in bloedvorm waarbij Jetse en ik alleen nog maar hijgend in je racewiel kunnen blijven hangen. Ook jij ziet in dat het leven meer is dan een race en ik hoop dat we deze balans goed blijven bewaken in onze levens.

Vakgroep Maastricht. Ik twijfel even of ik eerst de vakgroepvoorzitter prof. Muris moet noemen, of toch de Bonny and Clide van onze vakgroep; de secretaresses Ine en Ellen. Vooruit, Ine en Ellen, om te beginnen met Ine. Ik was nog geen week verbonden aan de vakgroep of we lagen al in elkaars vaarwater. Zomaar schuiven met bureaus was 'not done', daar moest eerst permissie voor komen en wel van de hoogste orde, mw. Siegelaer. Zoals een promotietraject een leercurve kent, kende ook onze sociale interactie deze. Als ik de kromming moet omschrijven zou deze eerst 
heel vlak oplopen (vals plat voor een wielrenner) en daarna een prachtig stijgingspercentage à la Cauberg volgen. Dank voor de leuke tijd en het karakter wat je aan de vakgroep hebt gegeven. Ellen, ook jij dank voor de gezellige praatjes in de gang of op de kamer.

Jean. Het grootste deel van mijn promotietijd stond jij aan het roer van de vakgroep. Het spreekt voor zich dat ik ook jou wil danken voor de mogelijkheden die de vakgroep me heeft geboden.

Karin. Lange tijd ben ik jouw kamergenoot geweest. Je moet je wel een beetje met mij opgescheept hebben gevoeld; jij als ervaren onderzoeksassistent, ik als de Benjamin die continu de deur uit vloog voor koffie hier, een lunch daar of om te sporten. Dankje voor jouw hulp bij het reconstrueren van de dataset die een belangrijke basis was voor mijn proefschrift!

Bij deze wil ik ook mijn dank uitspreken voor Phile Govaert, de grondlegger van de influenzatrial uitgevoerd in Maastricht waar ik in mijn artikelen herhaaldelijk naar verwijs en mijn vervolgstudie op heb gebaseerd. Phile is helaas in 2021 overleden. Ik koester de herinnering aan het bezoek dat mijn promotoren en ik aflegden in zijn prachtige appartement aan het Charles Eyckpark in Maastricht. Zijn opmerking ten aanzien van de vaccinatieweerstand bij hem in de huisartsenpraktijk blijft me goed bij en inspireerde me zelfs voor een van de stellingen in dit proefschrift: 'Als je vreest zo beroerd te worden van een griepspuit, bedenk dan hoe ziek je door de griep zou kunnen worden.'

Jochen en Marc. Voor mijn gevoel toch nog altijd meer de junioren dan senioren op de vakgroep. Jochen, ik kan naar een officiële publicatie verwijzen waarin ik mijn bewondering voor je al uitgesproken heb: Ned Tijdschr Geneeskd. 2019;163:B1616. Dank, niet alleen voor jouw inspanningen als co-auteur binnen ons POCT stuk, maar vooral voor de lach die we vaak bij elkaar op het gezicht wisten te toveren. Ik hoefde mijn hoofd maar voorbij je kamerdeur te steken of we begonnen allebei te grijnzen. Marc, wat jammer dat we elkaar tijdens de coronacrisis nagenoeg niet meer hebben gezien. Dankje voor de koffiemomentjes en belangstelling. Ik denk dat het hoog tijd wordt dat we samen met Jochen nog eens een 'fries-at-war' eten en houd me aanbevolen voor jouw Orka-trips in Noorwegen.

Pascal. Bijna voordat ik mijn mail aan je gestuurd had, ontving ik al antwoord van je. Bedankt voor jouw super snelle ICT-service en de gezellige koffies in de pauze.

De junior (en semi-junior) collega's van de vakgroep. Hier ga ik vast wat namen vergeten dus is dit stukje aan jullie allen gericht. Een aantal wil ik er in het bijzonder noemen: Krista, Jolijn, Eefje, Maartje, Anouk, Floor, Marjolein, Angel, Raissa, Stijn en Michelle. Wat heb ik een leuke tijd met jullie gehad op de vakgroep. Ik heb maar 1 jaar fulltime onderzoek gedaan, maar hierdoor hebben we een goede basis kunnen leggen waardoor het altijd weer leuk was even bij jullie binnen te wippen als ik toevallig voor onderwijs beneden of voor een overleg met mijn promotieteam op de gang was. De 'Heel Huisartsgeneeskunde Bakt' momenten (meesterwerk of misbaksel) waren wel een absoluut hoogtepunt van de week. Ook wil ik het trio aan het eind van de gang, Annerika, Esther en Anneke, bedanken voor alle gezellige praatjes. 
Dan mijn praktijkopleiders, Irene en Frans, John en Tom. Ik bedenk me nu pas dat ik het voorrecht heb gehad om maar liefs vier opleiders te hebben, daar waar twee de norm is. Van jullie allen heb ik veel geleerd en mede door jullie ben ik de huisarts geworden die ik nu ben. Wat hebben we een prachtige dag gehad toen ik mijn huisartsendiploma uitgereikt kreeg. Dank voor jullie coaching maar ook de ruimte die en het begrip dat ik heb gekregen voor mijn promotieonderzoek. Frans en John, jullie wil ik expliciet bedanken voor de vriendschap die we hebben opgebouwd na mijn opleidingstijd bij jullie in de praktijk. Felix, ook jou wil ik hier kort noemen als blijk van dank voor je wijsheden. Dorrit, dank voor de 'intervisie' en levenslessen.

De welpjes. Het is inmiddels een cliché geworden dat je een bijzondere band opbouwt en behoudt met de huisartsen-in-opleiding die je in je eerste jaar treft. En toch is het waar. We hebben een ontzettend leuke opleidingstijd gedeeld, waarin we altijd een veilig klimaat wisten te creëren om alles (en soms ook net iets teveel) met elkaar te delen. We hebben echt veel lol gehad maar ook de verbinding met elkaar kunnen vinden, en ik denk dat dat ons als huisartsen, maar ook onze beroepsgroep typeert. Als ik gepromoveerd ben houden jullie een lesje EBM van me tegoed...of nee, toch maar liever een biertje terwijl we onze praktijkperikelen hopelijk gauw weer in een gezellig restaurantje bespreken.

Huisartsopleiding Maastricht, Joost, Bas, de dames op Deb en medewerkers van de $\mathrm{SBOH}$. Volgens mij kende zowat de hele huisartsopleiding mijn naam omdat ik ook zo'n 'lastige' AIOTHO was, toch liever het $3^{\mathrm{e}}$ jaar van de opleiding in Eindhoven wilde volgen en dan ook nog eens graag een paar weken naar Finland wilde voor het Hippocrates programma. Daar waar jullie konden hebben jullie mijn gehele opleiding op maat gemaakt. Dit maatpak heeft heerlijk gezeten, waar ik jullie hartelijk voor wil danken. Ik kijk nu videotoetsen voor de opleiding na, waardoor ik me altijd nog wat met jullie verbonden voel. Hopelijk heb ik later ook de ruimte om ook een docentfunctie te kunnen vervullen waardoor ik toch een beetje met jullie in contact kan blijven.

Team van huisartsenpraktijk SGE Stratum, Eindhoven. Ook jullie wil ik graag noemen in mijn dankwoord. Hoewel jullie geen actieve bijdrage hebben geleverd aan het tot stand komen van het proefschrift zijn jullie wel belangrijk voor me geweest in de afrondende periode. De laatste fase van mijn proefschrift was een bewogen tijd waarin veel is gebeurd. In meerdere levensdomeinen is toen veel veranderd; privé, wonen, werk. Mijn waarneemperiode in Geldrop liep precies ten einde toen Teike me in contact bracht met Marleen om te zien of er interesse was om 'tijdelijk' in Stratum te komen werken. Inmiddels werk ik er al weer 1.5 jaar! Dankzij alle mango-momentjes waarin jullie blijk van waardering en collegialiteit gaven, heb ik me als 'de waarnemer' heel erg onderdeel van het team gevoeld. Marleen, ik wil jou graag expliciet benoemen aangezien jij in de roerige tijden die de praktijk gekend heeft, het altijd voor iedereen goed hebt willen doen en hebt gedaan. Dankje dat jullie zo'n fijne collega's zijn. Misschien moeten we die extra vleugel toch maar gaan aanbouwen! 
Tot slot. Lieve pap, mam en Nick. Zonder de ruimte die jullie me lieten om te studeren, mijn eigen pad te bewandelen en vertrouwen te uiten in mijn kunnen, was ik niet de persoon geworden die ik nu ben. Dank jullie voor wat jullie voor me zijn en altijd zullen blijven: mijn familie. 